2016-09-01

\title{
Haptic Shape-Based Management of Robot Teams in Cordon and Patrol
}

Samuel Jacob McDonald

Brigham Young University

Follow this and additional works at: https://scholarsarchive.byu.edu/etd

Part of the Mechanical Engineering Commons

\section{BYU ScholarsArchive Citation}

McDonald, Samuel Jacob, "Haptic Shape-Based Management of Robot Teams in Cordon and Patrol" (2016). Theses and Dissertations. 6100.

https://scholarsarchive.byu.edu/etd/6100

This Thesis is brought to you for free and open access by BYU ScholarsArchive. It has been accepted for inclusion in Theses and Dissertations by an authorized administrator of BYU ScholarsArchive. For more information, please contact scholarsarchive@byu.edu, ellen_amatangelo@byu.edu. 
Haptic Shape-Based Management of Robot Teams in Cordon and Patrol

\title{
Samuel Jacob McDonald
}

\author{
A thesis submitted to the faculty of \\ Brigham Young University \\ in partial fulfillment of the requirements for the degree of \\ Master of Science
}

Mark B. Colton, Chair

Michael A. Goodrich

Timothy W. McLain

Department of Mechanical Engineering

Brigham Young University

2016

Copyright $\odot 2016$ Samuel Jacob McDonald

All Rights Reserved 


\author{
ABSTRACT \\ Haptic Shape-Based Management of Robot Teams in Cordon and Patrol \\ Samuel Jacob McDonald \\ Department of Mechanical Engineering, BYU \\ Master of Science
}

There are many current and future scenarios that require teams of air, ground or humanoid robots to gather information in complex and often dangerous environments, where it would be unreasonable or impossible for humans to be physically present [1-6]. The current state of the art involves a single robot being monitored by one or many human operators [7], but a single operator managing a team of autonomous robots is preferred as long as effective and time-efficient management of the team is maintained $[8,9]$. This is limited by the operator's ability to command actions of multiple robots, be aware of robot states, and respond to less important tasks, while accomplishing a primary objective defined by the application scenario. The operator's ability to multi-task could be improved with the use of a multimodal interface, using both visual and haptic feedback. This thesis investigates the use of haptic feedback in developing intuitive, shape-based interaction to maintain heads-up control and increase an operator's situation awareness (SA) while managing a robot team.

In this work, the autonomous behavior of the team is modeled after a patrol and cordon scenario, where the team travels to and surrounds buildings of interest. A novel approach that involves treating the team as a moldable volume is presented, where deformations of this volume correspond to changes in team shape. During surround mode, the operator may explore or manipulate the team shape to create custom formations around a building. A spacing interaction method also allows the operator to adjust how robots are spaced within the current shape. Separate haptic feedback is developed for each method to allow the operator to "feel" the shape or spacing manipulation. During travel mode, the operator chooses desired travel locations and receives feedback to help identify how and where the team travels.

RoTHSim, an experimental robot team haptic simulator, was developed and used as a test bed for single-operator management of a robot team in a multitasking reconnaissance and surveillance scenario. Using RoTHSim, a human subject experiment was conducted with 19 subjects to determine the effects of haptic feedback and task demand difficulty on levels of performance, SA and workload. Results from the experiment suggest that haptic feedback significantly improves operator performance in a reconnaissance task when task demand is higher, but may slightly increase operator workload. Due to the experimental setup, these results suggest that haptic feedback may make it easier for the operator to experience heads-up control of a team of autonomous robots. There were no significance differences on SA scores due to haptic feedback in this study.

Keywords: human-swarm interaction, haptic feedback, situation awareness 


\section{ACKNOWLEDGMENTS}

There are many people to thank for their help and support as I have worked to complete this thesis and my masters degree. I am very grateful to family, friends, professors and colleagues for their contributions to my success up to this point.

Firstly, I would like to thank my beloved wife Melissa and son Bennett for uplifting me in the hard times and celebrating with me in times of success. I would not be where I am today with the support of my family.

I need to thank Dr. Colton, my graduate advisor, for his trust in me in taking on a project that is outside of both our comfort zones. His knowledge, support and patience have allowed me to grow academically and personally more than I had ever hoped. I would also like to thank Dr. Goodrich for taking the time to teach me how to properly conduct a user study and always provide bright ideas when they are needed most. I thank my colleague, Kris Alder, for developing the swarm behavior that made this research possible.

This work was funded by the United States Army Research Laboratory through contract W911NF-14-1-0633. 


\section{TABLE OF CONTENTS}

LIST OF TABLES $\ldots \ldots \ldots \ldots \ldots \ldots \ldots \ldots \ldots \ldots \ldots$ vii

LIST OF FIGURES $\ldots \ldots \ldots \ldots \ldots \ldots \ldots \ldots$ viii

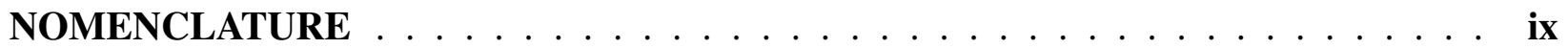

Chapter 1 Introduction . . . . . . . . . . . . . . . . . 1

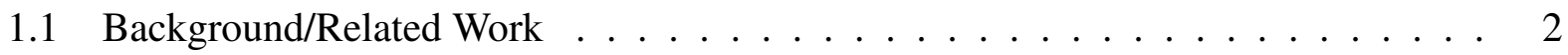

1.1.1 Swarm Robotics . . . . . . . . . . . . . . . . . . . 2

1.1 .2 Haptic Feedback . . . . . . . . . . . . . . . . . . . 3

1.1.3 Situation Awareness . . . . . . . . . . . . . . . . . 4

1.1.4 Workload ...................... 5

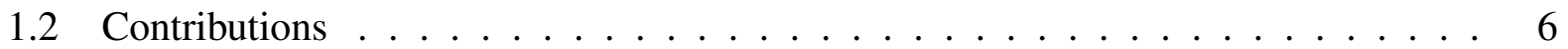

1.3 Thesis Overview . . . . . . . . . . . . . . . . . . 7

Chapter 2 Team Interaction and Haptic Feedback Development . . . . . . . . . . . 8

2.1 Robot Behaviors Model . . . . . . . . . . . . . . . . . . . . . . . . 8

2.1 .1 Team Modes . . . . . . . . . . . . . . . . . . . . . . . 9

2.1.2 Team Information and Control . . . . . . . . . . . . . . . . 10

2.2 Modeling Clay: A Haptic Metaphor . . . . . . . . . . . . . . . . . 10

2.2.1 Modes of Interaction . . . . . . . . . . . . . . . . . . . . 11

2.3 Ground Force Feedback . . . . . . . . . . . . . . . . . . . . . . . . . 12

2.4 Force Node Network . . . . . . . . . . . . . . . . . . . . . . . . . 13

2.5 Surround Interaction $\ldots \ldots \ldots \ldots$

2.5.1 Shape Exploration . . . . . . . . . . . . . . . . . . . . . 14

2.5.2 Shape Manipulation . . . . . . . . . . . . . . . . . . 15

2.5.3 Spacing Manipulation . . . . . . . . . . . . . . . 16

2.6 Travel Interaction . . . . . . . . . . . . . . . . . . . . . . . . . . . . . . . . . 19

2.6.1 Relative Travel . . . . . . . . . . . . . . . . . . . . . . . . . . . . . 19

2.6 .2 Shape Exploration . . . . . . . . . . . . . . . . . . 20

2.7 Chapter Summary . . . . . . . . . . . . . . . . . . . . . . . . . . 22

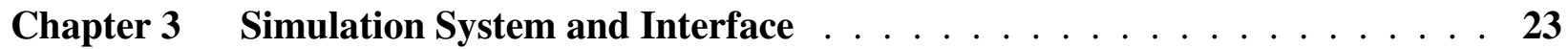

3.1 System Overview . . . . . . . . . . . . . . . . . . . . . . . 23

3.2 Novint Falcon and CHAI 3D . . . . . . . . . . . . . . . . . . . . . . 24

3.3 Robot Behaviors Server . . . . . . . . . . . . . . . . . . . . . . . . . 25

3.4 RoTHSim C++ Application . . . . . . . . . . . . . . . . . . . . . 26

3.4 .1 Team Display . . . . . . . . . . . . . . . . . . . . . . 26

3.4.2 Hotspots Implementation . . . . . . . . . . . . . . . . . . . . . 27

3.4 .3 Sensor/Message Display . . . . . . . . . . . . . . . . . . . 28

3.4 .4 Data Manager . . . . . . . . . . . . . . . . . . . . . . . . . . . . . . . . . 29

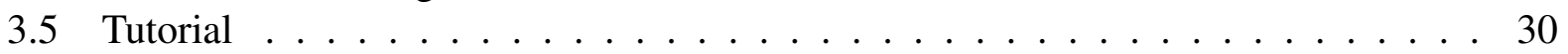


3.6 Chapter Summary . . . . . . . . . . . . . . . . . . . . . . . . 30

Chapter 4 Human Subject Experiment . . . . . . . . . . . . . . . . . . . . . . 31

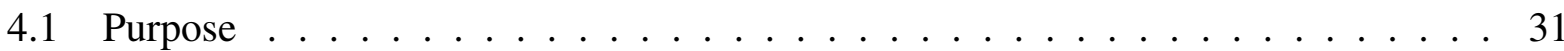

4.2 Experimental Apparatus . . . . . . . . . . . . . . . . . . 32

4.3 Experimental Approach . . . . . . . . . . . . . . . . . . 32

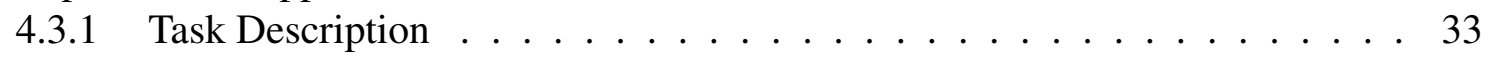

4.3.2 Multitasking and Heads-up Awareness . . . . . . . . . . . . . . . . . . 34

4.3.3 World Difficulty Classification . . . . . . . . . . . . . . . . 35

4.3.4 Situation Awareness Measurement . . . . . . . . . . . . . 35

4.4 Pilot Study . . . . . . . . . . . . . . . . . . . . . . . . . . 37

4.5 Experimental Design . . . . . . . . . . . . . . . . . . . . . . . . . . . . . . . . . . . .

4.5 .1 Factors and Levels . . . . . . . . . . . . . . . . . . . . . . . . . . . . . . . . . . . . . . . . . . . . .

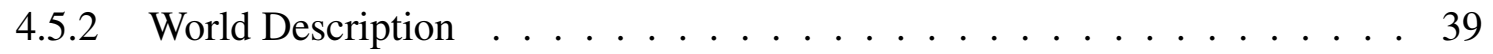

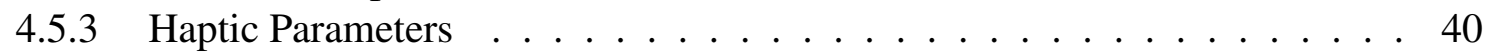

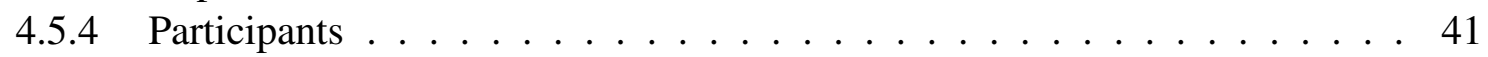

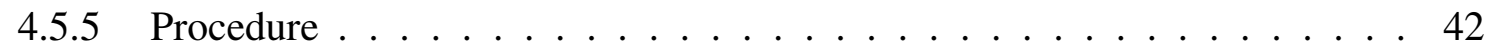

4.6 Measures . . . . . . . . . . . . . . . . . . . . . 42

4.7 Data Generator . . . . . . . . . . . . . . . . . . . . . . . . . . . . . . . . . . . . .

4.8 Data Viewer . . . . . . . . . . . . . . . . . . . . . 45

4.9 Chapter Summary . . . . . . . . . . . . . . . . . . . . . . 46

Chapter 5 Results and Discussion . . . . . . . . . . . . . . . . . . . 47

5.1 Primary Measures . . . . . . . . . . . . . . . . . . . . . . . . 48

5.1 .1 Percent Hotspots Found . . . . . . . . . . . . . . . . . . . 48

5.1 .2 Total Command Score . . . . . . . . . . . . . . . . 50

5.1 .3 Total SA Score . . . . . . . . . . . . . . . . 51

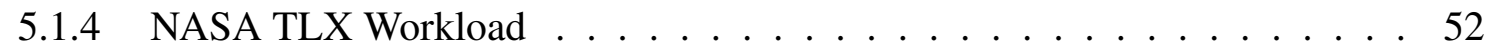

5.2 Secondary Measures . . . . . . . . . . . . . . . . . . 53

5.2 .1 Shape Time Percentage . . . . . . . . . . . . . . . . 53

5.2 .2 Percent Hotspots Found by Shape . . . . . . . . . . . . . . . . . 54

5.2 .3 Shape Hotspots Found Rate . . . . . . . . . . . . . . . . 56

5.2 .4 Spacing Time Percentage . . . . . . . . . . . . . . . . 57

5.2 .5 Spacing Hotspots Found Rate . . . . . . . . . . . . . . . . . 58

5.2 .6 Hotspots Found Rate . . . . . . . . . . . . . . . . . . . 59

5.2 .7 Average Search Time . . . . . . . . . . . . . . . . . 60

5.2 .8 Average Manipulation Time . . . . . . . . . . . . . . . . . . . 61

5.2.9 Average Team Command Response Time . . . . . . . . . . . . . . . . . 62

5.2.10 Average SA Response Time . . . . . . . . . . . . . . . . . . 63

5.2 .11 SA Accuracy Score . . . . . . . . . . . . . . . . . . . 64

5.3 Results Summary . . . . . . . . . . . . . . . . . . . . . . . . . . . . . . . . . 65

5.4 Chapter Summary . . . . . . . . . . . . . . . . . . . 66

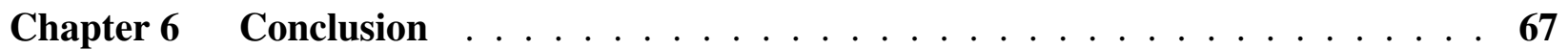

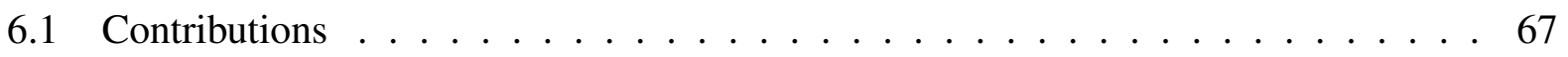


6.1.1 Shape-based Robot Team Interaction . . . . . . . . . . . . . . . . . . 67

6.1 .2 Novel Haptic Feedback Mapped to Team Modes . . . . . . . . . . . . . . . 68

6.1 .3 Experimental Simulation Test Bed . . . . . . . . . . . . . . 69

6.2 Experimental Results . . . . . . . . . . . . . . . . . . . . . 69

6.3 Future Work . . . . . . . . . . . . . . . . 70

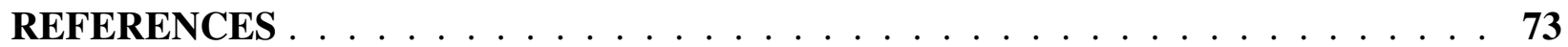




\section{LIST OF TABLES}

4.1 Haptic feedback parameters, consisting of stiffness and damping coefficients. . . . 41

5.1 Effect $p$ values for all measures. Significant effects are in bold. . . . . . . . . . . . 47

5.2 Significant differences for percent hotspots found . . . . . . . . . . . . . . 48

5.3 Significant differences for total SA score. . . . . . . . . . . . . . 51

5.4 Significant differences for NASA TLX workload. . . . . . . . . . . . . . . . 52

5.5 Significant differences for shape time percentage. . . . . . . . . . . . 53

5.6 Significant differences for percent hotspots found by shape. . . . . . . . . . . . 54

5.7 Significant differences for shape hotspots found rate. . . . . . . . . . . . . . . . . . . . . . . 56

5.8 Significant differences for hotspots found rate . . . . . . . . . . . . . . 59

5.9 Significant differences for search time. . . . . . . . . . . . . . . . . 60

5.10 Significant differences for manipulation time. . . . . . . . . . . . . . . . 61

5.11 Significant differences for SA accuracy score . . . . . . . . . . . . . . . 64 


\section{LIST OF FIGURES}

2.1 Travel and surround mode visualization . . . . . . . . . . . . . . . . 9

2.2 Visualization of ground force feedback, $\mathbf{f}_{g} \ldots \ldots \ldots \ldots$

2.3 Creating a surrounding shape via force node network $\ldots \ldots \ldots 13$

2.4 Interaction between haptic cursor and force node network. . . . . . . . . . . . 15

2.5 Views of team shape, before and after shape manipulation. . . . . . . . . . . 16

2.6 Views of robots within the team shape, before and after spacing manipulation. . . . 17

2.7 Views of robot team, before and after a relative travel command. . . . . . . . . . 20

2.8 Travel force node network using convex hull of robot positions. . . . . . . . . . . 21

2.9 Side-view of interaction with convex hull surface during travel mode. . . . . . . . 21

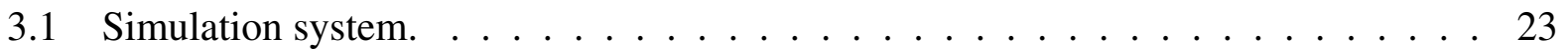

3.2 Simulation system overview. . . . . . . . . . . . . . . . . . . . . . . . 24

3.3 Novint Falcon. . . . . . . . . . . . . . . . . . . . . . . . . . 25

3.4 Team display, showing environment, robot team, and haptic cursor. . . . . . . . . . 27

3.5 Found and expired hotspots visualization. . . . . . . . . . . . . . . . . 28

3.6 Sensor/Message display, showing team sensor readings. . . . . . . . . . . . . . . 29

3.7 RoTHSim Tutorial screenshots. . . . . . . . . . . . . . . . . . . . . . . . 30

4.1 Dual-monitor, multimodal workstation. . . . . . . . . . . . . . . . . 32

4.2 Easy and hard world views. . . . . . . . . . . . . . . . . . . 40

4.3 Search and manipulation time visualization. . . . . . . . . . . . . 43

4.4 Data Generator screenshot. . . . . . . . . . . . . . . . . . . . . . . . 45

4.5 Data Viewer screenshot. . . . . . . . . . . . . . . . . . . . . . 46

5.1 Boxplots (a) and mean/standard deviation plots (b) for percent hotspots found . . . 49

5.2 Boxplots (a) and mean/standard deviation plots (b) for total command score . . . 50

5.3 Boxplots (a) and mean/standard deviation plots (b) for total SA score . . . . . . . . 51

5.4 Boxplots (a) and mean/standard deviation plots (b) for NASA TLX workload . . . 53

5.5 Boxplots (a) and mean/standard deviation plots (b) for shape time percentage . . . 54

5.6 Boxplots (a) and mean/standard deviation plots (b) for percent hotspots found by shape . . . . . . . . . . . . . . . . . . 55

5.7 Boxplots (a) and mean/standard deviation plots (b) for shape hotspots found rate . . 57

5.8 Boxplots (a) and mean/standard deviation plots (b) for spacing time percentage . . 57

5.9 Boxplots (a) and mean/standard deviation plots (b) for spacing hotspots found rate . 58

5.10 Boxplots (a) and mean/standard deviation plots (b) for hotspots found rate . . . . 59

5.11 Boxplots (a) and mean/standard deviation plots (b) for search time $\ldots \ldots \ldots$. . . . 60

5.12 Boxplots (a) and mean/standard deviation plots (b) for manipulation time . . . . 61

5.13 Boxplots (a) and mean/standard deviation plots (b) for team command response time 62

5.14 Boxplots (a) and mean/standard deviation plots (b) for SA response time . . . . 63

5.15 Boxplots (a) and mean/standard deviation plots (b) for SA accuracy score . . . . . 64 


\section{NOMENCLATURE}

$b_{s} \quad$ Damping coefficient for spacing manipulation

$\mathbf{C O M}_{n} \quad$ Nodal network center of mass location

$d \quad$ Euclidean distance from a robot sensor to a hotspot

$d_{h} \quad$ Hotspot group difficulty value

$\delta_{\text {spacing }} \quad$ Distance between neighboring force nodes

$\mathbf{e}_{c} \quad$ Convex hull penetration vector

$\mathbf{e}_{i} \quad$ Penetration vector for the $i$-th contacted force node

$\mathbf{e}_{g} \quad$ Ground surface penetration vector

$k_{c} \quad$ Convex hull stiffness coefficient

$k_{g} \quad$ Virtual ground stiffness coefficient

$k_{n} \quad$ Force node stiffness coefficient

$k_{s} \quad$ Stiffness coefficient for spacing manipulation

$\mathbf{f}_{c} \quad$ Feedback force due to interaction with the virtual convex hull surface

$\mathbf{f}_{e} \quad$ External force applied to a force node

$\mathbf{f}_{g} \quad$ Feedback force due to virtual ground surface

$\mathbf{f}_{n} \quad$ Feedback force due to interaction with force nodes

$\mathbf{f}_{r} \quad$ Feedback force provided relative location between the haptic cursor and the team in travel mode

$\mathbf{f}_{s} \quad$ Feedback force due to spacing manipulation

$\mathbf{F}_{s h} \quad$ Total feedback force during shape manipulation

$\mathbf{F}_{r} \quad$ Total feedback force due to relative travel force during travel mode

$\mathbf{F}_{s} \quad$ Total feedback force during spacing manipulation

$\mathbf{F}_{t} \quad$ Total feedback force during travel mode, including relative travel and shape exploration forces

$H \quad$ Number of hotspots in a group used to compute group difficulty

$L_{i} \quad$ Equilibrium spacing distance between the $i$-th set of consecutive robots in a surrounding formation

$M \quad$ Total number of robots in a surrounding formation

$n \quad$ Number of robot nodes away from the one closest to the haptic cursor during spacing manipulation

$r \quad$ Distance of haptic cursor from $\mathbf{C O M}_{n}$ in spacing manipulation

$r_{\max } \quad$ The maximum control distance away from $\mathbf{C O M}$ during spacing manipulation

$r_{\text {min }} \quad$ The minimum control distance away from $\mathbf{C O M}_{n}$ during spacing manipulation

$r_{n} \quad$ Force node radius

$\rho_{c} \quad$ Equilibrium distance factor for the closest robot in a surrounding formation

$\rho_{i} \quad$ Equilibrium distance factor for the $i$-th robot in a surrounding formation

$\rho_{\max } \quad$ Maximum equilibrium distance factor used to determine $\rho_{i}$ values

$\mathbf{p}_{\text {cursor }}$ Position of the haptic cursor in virtual world coordinates

$\mathbf{p}_{r} \quad$ Response location chosen by the operator

$p_{d} \quad$ Hotspot group active duration factor used to compute group difficulty

$p_{f} \quad$ False positive rate for hotspot detection

$p_{h} \quad$ Hotspot group size factor used to compute group difficulty

$p_{\max } \quad$ Maximum true positive rate for hotspot detection 
$p_{\min } \quad$ Minimum true positive rate for hotspot detection

$p_{t} \quad$ True positive rate for hotspot detection

$P_{n} \quad$ Total nodal network perimeter

$s_{r t} \quad$ Response time score computed from response time

$\sigma \quad$ Standard deviation of the Gaussian curve used in defining true positive rate

$t_{\max } \quad$ Maximum allowable response time used in computing response time score

$t_{r} \quad$ Operator response time to incoming messages or commands

$\tau_{\max } \quad$ Maximum active duration value used to compute group difficulty

$\tau_{\min } \quad$ Minimum active duration value used to compute group difficulty

$v_{\max } \quad$ Maximum allowable deviation value used to compute response accuracy

$v_{c} \quad$ The correct response value used to compute response accuracy

$\mathbf{v}_{\text {cursor }} \quad$ Velocity of the haptic cursor in virtual world coordinates

$v_{r} \quad$ Operator response value used to compute response accuracy 


\section{CHAPTER 1. INTRODUCTION}

There are many current and future scenarios in which a human must manage a team of air, ground, and humanoid robots. Some of these scenarios include wilderness search-and-rescue [1], rescue operations in buildings damaged by fire or earthquake $[2,3]$, searching of a building by law enforcement agencies [4], pollution monitoring and clean-up [5], and military patrol and cordon operations in an urban environment [6]. In each scenario, the robot team serves as an extension of the operator's ability to gather information in complex and often dangerous environments. Enabling the human operator to manage the robot team in an intuitive, effective, and time-efficient manner is therefore critical to the success of operations involving robot teams.

The state of the art in controlling autonomous robots (in use by current military, law enforcement, and search-and-rescue agencies) is for a single robot to be controlled and monitored by one or many human operators (see, for example, [7]). This interaction model is clearly not ideal if the objective of employing autonomous robots is to augment the capabilities of humans and maximize the information-gathering capabilities of the team $[10,11]$. A preferable interaction model is for a single human operator to control multiple autonomous robots $[8,9]$. The effectiveness of such an approach is limited by the operator's ability to command the actions of multiple agents and receive information about the state of the robot team, while accomplishing a primary task, whether it be search-and-rescue, surveillance, etc.

When the robot team possesses appropriate autonomy for the given scenario, the problem becomes one of "team management" rather than "robot control," enabling the operator to focus on task objectives and interpretation of gathered data, rather than on the robots. If control of the robot team could be almost second-nature then a more "heads-up" approach to operating the team would be possible. The phrase "heads-up" is defined in this thesis as demonstrating a sufficient level of competency in a single task to focus attention elsewhere on other important or urgent tasks. An operator who is "heads-down" tends to focus solely on a single task and may experience 
difficulty in responding quickly or accurately to additional tasks that arise. In a human-robot interaction scenario, this could occur when an operator is so focused on a graphical user interface that awareness of surroundings or response to incoming commands is degraded. While considering a single-operator-multiple-robot interaction, enabling the operator to achieve heads-up control is challenging.

In general, when multiple tasks demand attention from the same sensory channel, interference can degrade performance or possibly result in task overload [12]. The operator's ability to multi-task could be improved with the use of a multimodal interface, using both visual and haptic feedback. Haptic feedback has been shown to reduce collisions when piloting individual robotic vehicles $[13,14]$, and provide a sense of team-level properties when teleoperating multiple robots [15-17]. There are, however, possible drawbacks to using haptic feedback, as it has been shown to increase operator workload in some studies [18, 19].

The objective of this research is to investigate the effects of haptic feedback on heads-up control, situation awareness and workload while managing a small team of robots in reconnaissance and surveillance tasks. Human-subject experiments are conducted to verify the effectiveness of this approach, by analyzing changes in workload, situation awareness, and other measures.

\subsection{Background/Related Work}

This section will discuss, in greater detail, the related research in the areas of swarm robotics, haptic feedback, situation awareness and workload.

\subsubsection{Swarm Robotics}

As previously mentioned, before a human or multiple humans can be expected to manage multiple robots at once, a firm understanding of team control needs to be developed. Therefore, it is important that models used to govern robot team behavior are ones that operators can intuitively understand. There are many examples in nature of swarms of ants, fish or bees that work toward a common goal by creating distinct formations [20]. For this reason, models of robot teams are often focused on bio-inspired, collective behaviors such as swarming, flocking, foraging or colonybuilding [21]. It is not expected that operators have a deep understanding of swarm formations that 
occur in nature to be successful in human-swarm interaction. Bio-inspired swarm models simply provide insight into how a team of robots can be controlled in a distributed way. Most models are also designed with an aim for simplicity of individual behavior and local communication between robots [22]. A simple model helps the operator recognize the team intent and thereby interacting easier with the team.

The main contributions of this work are not focused on the development of novel robot team behavior, but on the development of haptic-based user interaction, designed to interface with an existing model of robot team behavior. The model of choice for this thesis has been developed by Kris Alder, which will be discussed in Section 2.1 [23].

\subsubsection{Haptic Feedback}

Haptic feedback has been used in the teleoperation of individual UAVs to help avoid obstacles in indoor environments [13, 18, 24]. In 2014, Philbrick et al. [14, 25] presented two novel force feedback algorithms which provided correction forces that opposed the direction of nearby obstacles. One algorithm used time to impact as a proportional model for the magnitude of the force, and the other used a dynamic virtual spring model to connect artificial springs to the walls of an indoor environment, thereby stabilizing the device end effector based on changes in position and velocity from a home position. These algorithms were tested experimentally along with two other novel audio feedback algorithms in a multimodal user interface. Results showed a decrease in number of collisions and collision time with haptic feedback enabled. The audio feedback was neither helpful nor harmful to operator performance, although many participants in the study noted that with practice, it could become useful.

Haptic feedback algorithms specific to swarm behavior have also been developed and tested. Nunnally et al. [15] presented a method of navigating a decentralized swarm of ground robots using the displacement of a haptic device as input. The haptic feedback received by the

operator was a function of that displacement and each individual robot was acted upon using that force as a component to robot control. Other components of the robot control algorithm modeled autonomous behavior through attractive or repulsive forces, which acted to maintain cohesiveness in the swarm. A human subject experiment was conducted that tested an operator's ability to find hidden targets as quickly as possible while maintaining swarm connectivity. In one environ- 
ment (Math), to test the effects of a brief loss in visual perception of the team, simple algorithmic problems were presented on screen, blocking the view of the swarm until the correct answer was entered. This condition was presented to the operator to simulate a situation in which navigation became a secondary task, and a primary task needed full visual of the display, as is the case if video-surveillance or monitoring an electronic feed was occasionally required. Results showed that haptic feedback was marginally significant in improving target efficiency (targets/time) in the Math environment and statistically significant in increasing swarm ending connectivity.

Researchers at the Army Research Laboratory (ARL) have developed multimodal interfaces (with visual, audio and tactile feedback) to test a soldier's ability to identify points of interest and respond quickly to incoming commands, while monitoring and controlling a swarm of UAVs that follow a convoy [26]. The swarm behavior is modeled to maintain a perimeter or cordon around the convoy as it travels through waypoints and perform reconnaissance of the points of interest chosen by the operator. In experimental studies, measures of response time to incoming commands were found to decrease with the presence of tactile or audio feedback compared to visual feedback alone. One of their points of future work was to incorporate methods to allow the soldiers to adjust the behavior of the swarm.

Other studies have shown that small teams of robots have benefited from using haptic feedback in methods of controlling a group of UAVs in a cluttered environment [27], and conveying a sense of team-level properties such as manipulability [17]. Overall, the potential for using haptic feedback to control a robot team, maintain awareness of primary tasks and respond to secondary tasks, demands further research.

\subsubsection{Situation Awareness}

An extensive review on humans' involvement in multirobot control has shown that Situation Awareness (SA) is an essential measure when investigating an operator's ability to supervise and manage a robot team [28]. The Endsley definition of SA is "the perception of the elements in the environment within a volume of time and space, the comprehension of their meaning, and the projection of their status in the near future" [29]. Although Endsley's methodology has been questioned over the years [30,31], her definition remains appropriate and valuable to many research areas in which it is used [32]. 
There have been several techniques developed with the intent of effectively measuring SA. One such method is Endsley's Situation Awareness Global Assessment Technique (SAGAT) [29], which was originally designed to measure SA of a pilot in a cockpit simulation. This method provides a means of objective measurement, but requires extensive training and halts the simulation to probe an operator with specific questions. Another method is the Situational Awareness Rating Technique (SART), which is a post trial subjective rating technique [33]. Both SAGAT and SART have been validated in their own right, however, research has shown little correlation between the two methods [34]. This raises the question to whether they are measuring SA, an operator's perception of SA, or something else entirely.

Other more recent measurement techniques have also been developed, like the Situation awareness-based Agent Transparency model (SAT), which uses the Endsley definition of SA as a model to measure interface transparency [35]. Although SAT does not aim to directly measure operator SA, it does show how the Endsley model has been used in current research. In addition to these methods, several main categories of SA metrics have been proposed, which include measures of effectiveness, performance, memory recall, reaction strength and subjective ratings [36]. A few of these metrics will be used in considering a method to evaluate an operator's SA, explained in Section 4.3.4.

\subsubsection{Workload}

In experiments involving haptic feedback and teleoperation, the tasks themselves or the type of robotic controller used may cause the operator to experience a noticeable level of workload. Workload can be defined as the task demands (physical, mental and emotional) on human operators while interacting with robots or machines [37]. In general, one can assume to see a noticeable decrease in performance as operator workload increases. One of the purposes of this research is to determine how haptic feedback affects workload. Therefore, it is important to have a wellestablished technique for measuring workload to properly investigate this claim. Some of the most well-known methods that support quantitative measurement of workload are the dual task method, physiological signals and subjective evaluation [38].

The dual task method requires that two tasks be performed simultaneously, one being the primary task and the other an easier secondary task. The operator's performance in the secondary 
task is used as a measure of workload [39]. This research involves using a secondary task to measure SA (See Section 4.3.4), and the task is not considered easy. For this reason, the dual task method seems ill-suited for this research. Physiological signals, such as pulse rate, eye movement, skin temperature, etc., could be used to indicate high levels of workload. Several of these measures have led to significant differences in cognitive workload in haptic human-robot interactions [38]. Although potentially useful for obtaining an objective measure for workload, this method requires special equipment that is either expensive or not readily available. Other methods have been developed to objectively measure workload in real-time using behavioral entropy [25]. This method is unobtrusive and calculates deviations from an operator's predicted device movement to calculate a workload rating.

Due to there being more focus on new measurement methods of SA in this research, implementing behavioral entropy was not first priority. Thus, subjective measurement of workload was a preferable choice. A common subjective evaluation method is the NASA Task Load Index (NASA TLX). After completion of a task, the operator performs a self-rating using a multi-point scale. A workload score is generated from a weighted-average of six sub scales [40]. Although subjective evaluations are often scrutinized, the NASA TLX is widely accepted in the field of human-robot interaction and will be used in this research.

\subsection{Contributions}

This thesis adds to work previously done with the following contributions:

- Shape-based robot team interaction. A human operator interacts with an autonomous team of robots in cordon and patrol by treating the team as a deformable shape that can be manipulated to distribute the team as desired. This approach is designed for intuitive and heads-up control of the entire team instead of individual robots in the team.

- Novel haptic feedback mapped to team modes. Several haptic feedback algorithms were developed to provide the operator with team formation and distribution information in a variety of team control modes. Effects on operator situation awareness and workload are compared with and without feedback. 
- Experimental simulation test bed. A robust experimental simulator was developed to accurately measure an operator's ability to maintain heads-up awareness and control of a small team of robots.

\subsection{Thesis Overview}

The remainder of this thesis will be organized as follows:

- Chapter 2, Team Interaction and Haptic Feedback Development, describes the development and implementation of team control modes and novel feedback algorithms.

- Chapter 3, Simulation System and Interface, explains the features and development of the simulation system.

- Chapter 4, Human Subject Experiment, presents the design of a user study.

- Chapter 5, Results and Discussion, reviews the results of the human subject experiment.

- Chapter 6, Conclusion, gives final remarks and future work. 


\section{CHAPTER 2. TEAM INTERACTION AND HAPTIC FEEDBACK DEVELOPMENT}

The challenge for a human operator is to manage the team of autonomous robots effectively, without being overburdened or losing SA through "heads-down" attention focused on a graphical user interface. Without the haptic channel, an operator could only receive feedback from the system via the visual or audio channels. Past research has shown that audio feedback methods may be difficult to interpret and may only improve the operator's performance after extensive training [14]. Although audio feedback has potential, this work specifically focuses on the effects of haptic feedback. Our approach involves augmenting visual stimuli with haptic feedback, with the intent of helping the operator divide the task load between visual and haptic channels. This chapter presents the robot behaviors model and the kinesthetic haptic feedback which has been developed to support our approach.

\subsection{Robot Behaviors Model}

The robot behaviors that govern the robot team's movement were developed by Kris Alder [23]. The model assumes a military patrol and cordon scenario, although the interaction approach will be generalizable to other scenarios that require a human operator to control the movement and distribution of autonomous robots. In this scenario, the operator uses the team to search around buildings of interest in an urban environment. Only the exterior regions of the building are considered while searching. The robots themselves are modeled as autonomous, omni-directional agents, and for simplicity, their movement is planar in $x$ and $y$. The following sections will describe the different team modes associated with the patrol and cordon scenario, what information is provided to the user interface to virtually render the team, and what commands are sent to the model to manipulate the team. 


\subsubsection{Team Modes}

There are two modes available to the team, travel and surround. Graph theory is used to describe the relationships between robots, with each robot corresponding to a node in the graph. An example formation for each mode is shown in Figure 2.1.

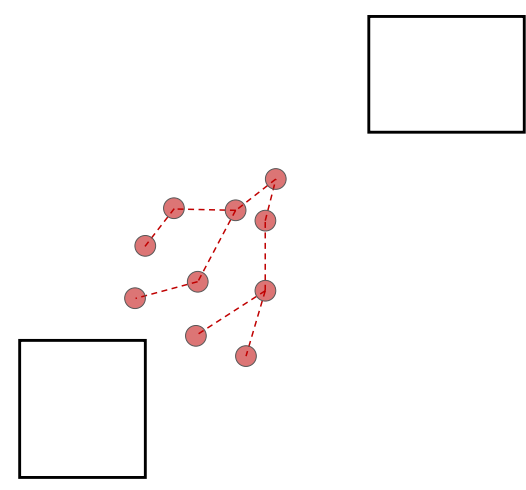

(a) Travel mode

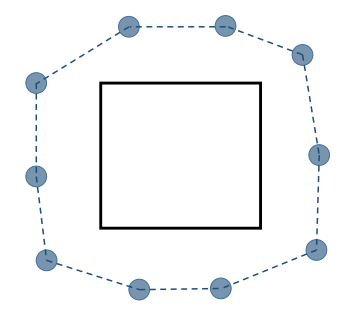

(b) Surround mode

Figure 2.1: In each image, individual circles are graph nodes (representative of robots), and dotted lines are graph connections. In (a), the travel mode is represented by a tree formation as the team travels between two buildings. In (b), the surround mode is represented as a spanning ring formation that surrounds one building.

A travel is used when the team is traveling between buildings, and represents the patrol action. During travel mode, the team forms a spanning forest, governed by the graph connections, where each robot follows another lead robot. This allows the robots to obey forces that repel them from the current building and travel to a new one. While this happens, the team attempts to stay close together, until a nearby building is identified and they switch to a surround mode. In some circumstances, some robots will get stuck around corners while the rest of the team travels to the new location. Upon arrival at a building of choice, trailing robots may unintentionally become attracted to another building, due to their distance away from the rest of the team. These are limitations of the current robot behavior, but they can be easily remedied by applying several travel commands instead of just one (See Section 2.6).

A surround is used when the team is surrounding a building, and represents the cordon action. During surround mode, the team forms a spanning ring around a building, allowing the model to robustly determine a surrounding shape, regardless of a convex or concave shape of the 
building. The transitions between these two modes are handled by the robot behaviors model. In either mode, the individual robots are attracted to nearby buildings, but repelled by the boundaries they form to prevent the team from passing through buildings.

\subsubsection{Team Information and Control}

The state of each robot and the team as a whole are generated by the robot behaviors model. Access to this information is provided to allow the development of a user interface. There are also several methods of interaction, to manipulate the team and update robot state information based on user input. These methods are listed as follows:

- Travel command This tells the model that a new travel location is present, which prompts the team to attempt to travel to that location.

- Inter-robot Radius Each robot has a minimum radius that it uses to search for nearby robots and form connections between them. In travel mode, this value is decreased to allow closer connections while traveling. In cordon mode, it is increased to spread our further and more quickly surround a building.

- Force command External forces can be set on each individual robot. When desired positions are calculated, a proportional controller uses this force command several times to guide each robot to a desired position.

\subsection{Modeling Clay: A Haptic Metaphor}

Based on the results of a brainstorming breakout session at the 2012 AAAI Fall Symposium on Human Control of Biological Swarms, Diana et al. proposed the idea of using a deformable medium, such as modeling clay, as a "joystick" to command the distribution of large-scale swarmlike teams of homogeneous vehicles [41]. They demonstrated a molding scheme in which an operator formed modeling clay into various shapes in the view of an overhead camera and a team of micro robots replicated the formation commanded by the shaped clay. We modify the modeling clay metaphor so that a human can shape the distribution of robot teams by manipulating a virtual deformable volume through stretching, pulling and other operations. The modeling clay metaphor 
forms the basis for the haptic sensations that the user feels while distributing the robot team. Note that, unlike the work in [41], physical modeling clay is not used in our method; the concept and physics of modeling clay are used to generate the visual and haptic representation of the robot team.

\subsubsection{Modes of Interaction}

Similar to how movement of a physical hand or finger changes the shape of physical modeling clay, a virtual haptic cursor may be used to change the shape of virtual modeling clay. This direct analogy is used to map the position of a desktop haptic device end effector to a virtual cursor position shown on a graphical user interface. The operator uses the haptic cursor to experience each of the modes of interaction, where each mode has its own type of haptic feedback. Choosing each mode of interaction is done by engaging specific buttons on the haptic device, namely SHAPE, SPACING and TRAVEL buttons. A more in-depth description of these buttons will be covered in Section 3.2.

During surround mode there are three ways in which the operator can position the haptic cursor to interact with the team. The methods are listed below and described in greater detail in Section 2.5.

- Shape exploration Movement of the haptic cursor near the team results in feedback designed to inform the operator of team shape.

- Shape manipulation (SHAPE) The team shape is manipulated through stretch, push or pull operations of the haptic cursor, thereby changing the positions of particular robots in the team.

- Spacing manipulation (SPACING) The spacing between robots within the team shape changes based on the location of the haptic cursor. The operator may gesture toward a portion of the shape and the robots will attempt to form more densely near that location.

Although not a mode of interaction that involves haptic feedback, the operator may also double-click the SHAPE button to reset the team shape to the surrounding formation that the team originally made. 
While in travel mode, only the autonomous behavior of the team is considered for team movement, so the operator simply needs to specify the travel location. The travel location is chosen by pressing and holding the TRAVEL button, moving the haptic cursor to a location in the environment and then releasing the button. Two types of force feedback during travel mode are explained in Section 2.6.

\subsection{Ground Force Feedback}

Because the haptic interface is capable of 3D motion but the robot team only travels within a plane, a virtual ground surface is present to help identify the plane in which the team movement occurs. There is a visual change in cursor color when in contact with the ground surface but the presence of force feedback is also an indicator of ground contact. The ground force feedback is modeled as a virtual spring and can be expressed as

$$
\mathbf{f}_{g}=-k_{g} \mathbf{e}_{g}
$$

where $k_{g}$ is the virtual ground spring constant and $\mathbf{e}_{g}$ is the penetration vector of the haptic cursor with the ground surface. In essence, a force is exerted on the user's hand to prevent the haptic cursor from descending below the ground plane. A visualization of this equation is shown in Figure 2.2.

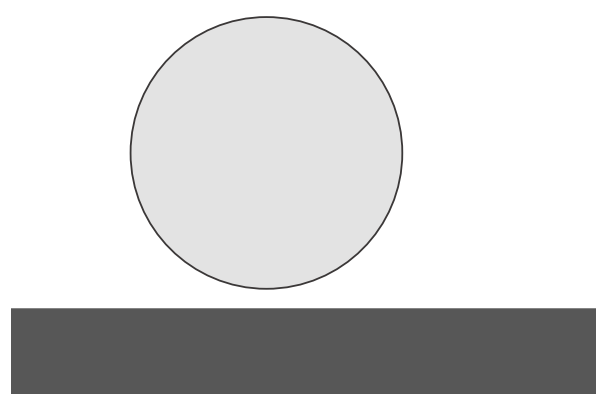

(a) No contact with ground surface

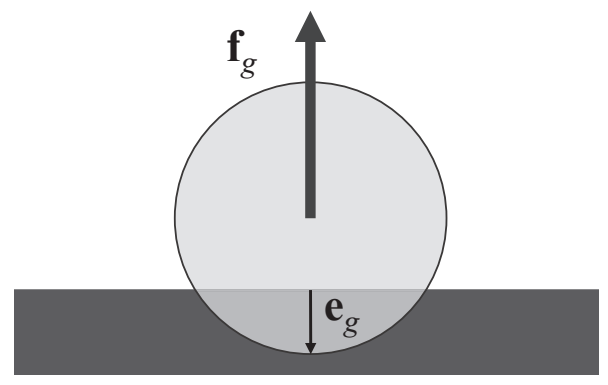

(b) Ground force feedback produced

Figure 2.2: Visualization of ground force feedback, $\mathbf{f}_{g}$ 


\subsection{Force Node Network}

A discrete approximation of the continuous clay metaphor is created by placing potential force field spheres at the location of each of the robots in the spanning ring and at multiple points between robots. In essence, these potential spheres form the nodes of a "force graph" on which the haptic interaction forces and graphical representation of the deformable volume ("virtual modeling clay") are based. An example of how the force nodes span between robots is shown in Figure 2.3. Notice that some force nodes are specifically assigned to a robot location.

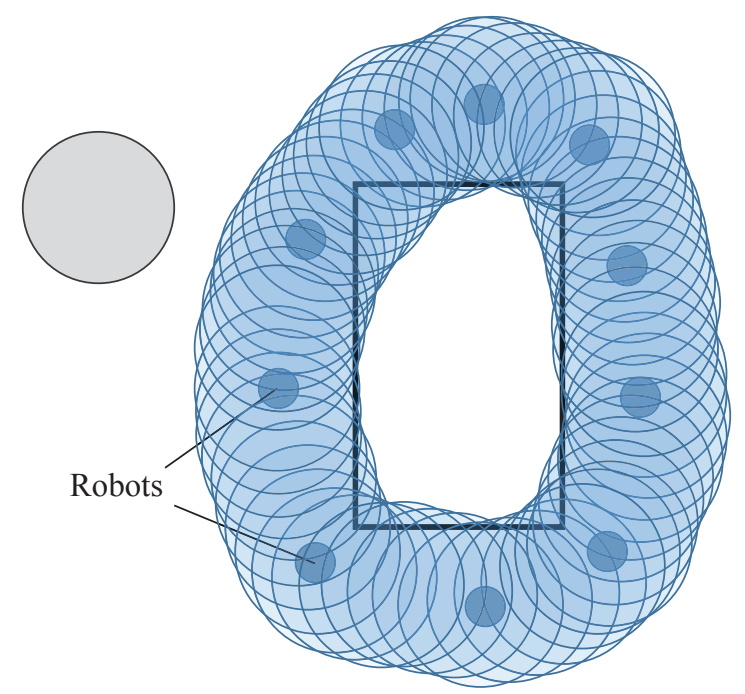

Figure 2.3: This image shows how force nodes (light blue) create the spanning ring shape after the team (dark blue) has surrounded a building.

To develop a dynamic system that the operator can manipulate, the potential spheres also form the mass nodes of a virtual mass-spring-damper network. Frictional forces are also used to model plastic deformation of the volume. Each force node in the network is positioned a distance

$$
\delta_{\text {spacing }}=r_{n} / 4
$$

from neighboring nodes, where $r_{n}$ is the node radius. In other words, nodes are placed close enough together to cause eight consecutive nodes to overlap. Parameter values for mass, spring, and damping constants were subjectively chosen and allow each node to maintain sufficient distance relative to neighboring nodes, stabilize the network and create a distinguishable volume with which to in- 
teract. Similar to the team behavior model, nodes are placed along the two-dimensional ground surface, as to replicate the effect of deformable clay being placed on a flat surface. Each node has a $z$-position of zero, such that one hemisphere is above the surface, and the other hemisphere is below the surface. The robot locations that define the deformable ring shape are determined by the current mode, and will be discussed in sections 2.5 and 2.6. The deformable ring forms the basis for computing the haptic feedback force felt by the operator.

\subsection{Surround Interaction}

In surround mode, there are three types of interaction modes with corresponding force feedback algorithms, namely, shape exploration, shape manipulation and spacing manipulation. Each mode is designed to prevent the operator from needing to micromanage each robot, but instead focus on collective, team-level control. A description of each mode is presented in this section.

\subsubsection{Shape Exploration}

When the haptic cursor comes into contact with the boundary of force nodes during shape exploration, there is no manipulation of the shape. The force nodes are fixed and the operator cannot cause them to move during contact, but force feedback is still provided. The force feedback is computed as a function of penetration distance on each of the nodes with which there is contact. Thus, the nodal force feedback during this mode is given by

$$
\mathbf{f}_{n}=-\sum_{n=1}^{N} k_{n} \mathbf{e}_{n}
$$

where $k_{n}$ is the node stiffness coefficient and $\mathbf{e}_{n}$ is the penetration vector for the $n$-th contacted node. Accounting for ground feedback as well, the total haptic feedback force in shape exploration is

$$
\mathbf{F}_{s h}=\mathbf{f}_{g}+\mathbf{f}_{n}
$$

When an operator uses the haptic cursor to explore along the boundary of the nodal network, it may be preferred to contact the ground surface as well. This allows for the cursor to remain in 
the same plane in which the team shape exists and provide a stable position for the haptic cursor as it rests against both the ground and nodal network surfaces. A visualization of the feedback produced by coming in contact with a set of fixed force nodes is shown in Figure 2.4.

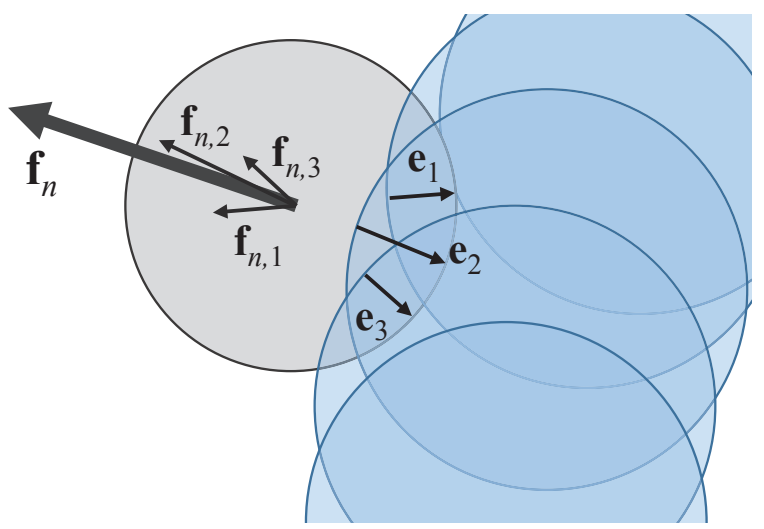

Figure 2.4: A haptic feedback force, $\mathbf{f}_{n}$, is produced due to interaction between the haptic cursor and several force nodes.

\subsubsection{Shape Manipulation}

During shape manipulation, portions of the nodal network move away from the haptic cursor when contacted, as shown in Figure 2.5. The robot positions are then updated from the manipulated robot node positions, which are governed by the dynamics of the entire network. This method allows the operator to quickly move multiple robots in the team without needing to interact with each robot individually.

The force feedback is governed by the same equations presented in shape exploration, except external forces are applied to the nodal network. These external forces are of equal magnitude and opposite direction of the nodal force feedback, thus $\mathbf{f}_{e, i}=-\mathbf{f}_{n, i}$. The force feedback is designed to provide inertial forces similar to what one would feel while plastically deforming physical modeling clay. To simulate this, the operator must use the haptic cursor to interact with the shape and produce external forces that exceed the frictional forces included in the nodal network dynamic model. If penetration of the haptic cursor into the contacted force nodes is too small, no or little movement of the shape will result. The frictional forces are not strong enough to cause difficulty in making adjustments, so the operator may manipulate the shape just as well with force feedback 


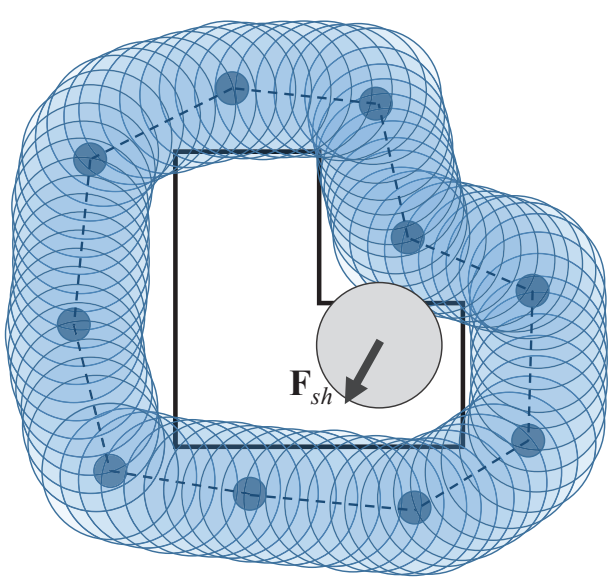

(a) Initial team shape

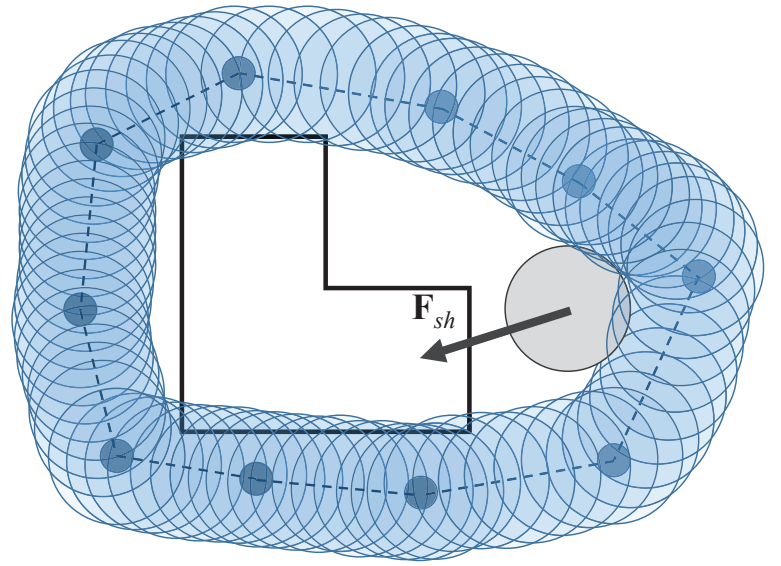

(b) Team shape after manipulation

Figure 2.5: This figure shows views of the team shape before (a) and after (b) shape manipulation. The operator experiences a feedback force, $\mathbf{F}_{s h}$, in each case.

as without it. The frictional forces are strong enough to allow for plastic deformation, thereby saving the previous shape manipulation performed by the operator. Therefore, the operator may manipulate the shape multiple times or in various parts and these adjustments will be recognized by the dynamic nodal network model.

\subsubsection{Spacing Manipulation}

The autonomous behavior of the team creates an initially uniform distribution, meaning that each robot maintains an equal distance from neighboring robots throughout the spanning ring shape. This method of interaction allows the operator to move robots closer together in some portions of the shape and farther away from each other in others. The proposed method allows the operator to simply gesture toward a portion of the team with the haptic cursor and the robots will concentrate more toward the gesture direction. An example of spacing manipulation is shown in Figure 2.6 and the meaning of several variables on the figure will be explained in the following paragraphs.

Since each robot is assigned a node in the network, neighboring nodes act as potentially new locations for each robot. Given a team of $M$ robots, each robot node is labeled from 1 to $M$ in a counterclockwise direction around the surrounding shape. The robot spacing within the shape is modeled as a set of equilibrium distances, $L_{i}$, that act in the discrete space of the nodal network. 


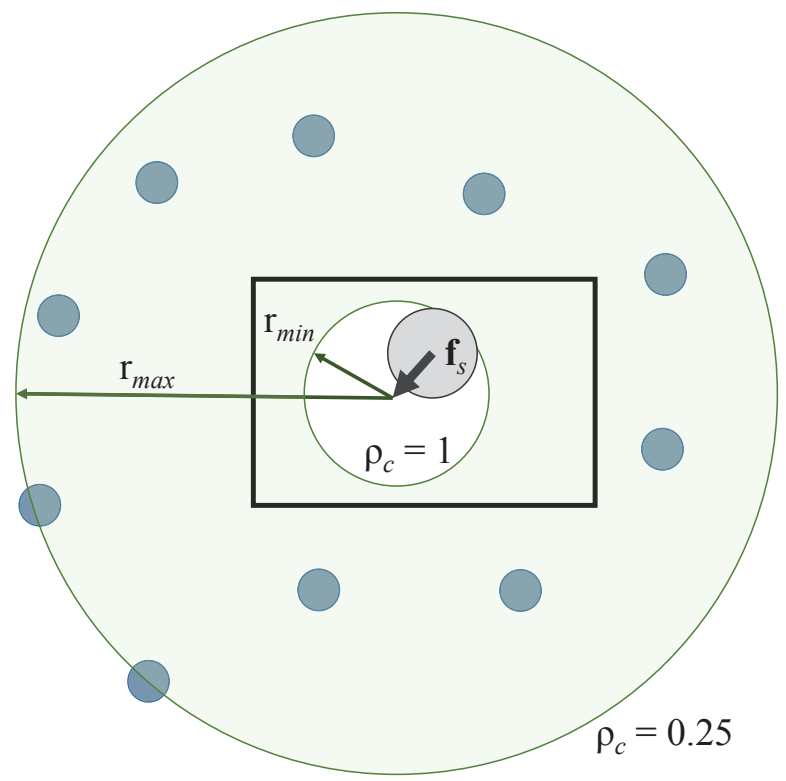

(a) Initial Uniform Spacing

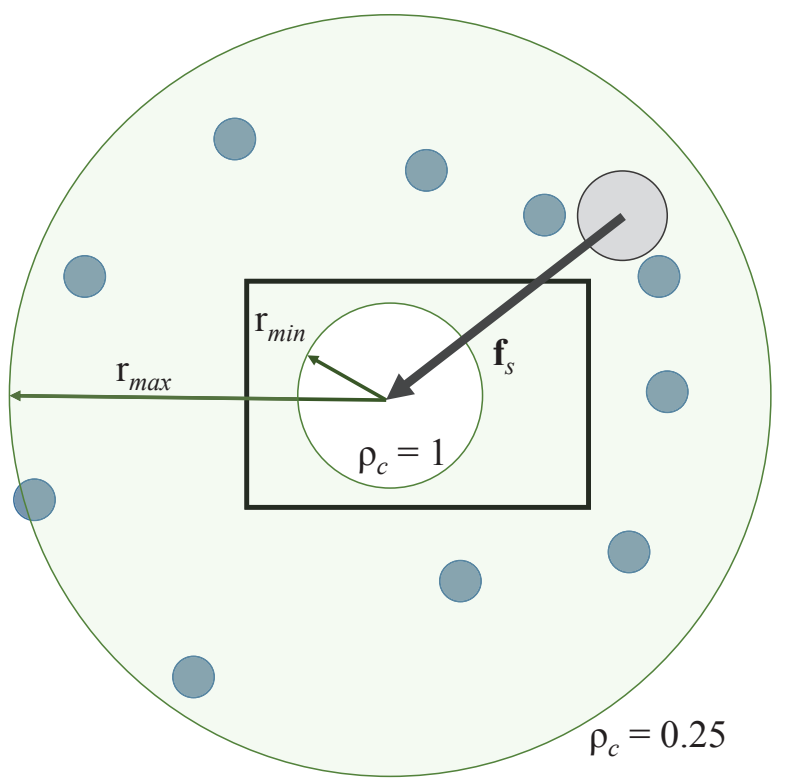

(b) Adjusted Spacing

Figure 2.6: This image shows the process of spacing manipulation, where the spacing between the robot closest to the haptic cursor and its neighbor will be smallest, governed by the value of $\rho_{c}$. The feedback force, $\mathbf{f}_{s}$, is directed toward the center of formation.

The equilibrium distance $L_{i}$ is assigned to the space between the $i$-th and the $i+1$-th robots, with the only special case being the $M$-th and 1st robots share $L_{M}$. These distances determine how the robots are distributed within the shape, irrespective of the dynamics that govern the shape in a more continuous environmental space. Nodal network properties such as total perimeter, $P_{n}$, and center of mass, $\mathbf{C O M}_{n}$ are used to adjust these distances based on cursor location. The initial equilibrium distance is $L_{0}=P_{n} / M$ and each equilibrium distance value is governed by the relationship $L_{i}=\rho_{i} L_{0}$, where $\rho_{i}$ is the equilibrium distance factor.

As the cursor moves a distance $r$ away from $\mathbf{C O M}_{n}$, the robot with closest proximity to the cursor is denoted robot $c$. The equilibrium distance factor for this robot is set first and is given by

$$
\rho_{c}= \begin{cases}1 & r<r_{\text {min }} \\ 1-0.75 \frac{r-r_{\text {min }}}{r_{\max }-r_{\min }} & r_{\text {min }} \leq r \leq r_{\text {max }} \\ 0.25 & r>r_{\max }\end{cases}
$$


where $r_{\max }$ is the distance from $\mathbf{C O M}_{n}$ to robot furthest away from $\mathbf{C O M}$ and $r_{\min }=0.25 r_{\max }$. From there, other factors are calculated as

$$
\rho_{i}=\rho_{c}+\frac{2\left(\rho_{\max }-\rho_{c}\right)}{M} n
$$

where $n$ is the minimum number of robot nodes aways from robot $c$ and $\rho_{\max }=2-\rho_{c}$. Equation (2.5) and having $\rho_{\max }$ be dependent upon $\rho_{c}$ ensure that $\sum_{i=1}^{M} L_{i}=P_{n}$, which is to be expected.

A sum of the distance between robot nodes is calculated by accumulating the distances between nodes along the nodal network and is denoted as $d_{i}$. Robot nodes transfer to neighboring nodes in order to maintain $L_{i}-l_{i} \leq d_{i} \leq L_{i}+l_{i}$, where $l_{i}=0.25 L_{i}$. In other words, if a distance between robot nodes is within $25 \%$ of the calculated equilibrium distance, then equilibrium is achieved. This tolerance allows for a range of distances to acquire equilibrium and prevent desired robot positions from switching unnecessarily between neighboring nodes.

This is analogous to a virtual spring network, where the equilibrium distances can be thought of as variable unstretched lengths and the robots are adjusting position to minimize potential energy in the system. Due to the finite amount of nodes in the network, robot node positions simply establish the desired positions of robots. The continuous dynamic motion is handled separately by applying forces to the robots and guiding them directly to these positions (See Section 2.1.2).

When this type of manipulation is enabled, a force, $\mathbf{f}_{s}$, is produced which is directed toward $\mathbf{C O M}_{n}$, proportional to the cursor's distance away from $\mathbf{C O M}_{n}$, such that

$$
\mathbf{f}_{s}=k_{s}\left(\mathbf{C O M}_{n}-\mathbf{p}_{\text {cursor }}\right)-b_{s} \mathbf{v}_{\text {cursor }}
$$

where $k_{s}$ is the spacing force stiffness coefficient, $b_{s}$ is the spacing damping coefficient, and $\mathbf{p}_{\text {cursor }}$ and $\mathbf{v}_{\text {cursor }}$ are the position and velocity of the haptic cursor, respectively. This feedback is designed to give the operator a sense of the gesture direction relative to the team by modeling the force as a virtual spring that connects the cursor to the team's center-of-mass. The magnitude also informs the operator of the strength of the spacing adjustment in that direction. As the operator may be quickly gesturing toward various sections of the team shape, a damping force is provided to prevent the device motions from being too abrupt. This damping effect also helps the device feel more like 
a grounded joystick rather than a free-moving cursor. The ground force feedback is also active in this mode, so the total feedback force felt by the operator will then be

$$
\mathbf{F}_{s}=\mathbf{f}_{g}+\mathbf{f}_{s}
$$

\subsection{Travel Interaction}

Travel interaction allows the operator to move the team from one building to another. As mentioned, sometimes the team can separate and split into two groups. Identifying the position of all robots is necessary to send additional travel commands and collect the team together again. The operator receives visual and haptic feedback to describe the positions of all robots in the team. Perhaps the most obvious is the visual feedback, where each robot is represented as a circle on a display (See Section 3.4.1). The haptic feedback is divided into relative travel and shape exploration forces, which are explained in the following sections.

\subsubsection{Relative Travel}

The relative travel force occurs while the team is traveling between buildings. It activates once the travel position is being chosen, and disactivates once the team has switched to surround mode. The relative travel force is

$$
\mathbf{f}_{r}=k_{r}\left(\mathbf{C O M}_{n}-\mathbf{p}_{\text {cursor }}\right)-b_{r} \mathbf{v}_{\text {cursor }}
$$

where $k_{r}$ is the relative travel stiffness coefficient and $b_{r}$ is the relative travel damping coefficient. Similarly to the feedback force provided during a spacing manipulation, a small level of damping is used to stabilize the cursor motion while choosing a travel position. The total haptic feedback force felt by the operator during relative travel is then

$$
\mathbf{F}_{r}=\mathbf{f}_{g}+\mathbf{f}_{r}
$$

This force is designed to help the operator gain a sense for the relative distance between the cursor and the team. The force is strong enough to guide the device toward the team location, 
but not strong enough to prevent the operator from moving the device as desired. With training, the operator may interpret these forces to gain a better sense for where and how the team is traveling. Once the operator chooses a desired travel location, the user may also experience a shape exploration force, explained in the next section. Figure 2.7 shows the process of sending a travel command to the team. The first step is choosing a location by pressing the TRAVEL button, moving the haptic cursor to a desired location and then releasing the button. Once that is done, the team has received a travel location and attempts to move to that location. The operator experiences the relative travel force from the device as the team travels. This discrete command process is used rather than continuously "dragging" the team via velocity commands so that the operator can occupy the time on more urgent tasks while relying on the autonomous behavior of the team to complete the travel motion.

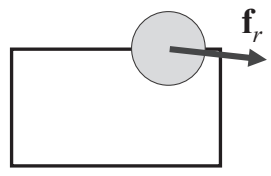

(a) Travel command in process

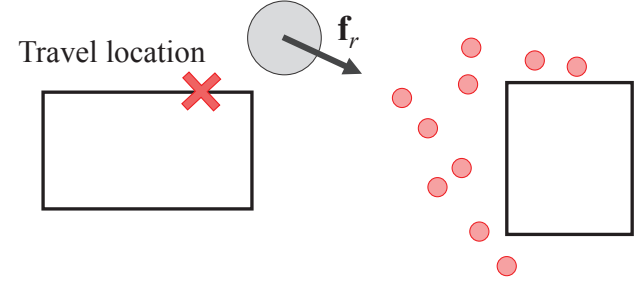

(b) Travel command is applied

Figure 2.7: This image shows the process of setting a travel command and how the relative force feedback, $\mathbf{f}_{r}$, is directed toward the team. Notice that in (a), the team is still in surround mode, so the travel location is still being chosen. In (b), the team attempts to form near the travel location (marked with a red $\mathrm{X}$ ).

\subsubsection{Shape Exploration}

After a travel command has been sent, the team works to move to the desired travel location. During this time, the operator may explore the overall shape of the team by coming in contact with force nodes in a dynamic network. The operator is not, however, given the ability to change the shape or spacing of the team while in travel mode because the purpose of the travel is simply to move to another building. 
A convex hull [42] of robot locations is computed based on their global positions in the environment. A travel-mode nodal network is formed by using the robots which are located on the edge of the convex hull as an ordered list of positions, shown in Figure 2.8. This nodal network forms the outer boundary of the overall shape of the team.

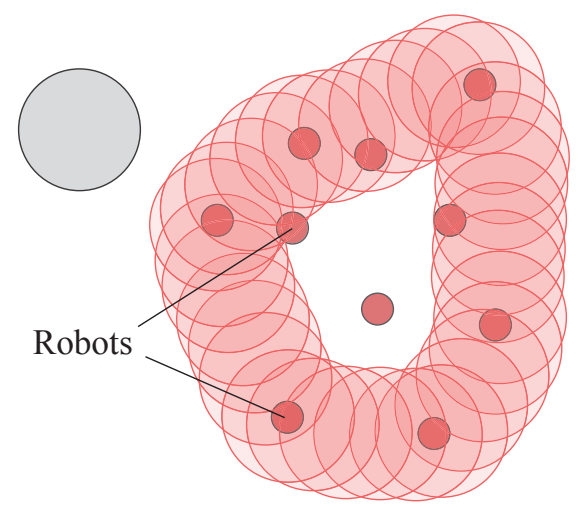

Figure 2.8: This image shows how force nodes (light red) are created from the convex hull after the team (dark red) has began to travel to another building.

Since robots are also positioned within the nodal network, a virtual surface is needed to enclose the shape formed by the nodal network. This virtual surface lies tangent with the top of the nodes and within the convex hull. When the cursor comes into contact with this surface, an additional force is produced to simulate an enclosed volume. To help visualize this force, a side-view of the nodal network is shown in Figure 2.9.

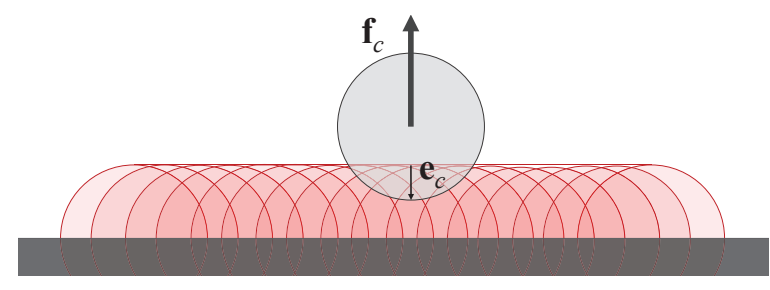

Figure 2.9: This figure shows a side-view of the force produced by contacting the surface formed by the convex hull. 
Similar to the ground force, the convex hull surface is modeled as a flat, stiff surface. The force produced when the haptic cursor comes into contact with this surface is

$$
\mathbf{f}_{c}=-k_{c} \mathbf{e}_{c}
$$

where $k_{c}$ is the convex hull stiffness coefficient and $\mathbf{e}_{c}$ is the penetration vector into the bounding surface.

The force produced by contacting the force nodes, $\mathbf{f}_{n}$ is given in Equation 2.3 and the relative travel force is also in effect, so the total haptic feedback force felt by the operator during travel shape exploration is

$$
\mathbf{F}_{t}=\mathbf{F}_{r}+\mathbf{f}_{n}+\mathbf{f}_{c}
$$

\subsection{Chapter Summary}

This chapter presented each type of interaction in surround and travel modes, along with haptic feedback provided to the operator in each of those modes. In surround mode, the operator may explore or manipulate the team shape in order to cause the robots to move outside the initial formation chosen by their autonomous behavior. The spacing between robots can also be adjusted through spacing manipulation. In travel mode, the operator may choose a desired travel direction and explore the shape of the team while moving between buildings. All of the force feedback is modeled as virtual spring-and-damper systems, using their own governing models from virtual surfaces to relative distance and velocity. Implementation of these theoretical modes with a specific haptic interface in the simulation system is discussed in Chapter 3. 


\section{CHAPTER 3. SIMULATION SYSTEM AND INTERFACE}

The simulation system was designed to experimentally determine an operator's effectiveness in controlling a small team of robots with haptic feedback, and will be referred to as the Robot Team Haptic Simulator (RoTHSim). This chapter describes the components and features of RoTHSim.

\subsection{System Overview}

RoTHSim consists of a haptic interface (Novint Falcon), a PC computer with dual monitors and software that runs the simulation, as shown in Figure 3.1. The keyboard and mouse are used as well, but only in providing additional input needed for the human-subject experiment, explained in Chapter 4. The Novint Falcon is the only controller used to manipulate and interact with the simulated robot team.

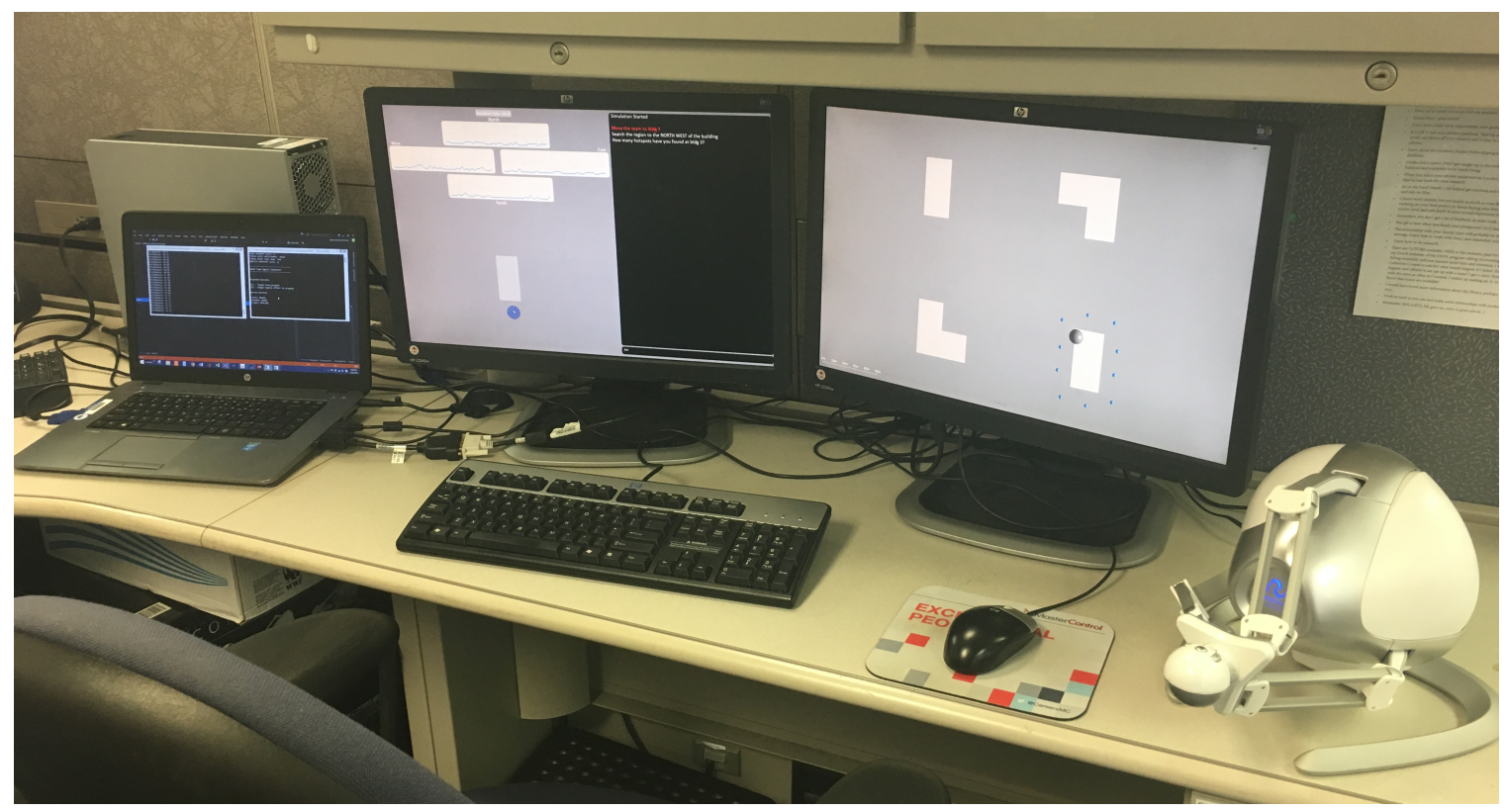

Figure 3.1: Simulation system. 
A process diagram of the RoTHSim software is presented in Figure 3.2 and shows how the software is used to simulate an operator's interaction with a robot team. The robot behaviors model, discussed in Section 2.1, is implemented as a local server running on the host computer. The RoTHSim C++ Application is the heart of the simulation, which handles communication to the server, haptic feedback calculations, and recording simulation data. This graphical user interface is viewed by the operator using two computer screens, displaying the team, its environment, the haptic cursor position and other relevant simulation information. The operator sends and receives commands to the team via the haptic interface.

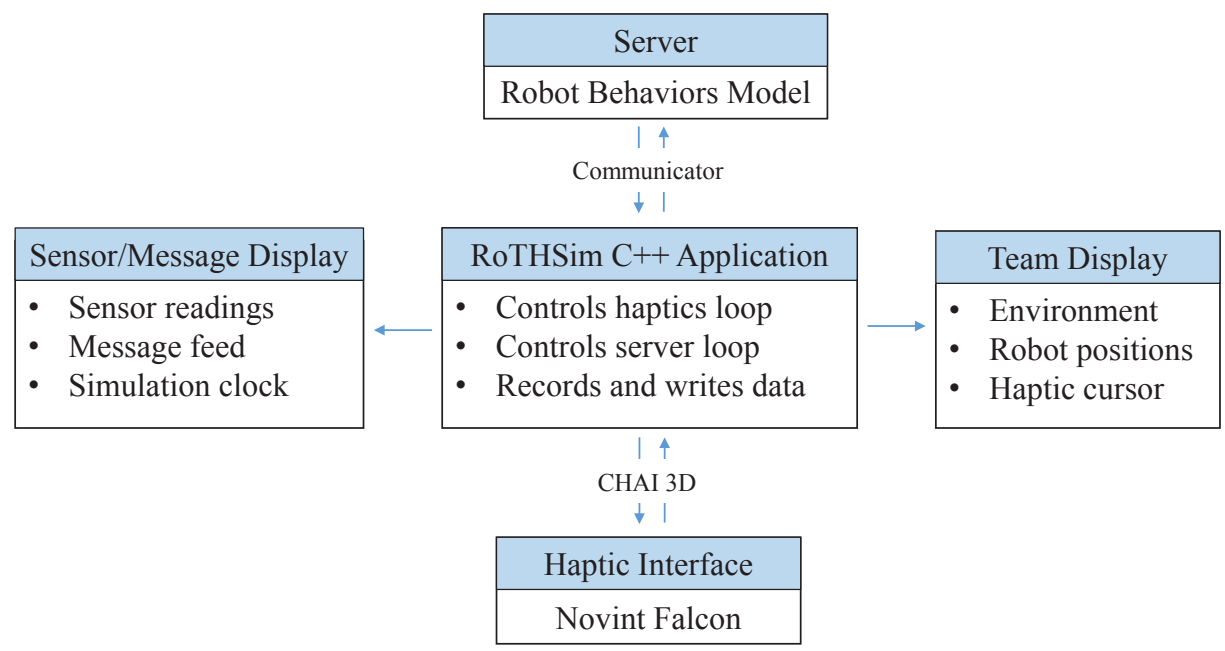

Figure 3.2: Simulation system overview.

\subsection{Novint Falcon and CHAI 3D}

The Falcon is a commercially available, USB haptic feedback device developed by Novint Technologies Inc. This device provides 3D position sensing and produces a maximum force of $8 \mathrm{~N}$ (1.8 lbf). The total workspace is $10.16 \mathrm{~cm}$ (4 in) wide in $x y z$, which is a relatively small workspace compared to other haptic devices. Since the operator is asked to concentrate on multiple tasks while using the device, this smaller workspace is an advantage by preventing the need to make large motions in order to move the team accordingly. There are a total of four buttons on the device handle, and three of them are used to signal a type of control over the team, mentioned in 
Chapter 2. Referring to Figure 3.3, and assuming a right-handed configuration, the buttons are mapped as follows:

- TRAVEL The left button (closest to thumb) is used to start and finish travel commands.

- SHAPE The middle (center) button is used to engage shape manipulation.

- SPACING The right button (closest to index finger) is used to engage spacing manipulation.

This device was also chosen due to its low-cost, making it feasible to extend control modes to include two devices instead of just one (See Section 6.3).

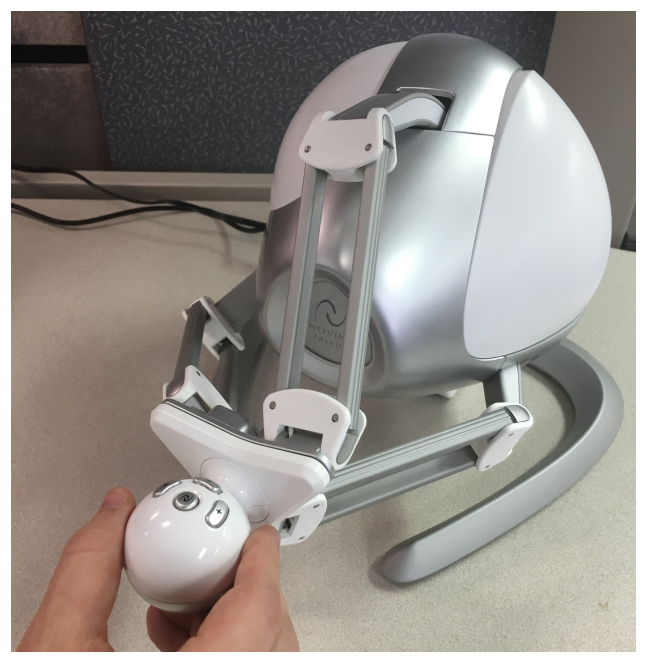

Figure 3.3: Novint Falcon.

CHAI3D is an open-source, "cross-platform C++ framework designed for computer haptics, visualization and interactive real-time simulation" [43]. The CHAI3D C++ libraries are used to connect to the Falcon, map device position to a virtual haptic cursor on a display, visualize an environment using OpenGL, and provide feedback forces to the operator.

\subsection{Robot Behaviors Server}

The robot team behavior is described in detail in Section 2.1, but this section briefly explains how the theoretical model is implemented in software. For the purposes of this research, 
the robot behavior has been delivered in the form of an executable file that runs locally on the computer of choice.

This executable will be called the "server" in this thesis, because it runs on a local network connection. The underlying code was developed using the Go programming language, so the team information, including (but not limited to) robot positions, modes, inter-robot radius, etc., is accessed by sending and receiving messages over a TCP socket, which are then encoded using the Protocol Buffer standard [44]. Additional C++ implementation was provided by Alder to accomplish this data transfer. The process of messaging to and from the server will be called the "communicator." The sent messages by the communicator to the server permit user influence over the model.

\subsection{RoTHSim C++ Application}

The RoTHSim software consists of a C++ application that controls graphics, haptics and server loops, which run in parallel throughout the simulation. The main haptics loop operates

at $1 \mathrm{kHz}$ and runs in its own thread, and is where all the haptic feedback calculations are being made. Some of the methods in the communicator require some time to complete, so to prevent the simulation from slowing down, the server loop is also placed in its own thread. This way, any delay in the communicator is noticed in the server loop and the haptic sensation is preserved. The next sections will describe additional software components that form the graphical user interface.

\subsubsection{Team Display}

One of the two displays used in RoTHSim is the team display, which shows the robot team and its environment from a bird's eye view. An example of a simple environment is shown in Figure 3.4a. The gray background represents a ground surface that is the two-dimensional plane in which the team resides. Buildings in the environment are shown as simple polygon shapes colored white. The robot team is represented as small spheres on the display and the haptic cursor is the larger sphere that maps to the device position in three dimensions. Upon closer inspection to the zoomed view in Figure 3.4b, a persistent dotted line also marks the spanning ring shape of the team. Once the team initially surrounds the building, the spanning ring shape may be obvious, but 
may becomes less so as the operator manipulates the shape. The dotted line also helps the operator identify the shape of the team without haptic feedback.

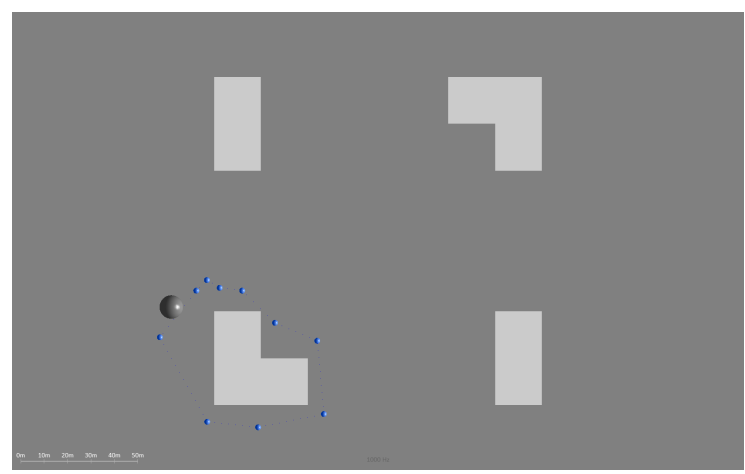

(a) Team display

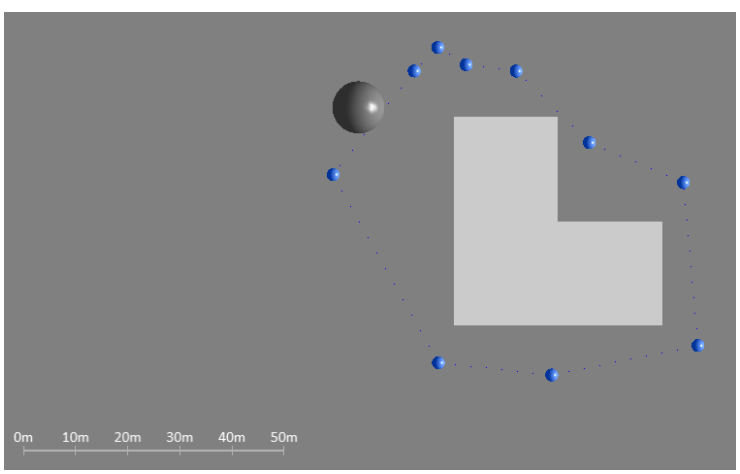

(b) Enlarged view of the team

Figure 3.4: This figure shows two views of the same team environment, consisting of the ground surface (gray), buildings (white), team (blue) and haptic cursor (dark gray). A distance scale is also shown in the bottom-left corner.

\subsubsection{Hotspots Implementation}

Hotspots represent points of interest in the environment. The operator's primary task is to search for and find hotspots. In a military scenario, these hotspots could represent locations of possible enemy targets, bombs or other threats. They could also be used to identify search paths or possible survivor sightings in search-and-rescue scenarios. Whatever the scenario, hotspots have been created to generalize points of interest during a reconnaissance or surveillance mission. To simulate urgency in locating hotspots, each hotspot is only active for a certain window of time, with a maximum of 40 seconds. Hotspots are hidden from the operator's view until they are found or expire. When found, they show on the display as green squares until they have reached an expiration time, in which they fade away from view (See Figure 3.5a). When they expire and have not been found, they show on the display as red squares for a brief moment and fade away from view (See Figure 3.5b). In a realistic scenario, an operator will not be able to view what has not been uncovered (as is the case with showing expired hotspots), but this feature has been included for the purposes of a human subject experiment (See Chapter 4). 


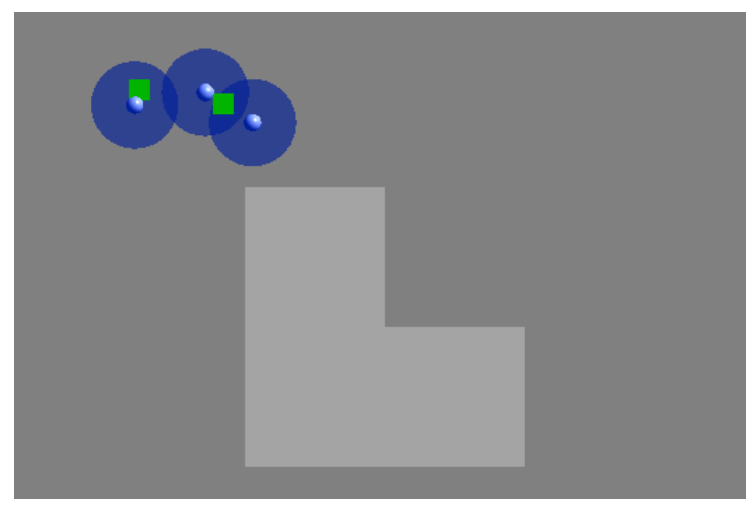

(a) Hotspots have been found

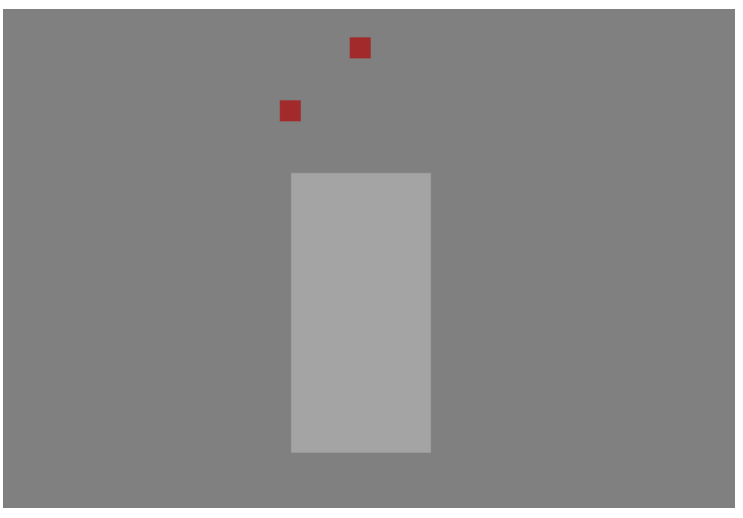

(b) Hotspots have expired

Figure 3.5: This figure shows how found or expired hotspots are visually displayed. In (a), the robots (light blue spheres) are within sensing range (dark blue circles) of the hotspots (green squares). In (b), robots have not been in sensing range long enough (if at all) to find the hotspots (red squares), causing them to expire before being found.

Hotspots are detected by means of a sensor attached to the center of each robot. Each sensor reading is binary, in that it has only two possible outcomes, detection or no detection. A true positive detection occurs when a reading is obtained while a hotspot is within sensor range. The true positive rate, $p_{t}$, is modeled probabilistically with a Gaussian distribution from the location of the sensor, given by

$$
p_{t}=p_{\min }+\left(p_{\max }-p_{\min }\right) e^{-d^{2} / \sigma^{2}},
$$

where $p_{\min }$ is a minimum true positive rate, $p_{\max }$ is a maximum true positive rate, $d$ is the euclidean distance from the sensor and $\sigma$ is the standard deviation of the Gaussian curve. With $r_{\text {sensor }}$ being the range of the sensor, the standard deviation was chose to be $\sigma=r_{\text {sensor }} / 3$. Sensor readings are not always indicative of nearby hotspots. A false positive detection occurs when a reading is obtained while no hotspots are in range. The false positive rate, $p_{f}$, is another predetermined constant. Values given to the mentioned constants are $p_{\min }=0.50, p_{\max }=0.80$, and $p_{f}=0.10$.

\subsubsection{Sensor/Message Display}

The second display (Figure 3.6a) shows the surrounded building and the sensor locations where there have been either true or false detections. A sensor reading is shown on the display as a small sphere (representing the robot) and a circle with radius $r_{\text {sensor }}$. The reading initially flashes 
visible and fades away after a short amount of time, unless another reading is made. If a robot is continuously near a hotspot, the sensor will flash repeatedly or appear to remain lit most of the time, depending on the robot's proximity to the hotspot. Sensor readings are also encoded into probabilities of detection in the four cardinal directions around the building. A history of these probabilities is shown through time on four running plots.

This display also shows incoming messages that have been preprogrammed to appear at certain times in the simulation. The operator may also type messages and they are saved in the incoming message feed.

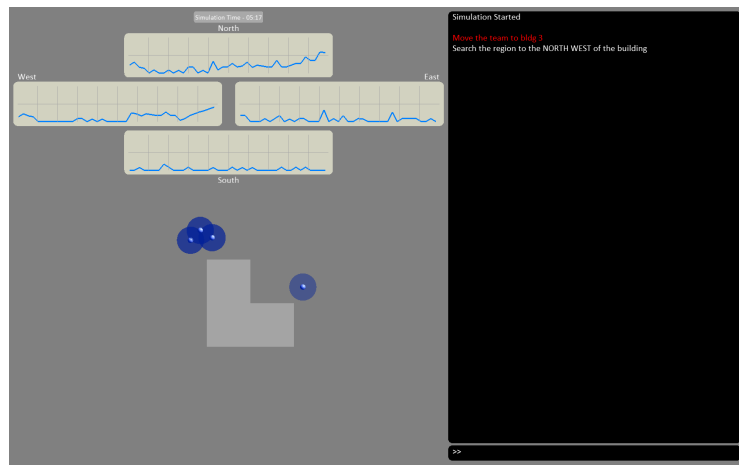

(a) Sensor/Message display

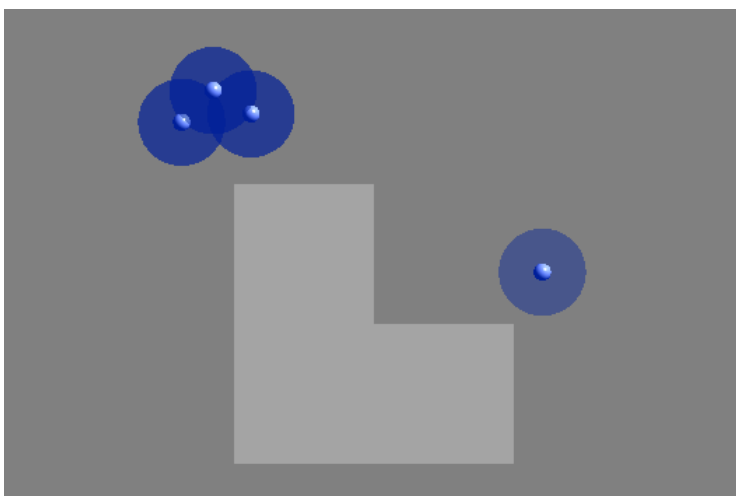

(b) Enlarged view of the sensor readings

Figure 3.6: This figure shows two views of the sensor and message display while detecting hotspots in surround mode. The persistence of readings to the northwest of the building is indicative of hotspots in that area.

\subsubsection{Data Manager}

The simulation time, positions of each robot, team mode (surround or travel), haptic cursor position and the haptic feedback force are recorded and saved to a text file for post-processing. To prevent overly frequent commands of writing to a file, data from each iteration is pushed into a list and written at a rate of $10 \mathrm{~Hz}$. All user events are recorded, including the button presses of the haptic device and any user input from the keyboard. When hotspots are found, their identification number and the time at which they are found are also recorded. 


\subsection{Tutorial}

An interactive tutorial was developed to teach the components of RoTHSim to the operator. Throughout the tutorial, portions of the screen are highlighted and instructions are given on screen. Example screenshots during the tutorial are shown in Figure 3.7. The entire tutorial takes between 20-25 minutes to complete, providing a new operator with a reasonable amount of time to become acquainted with the user interface and capabilities of the robot team.

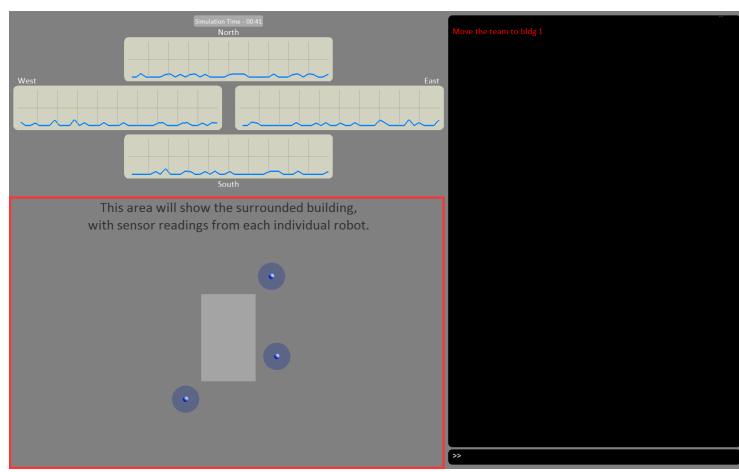

(a) Sensor reading instruction

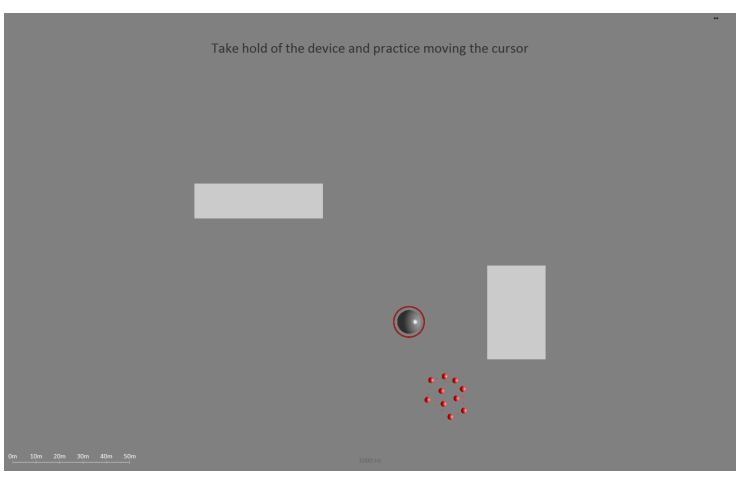

(b) Haptic cursor instruction

Figure 3.7: This figure contains a view from each of the displays at two different times during the tutorial. In (a), the operator learns how to interpret the sensor readings. In (b), the operator learns how to map device movement to cursor movement.

\subsection{Chapter Summary}

This chapter described the various components of the Robot Team Haptic Simulator, RoTHSim. RoTHSim is an experimental platform used to determine the effectiveness of an operator's heads-up control of a robot team. The use of RoTHSim and a description of the human-subject experiment is found in Chapter 4. Possible improvements to RoTHSim are listed in Chapter 6. 


\section{CHAPTER 4. HUMAN SUBJECT EXPERIMENT}

A human subject experiment was conducted to examine the effects of haptic feedback on an operator's ability to control a robot team while performing several tasks. This chapter describes the purpose of the study, explains the experiment design, and provides insight into methods of measurement.

\subsection{Purpose}

Past research involving human-robot interaction has shown interest in military-based scenarios that require a single operator to teleoperate one or more robots while performing other mission-related tasks [45]. In these scenarios, multitasking often has a negative impact on operator performance, workload and SA [28]. Naturally, research has aimed to mitigate these losses.

A common approach is to provide the operator with a multimodal interface, which helps to divide task stimuli amongst several senses. Supplementing visual feedback with tactile or audio feedback can then be thought of as providing additional resources that can be used to the operator's advantage when vision alone is insufficient. In work done by Chen \& Terrance [46], an operator was notified of potential targets near a mounted vehicle with tactile cues. Results from that study showed that multitasking performance was significantly improved with the use of tactile feedback. In a more recent study by Fields et al. [26], an operator was asked to control a swarm of UAVs as they follow a convoy and report on swarm health and communication status. Tactile cues were also used in that study to assist the operator in reducing response time.

The objective of this research is to investigate the effects of haptic feedback on an operator's ability to manage a team of robots in reconnaissance and surveillance tasks. Specifically, it is of interest to determine how well an operator can maintain heads-up control of the team while being asked to multitask. The main effects of interest are percent hotspots found, situation awareness and workload. It is hypothesized that haptic feedback will increase the operator's primary task 
performance (percent hotspots found), improve situation awareness and decrease the operator's workload.

\subsection{Experimental Apparatus}

The experiment was held in the MAGICC Lab on Brigham Young University (BYU) campus. Each subject used a dual-monitor, multimodal workstation as shown in Figure 4.1. The PC computer used to run the simulation is a Hewlett-Packard Elite 850 Series laptop with Intel i7 processor, 16 GB of RAM and an integrated Intel HD graphics card. Two monitors were used with a 32-bit color resolution of 1920 x 1680 pixels. The Novint Falcon desktop haptic device was placed to the right of the monitors and is the primary controller used during the simulation. A standard keyboard and mouse were also included, which are used infrequently throughout the experiment. The operator uses their dominant hand to control the Falcon and non-dominant hand to enter keyboard input.

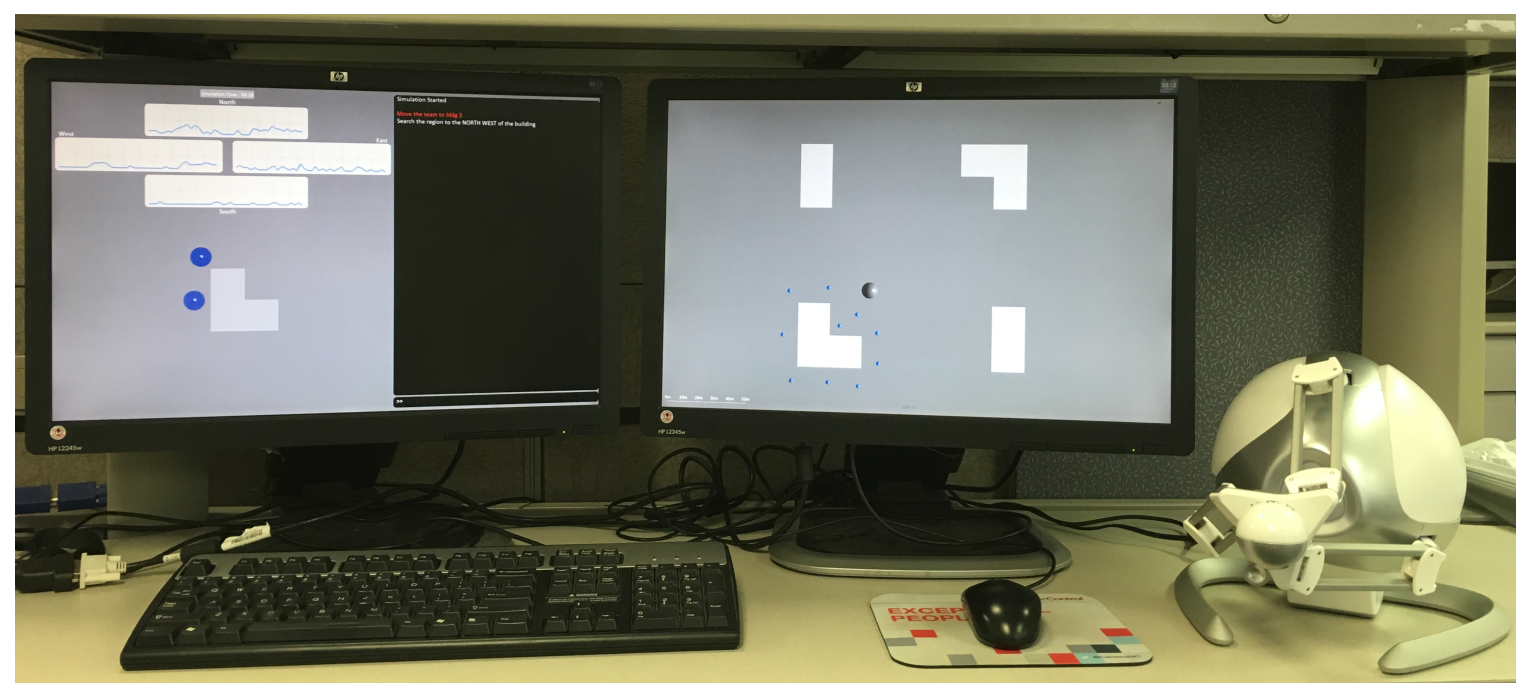

Figure 4.1: Dual-monitor, multimodal workstation.

\subsection{Experimental Approach}

RoTHSim was designed as a means to experimentally determine how well an operator can manage a team of robots while being required to perform additional mission-related tasks and 
switch attention between multiple displays. The following sections will explain the approach to the experiment to better understand how RoTHSim is used.

\subsubsection{Task Description}

When using RoTHSim, an operator has two main tasks, which are:

1. Primary task: Continuously search for and find as many hotspots as possible.

2. Secondary task: Respond to and follow incoming messages.

The primary task involves manipulating the team and creating various shape and spacing combinations in order to maximize the number of hotspots being uncovered. As explained earlier, hotspots are points of interest in the environment. The operator is only concerned with locating and uncovering hidden hotspots and not in interpreting their meaning. Past research has used hidden points in an environment as a means of measuring team coverage [16]. They will be used in this study to simulate a reconnaissance task. The operator does not search blindly, but is provided a sensor reading for each robot. The strength and location of the sensor readings in the environment are used to deduce possible locations of hotspots. Hotspots will be hidden in groups of one, two or three, which all need to be detected within a short window of time for the group to be counted as found. This requires the operator to make a specific formation in order to detect and find all the hotspots in each group. Placing hotspots in clustered groups that span various paths is used to indirectly determine an operator's effectiveness in manipulating the team. For each environment, the percentage of hotspots found is used as the main measure for primary task performance.

The secondary task involves focusing on the incoming message feed, which has been preprogrammed to display specific messages that are timed with the simulation. The operator is asked to respond to incoming messages, which include:

- Relocation instructions These messages are mandatory instructions that always come in the form "Move the team to building \#."

- Search suggestions These messages ask the operator to use shape or spacing manipulation to search specific regions around a surrounded building. They will always help the operator in finding hotspots and are not meant to be misleading. 
- SA questions These messages are probing questions which are used to measure the operator's SA. A description of these questions is given in Section 4.3.4.

The operator responds to relocation instructions and search suggestions by manipulating the team accordingly. A typed numerical response is required to respond to SA questions.

\subsubsection{Multitasking and Heads-up Awareness}

Given a single viewpoint of an environment, while it is most convenient and realistic to concentrate all visual information on a single display, RoTHSim splits information across multiple displays in order to simulate a less than ideal case. For example, robot state information is shown on the main team display, but sensor readings are shown on a second display. Although the user may desire those two pieces of information to be visually displayed on the same screen, they are separated to simulate a situation in which the operator needs to be aware of surroundings rather than being heads-down in the team display. This is metaphorically similar to using intermittent algorithmic problems as a means to simulate occasional multitasking. In a practical teleoperation scenario, unlike this experimental scenario, additional events that may require the operator's attention may include video surveillance, mission planning, mission-related discussions with other people or interruptions due to emergency. Since many of these more realistic scenarios require extensive training, a simple division of information was preferred and remains ecologically viable.

It has been posited that the presence of haptic feedback will have a positive effect on an operator's performance while placed in this multitasking scenario. Therefore, experimental comparisons need to be made to test this claim. In situations where task demand is low, it is expected to see little difference between measurements between the presence and absence of haptic feedback. When more task demand is placed on the operator, it is expected that task performance will decrease, SA may decrease and workload may increase as compared to when task demand is lower. If haptic feedback has an influence on the experimental measures, there should be a noticeable difference between the presence and absence of haptic feedback while observing results from environments with high or low task demand. The level of task stimuli will be referred to as world difficulty and is discussed in Section 4.3.3. 


\subsubsection{World Difficulty Classification}

The world difficulty is determined by the level of primary task difficulty. Two levels of world difficulty, easy and hard, are used in this experiment. In each environment, there are hotspots hidden in groups around several buildings. The operator is told which building to search at specific times during the simulation, so difficulty is not based on determining which building to travel to. World difficulty is calculated as the sum of each hotspot group difficulty in that world. Each hotspot group difficulty is

$$
d_{h}=p_{h}(H-1)+p_{d}\left(1-\frac{t_{h}-\tau_{\min }}{\tau_{\max }-\tau_{\min }}\right)
$$

where $p_{h}$ is a group size factor, $H$ is the number of hotspots in the group, $p_{d}$ is an active duration factor, $t_{h}$ is the group active duration, $\tau_{\min }$ is the minimum active duration and $\tau_{\max }$ is the maximum active duration. Calculating group difficulty in this way models larger groups as much more difficult to find, which is accurate based on subjective observations of attempting to find hidden hotspot groups of different types. Equation 4.1 does not give an absolute measure of difficulty, but it does

provide a way to ensure that easy or hard worlds are classified appropriately. For example, easy worlds will have a lower difficulty score than hard worlds. The difference between easy and hard difficulty levels is arbitrary and needs to be tuned based on the experiment, as will be discussed in Section 4.5.2.

\subsubsection{Situation Awareness Measurement}

As mentioned in Section 1.1.3, there are many proposed methods of objectively or subjectively measuring SA. A different method than those discussed is proposed in this experiment.

Hone et al. [32] used the three main components of Endsley's definition of SA (perception, comprehension, projection) of what can be termed "awareness," to construct a set of questions used to measure SA, called the 3-Q Model. This model was developed with the purpose of creating a foundation model of SA that can be applied to combat situations, air traffic control, or even sports [32]. The three types of questions are: 
- WHO IS WHERE? - This suggests a perception of elements in the environment, either animate or inanimate.

- WHAT ARE THEY DOING? - This suggests a comprehension of what purpose or role each of the elements are playing in the environment.

- WHAT WILL THEY DO? - This suggests a projection or prediction of how those elements will behave or be acted upon at some point in the future.

This model provides a way to operationalize Endsley's definition and reduce the whole of SA into simple components. To measure SA, one does not simply ask these three questions to the operator. Rather, they are used as a framework to develop specific questions that test an operator's awareness of the experimental scenario.

In the present experiment, the operator is told to keep a mental model of the expired hotspots throughout the environment, whether they had been found or remained hidden. A set of questions that follow the 3-Q model were created and the format of these questions are:

- How many hotspots have you found at building \#?

- What percent of found hotspots where located to the (direction) of building \#? The direction is chosen from any of the cardinal directions.

- Where are you most likely to find hotspots at building \# in the future?

For the first two types of questions, the operator is only to consider those hotspots that have been found and responds by typing a number on the keyboard. The third question asks the operator to consider any hotspots that have expired, either found or hidden, and responds by placing the haptic cursor at a location in the environment and pressing ENTER on the keyboard. In each environment, hotspots are intentionally clustered together around buildings, and not proportionally distributed. The operator is told to assume that where hotspots have appeared in the past is a good predictor to where new hotspots will appear in the future. In each experiment session, each type of question will appear twice, so the operator will response to a total of six questions.

The raw numerical responses to these questions must be formed into a score in order to create an objective measure. As has been done in other objective measures of SA, there needs 
to be a comparison between the real and perceived situation [29]. To measure how quickly the operator responds to a question, the time between when the question was asked and when the operator responds was recorded as that question's response time, $t_{r}$. For each question, a response time score is calculated as

$$
s_{r t}=100\left(t_{\max }-t_{r}\right) / t_{\max }
$$

where $t_{\max }=30$ seconds. To measure the response accuracy, the deviation of the response from the correct answer is scored as

$$
s_{r a}= \begin{cases}100 \frac{\left(v_{\max }-\left|v_{c}-v_{r}\right|\right)}{v_{\max }} & \left|v_{c}-v_{r}\right| \leq 0 \\ 0 & \left|v_{c}-v_{r}\right|>0\end{cases}
$$

where $v_{\max }$ is the maximum allowable deviation value, $v_{c}$ is the correct response value, and $v_{r}$ is the operator response value. For SA questions type 1-2, $v_{r}$ is the numerical value that the operator entered. Type 1 questions use $v_{\max }=4$ hotspots and type 2 questions use $v_{\max }=40 \%$. For type 3 SA questions, this same scoring procedure is used to calculate how close the responded location, $\mathbf{p}_{r}$, was to a correct location, $\mathbf{p}_{c}$. The correct location was set to the center of mass of hotspots at the building in question, $v_{r}=\left|\mathbf{p}_{c}-\mathbf{p}_{r}\right|$, and $v_{\max }=40$ meters. Thus, more points are awarded to the operator for each question when the response is more accurate. Scores for both response accuracy and delay are averaged together to form a total SA score.

\subsection{Pilot Study}

Before conducting the human subject experiment, a pilot study was done to tune world difficulty and feedback parameters, debug the system, verify measurement techniques and project a subject sample size needed to obtain significant results. The outcomes of the pilot study were useful in developing a meaningful human subject experiment.

Seven students from BYU's MAGICC lab participated in the pilot study. The study was a balanced two-factor experiment, where haptic feedback and world difficulty were the factors. For haptic feedback the levels were haptic feedback and no haptic feedback. For world difficulty the levels were easy and hard. Each participant was given time to learn how to use RoTHSim by means of the interactive tutorial and two easy practice sessions. After the training had finished, 
each participant was told that four worlds of various difficulty would be presented in random order, and that a notification would be given prior to starting each session if haptic feedback was enabled or disabled. The sessions were timed and lasted 8 minutes long. During each session, participants were asked to manage a team of 10 robots to perform the primary and secondary tasks explained to them. Each subject reported NASA TLX workload ratings after each run, and weights were recorded at the completion of all four sessions.

Comparing the effects of world difficulty alone on primary task performance, the hard world shows a large decrease in performance, which validates the method for classifying world difficulty. Results from the pilot study suggest that the presence of haptic feedback does help to increase primary task in the hard world. This suggests that haptic feedback provides an advantage to the operator when experiencing higher task demand. Measures for secondary task performance and SA score seem to decrease in the easy world with haptic feedback, but there is little difference in the hard world. The significance of these measures can only be determined by using a larger sample size. Several observations were made to improve the design of the user study, which are listed as follows:

- Upon questioning the participants after all sessions were completed, most felt that the tasks were too difficult to complete. This was caused by too many incoming messages and suggestions. They were overly distracting and caused participants to score lower than expected. The number of incoming commands was reduced to a more manageable scale, to help participants feel more successful while performing the tasks.

- The magnitude of haptic feedback was reduced, after many participants felt like it was preventing them from moving the device as desired.

- The amount of time spent on each session was decreased from 8 minutes to 6 minutes because participants took longer than expected to submit workload ratings between sessions. This kept the total experiment time between 55 and 60 minutes.

- Tutorial sections were modified to improve how the interpretation of sensor readings was taught to the operator. 


\subsection{Experimental Design}

This section explains the components of the experimental design, including the factors and levels, world descriptions, participants and procedure.

\subsubsection{Factors and Levels}

The human subject experiment, similar to the pilot study, was designed as a full twofactorial experiment with the following factors and levels:

- Factor 1: Haptic feedback

- Levels: no haptics, haptics

- Factor 2: World Difficulty

- Levels: easy, hard

\subsubsection{World Description}

Two easy worlds and two hard worlds were developed to accommodate all the combinations of factors and levels. Thus, each difficulty level had a type 1 and type 2 world. If the same easy or hard world was used to compare haptic to no haptics, the operator will likely perform better the second time seeing the world, regardless of haptic feedback presence. The best option was to create two worlds of similar difficulty for each level of world difficulty. The combinations of factors and levels were balanced by world type to prevent one world type from always being paired with haptics or no haptics. Results from the pilot study validate this approach.

Easy and hard worlds were constructed differently, and then difficulty was compared between worlds to clearly identify them as easy or hard. Easy worlds were developed by placing hotspots in groups of one or two hotspots around three separate buildings in the environment. One building was designed to never be visited by the team. There were eleven individual hotspots and six groups of two hotspots, making a total of 23 hotspots hidden. The minimum and maximum

durations of hotspots were 15 and 40 seconds, respectively. Easy world 1 resulted in a difficulty level of 183 and easy world 2 resulted in 180, as calculated using Equation 4.1. 
Hard worlds were developed by placing hotspots in groups of one, two or three hotspots around four buildings in the environment. One building was repeated in each world, leaving one building unvisited as in the easy worlds. Eight individual hotspots, nine groups of two hotspots, and three groups of three hotspots were created, making a total of 35 hotspots hidden. The minimum and maximum durations of hotspots were 10 and 30 seconds, respectively. Hard world 1 resulted in a difficulty level of 461 and hard world 2 resulted in 470. The values of these difficulty ratings are, of course, arbitrary, but are useful in comparing the two types of worlds together to ensure that they are of similar difficulty.

Each world, easy or hard, contained four buildings in a square pattern. The buildings themselves were different shapes, mostly to provide variety in what the operator was viewing on the team display. The building shape had little to no effect on the difficulty in completing the tasks. The easy and hard world building setup is shown in Figure 4.2.

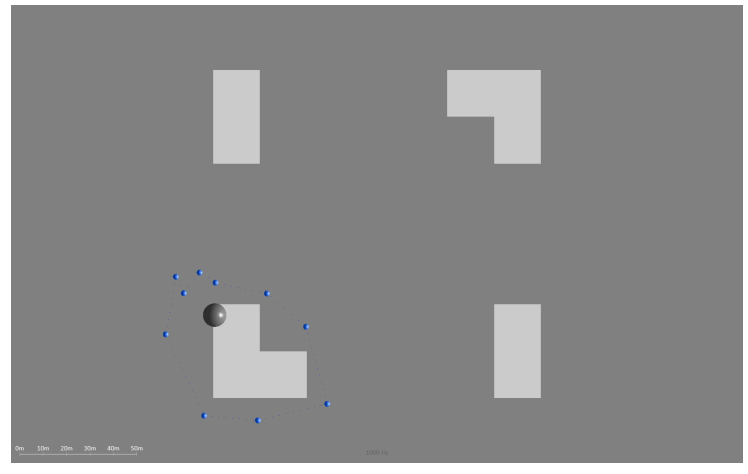

(a) Easy world

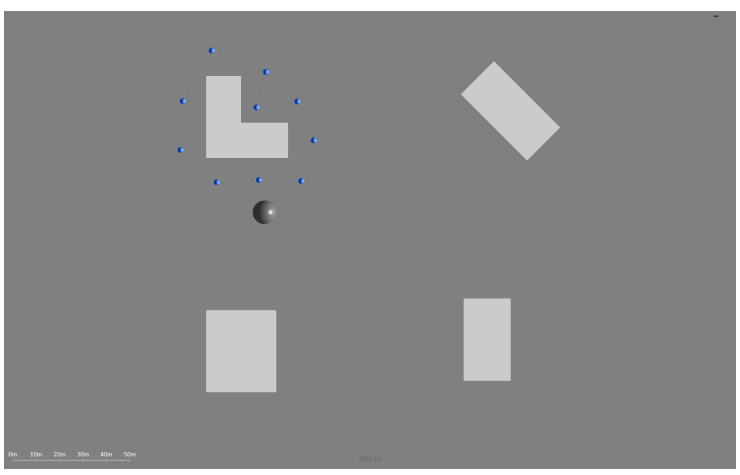

(b) Hard world

Figure 4.2: This figure shows the easy (a) and hard (b) worlds that were developed for the experiment, as seen by the operator. For simplicity, the many hotspots that are hidden in groups around each building are not shown.

\subsubsection{Haptic Parameters}

The haptic parameters, including stiffness and damping coefficients, were chosen based on intuition and operator experience from the pilot study. Each of these parameters are used in the haptic feedback equations presented in Chapter 2. They were tuned to display feedback forces 
of reasonable magnitude to the operator while still allowing the operator to move the device as desired. A summary of the values chosen is given in Table 4.1.

Table 4.1: Haptic feedback parameters, consisting of stiffness and damping coefficients.

\begin{tabular}{|c|c|}
\hline Parameter & Value \\
\hline \hline$k_{g}$ & $1500 \mathrm{~N} / \mathrm{m}$ \\
\hline$k_{n}$ & $375 \mathrm{~N} / \mathrm{m}$ \\
\hline$k_{s}$ & $200 \mathrm{~N} / \mathrm{m}$ \\
\hline$b_{s}$ & $10 \mathrm{Ns} / \mathrm{m}$ \\
\hline$k_{r}$ & $120 \mathrm{~N} / \mathrm{m}$ \\
\hline$b_{r}$ & $2 \mathrm{Ns} / \mathrm{m}$ \\
\hline$k_{c}$ & $100 \mathrm{~N} / \mathrm{m}$ \\
\hline
\end{tabular}

\subsubsection{Participants}

Based on preliminary data gathered from the pilot study and working with the BYU Center for Collaborative Research and Statistical Consulting, it was estimated that sixteen participants were needed to acquire statistically significant results for primary task performance, secondary task performance, SA and workload scores, if any exist. There were a total of 19 participants in the study, of which three were female. The ages of participants ranged from 21 to 30, with an average age of 25. Participants in this study were all students that came from a variety of majors, including Mechanical, Electrical or Computer Engineering, Computer Science, Family Sciences and Geology. Each participant was right handed and was not color-blind. Prior to starting the experiment, participants gave a self-rating on their level of video gaming experience from 0 to 10 , 0 meaning little experience and 10 meaning a great deal of experience. Those ratings ranged from 0 to 10 with an average of 5.3. There was no monetary compensation provided upon completion of this experiment and each participant was given instruction that participation in this study was strictly voluntary. 


\subsubsection{Procedure}

This experiment was performed with approval from BYU's Institutional Review Board (IRB) for Human Subjects. Prior to participation in the study, each subject was given enough time to review and sign a consent form. Although the risks were minimal, subjects were still given instruction that they could walk away from the experiment at any time if they felt any discomfort. The purpose of the experiment was explained in general terms and subjects were ensured that they were not required to have any previously knowledge of human-robot interaction to successfully complete the study. Each subject was seated in front of the multimodal workstation shown in Figure 4.1. The subject took about thirty minutes to complete the tutorial and two practice sessions with and without haptic feedback. Up to that point, the subject could ask any questions about the haptic controller, RoTHSim or the task description. The remaining four sessions were each six minutes long and were used to collect experimental data. A psuedo-random ordering of the combinations of factors and levels were used to eliminate the effects of ordering in the data. During each session, the subject managed a team of 10 robots to perform the required tasks. Upon completion of each session, the subject reported NASA TLX workload ratings. Workload weightings were reported after all sessions had been completed. Before leaving, each subject also completed an exit questionnaire to determine the subject's preference toward haptic feedback. Each subject was asked to rate their preference between 1 (not preferred) and 5 (preferred) on the use of haptic feedback while searching (SHAPE), adjusting spacing (SPACING), traveling (TRAVEL) and overall preference.

\subsection{Measures}

To determine the effectiveness of haptic feedback in the experiment, many measures were considered prior to running statistical analysis. There are primary measures, which are of most interest, and secondary measures, which help to acquire additional and more specific insight into an operator's performance.

The primary measures are:

- Found Hotspots Percentage: The percentage of hotspots found out of the total number of hotspots hidden. Since the operator is required to find as many hotspots as possible and the 
level of difficulty of the world is largely determined by the number of hidden hotspots, this is a reasonable measure for primary task performance.

- Total Command Score: This measures how well the operator responds to both team commands and SA questions. This is a weighted average of team command response scores and SA scores and determines the operator's secondary task performance.

- SA Score: An average of SA Accuracy and SA Response Scores, as explained in Section 4.3.4.

- NASA TLX Workload Score: The subjective operator workload calculated using the NASA TLX.

As a means to determine how quickly and efficiently the operator is finding hotspots, a model was developed that identifies key events during the lifetime of a hotspot. Each hotspot duration is defined by a start and end time, so those act as two of the event times. Another event occurs when a robot comes within range of the hotspot, so that it can potentially show a sensor reading to the operator. The last event occurs when the hotspot is found. These events times are shown in Figure 4.3. By using these event times, search time and manipulation time can be defined. Search time is defined as the difference between in-range and start event times. Manipulation time is defined as the difference between found and in-range event times. In the case of hotspots that remain hidden until they expire, the found event time is set to the same value as the end event time and manipulation time is calculated the same way.

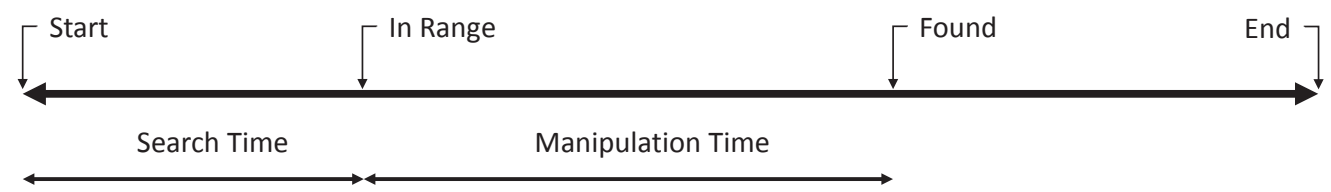

Figure 4.3: Events throughout a hotspot's active duration along a timeline. These include: start, end, in-range and found event times. Search and manipulation times are also visualized.

The secondary measures are:

- Shape Time Percentage: The percentage of total simulation time that the SHAPE button was engaged. This is used to measure the operator's dependence on shape manipulation. 
- Percent Hotspots Found by SHAPE: The percentage of hotspots found by using shape manipulation out of the total number of hotspots hidden. A hotspot is identified this way if the SHAPE button is or has been most recently engaged when it is found. Any others are identified as found by SPACING.

- Shape Hotspots Found Rate: The number of hotspots found by SHAPE divided by the total time spent manipulating the team by engaging the SHAPE button.

- Spacing Time Percentage: The percentage of total simulation time that the SPACING button was engaged. This is used to measure the operator's dependence on spacing manipulation.

- Spacing Hotspots Found Rate: The number of hotspots found by SPACING divided by the total time spent manipulating the team by engaging the SPACING button.

- Hotspots Found Rate: The number of hotspots found divided by the total time spent manipulating the team by engaging the SHAPE or SPACING buttons.

- Avg. Search Time: The average time between when a hotspot becomes activated to when it first comes in range.

- Avg. Manipulation Time: The average time between when a hotspot first comes into range to when it was found.

- Avg. Team Command Response Time: The average time taken to respond to incoming commands to manipulate the team either by shape or spacing, or travel to another building. It is used to determine how quickly the operator is responding to incoming commands pertaining to team manipulation. This is calculated similar to SA response time, except the response event occurs when the operator uses the team to search in the specified area, rather than responding via keyboard input.

- Avg. SA Response Time: The average time between when an SA question was given and when it was responded to by the operator, regardless of response accuracy.

- SA Accuracy Score: This measures how accurately the operator has responded to SA questions, regardless of response time, as described in Section 4.3.4. 


\subsection{Data Generator}

The Data Generator is a separate MATLAB application used to create world definitions by placing hotspots in groups throughout the environment and setting start and end times for each. A playback option is also included so that a designer can quickly view when and where hotspots are activated throughout the entire simulation without needing to run RoTHSim. Hotspots can be created or deleted from lists that correspond to each building. Once this data is generated, it can be loaded from or saved to a RoTHSim input file format. An example screenshot of this application is shown in Figure 4.4.

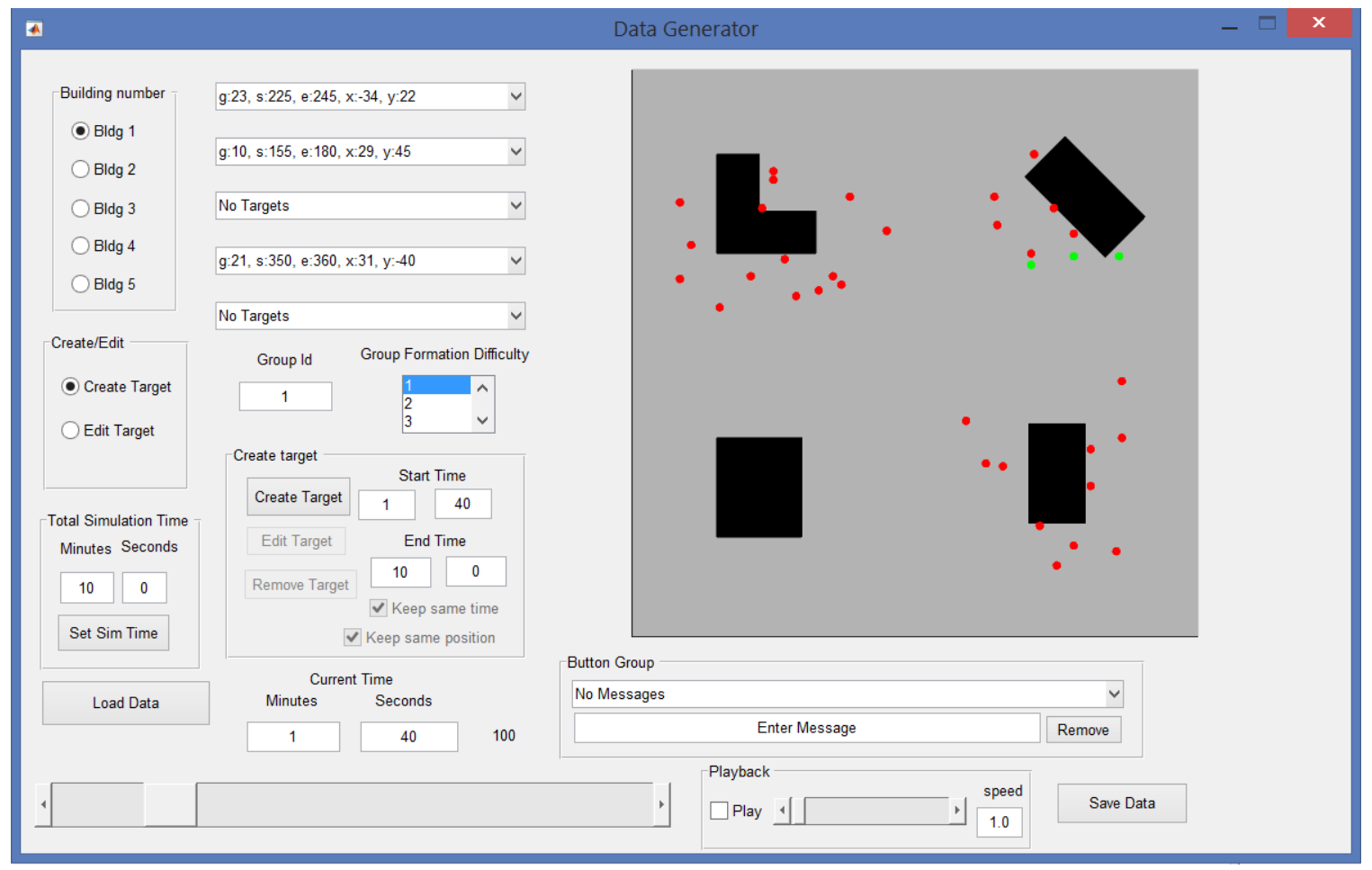

Figure 4.4: The Data Generator enables a designer to view the world and place hotspots in groups around buildings.

\subsection{Data Viewer}

The Data Viewer is another MATLAB application used to visualize each subject's data and extract experimental measures, explained in Section 4.6. The GUI (see Figure 4.5) has several components which allow viewing of robot positions, haptic cursor position, and found hotspots 
throughout an entire session on the environment plot. Responses to each SA question can be viewed with ease to determine how well the operator responded in different combinations of factors and levels. The operator's button presses throughout the entire session are also available to view. This application is also used to perform batch processing of multiple subjects and prepare data for statistical analysis.

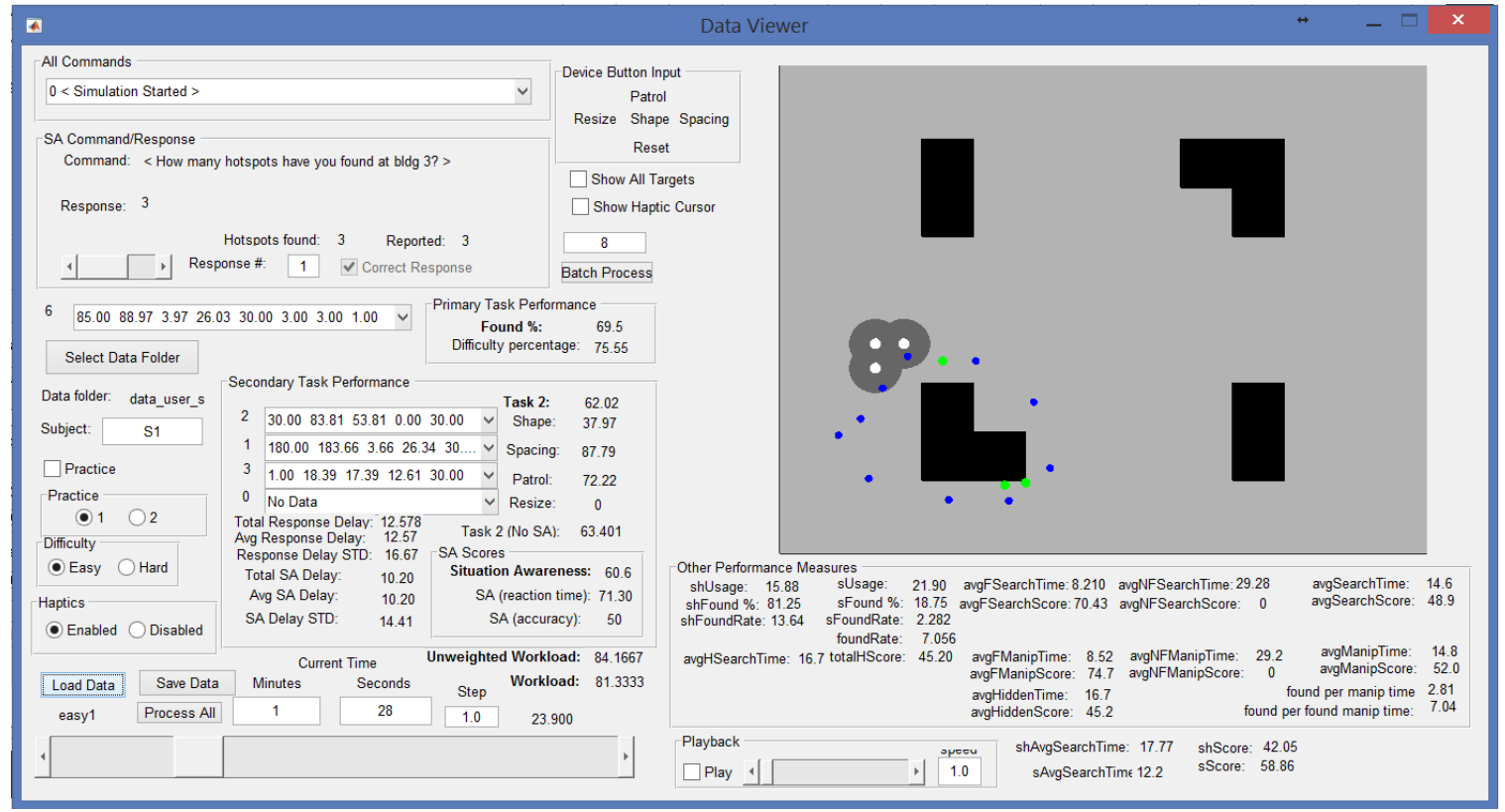

Figure 4.5: The Data Viewer displays each subject's team positions, haptic cursor position and button pressed throughout a session. This application also calculates the experimental measures to be analyzed.

\subsection{Chapter Summary}

This chapter has presented the purpose, experimental approach and design for the human subject experiment. Measures to evaluate operator performance in several areas were also explained. Results from processing experimental data from all participants and the statistical analysis are detailed in Chapter 5. 


\section{CHAPTER 5. RESULTS AND DISCUSSION}

This chapter details the statistical analysis and results from the human subject experiment. Each subject that participated in the experiment achieved a reasonable level of performance, so none of the data were omitted prior to the analysis. The analysis was performed in consultation with the BYU Center for Collaborative Research and Statistical Consulting. Once measures were obtained for each subject, the effects of various treatments of factors and levels were analyzed using a mixed models analysis of variance (ANOVA) with blocking on subject. A standard $95 \%$ confidence interval with $\alpha=0.05$ was used as the cutoff for statistical significance. The resulting $p$ values for main effects and interaction of factors are shown in Table 5.1.

Table 5.1: Effect $p$ values for all measures. Significant effects are in bold.

\begin{tabular}{|l|c|c|c|}
\hline Measure & Haptics & World & Haptics*World \\
\hline \hline Percent Hotspots Found & 0.0003 & $<0.0001$ & $\mathbf{0 . 0 3 9 2}$ \\
\hline Total Command Score & 0.3284 & 0.4436 & NA \\
\hline Total SA Score & 0.1521 & $\mathbf{0 . 0 0 4 2}$ & NA \\
\hline NASA TLX Workload & 0.6787 & $<0.0001$ & $\mathbf{0 . 0 3 5 2}$ \\
\hline Shape Time Percentage & $\mathbf{0 . 0 1 9 4}$ & 0.6201 & NA \\
\hline Percent Hotspots Found by Shape & 0.0675 & 0.101 & $\mathbf{0 . 0 4 7 4}$ \\
\hline Shape Hotspots Found Rate & $\mathbf{0 . 0 1 4 5}$ & $\mathbf{0 . 0 0 5 4}$ & NA \\
\hline Spacing Time Percentage & 0.2083 & 0.3951 & NA \\
\hline Hotspots Found by Spacing & 0.0675 & 0.101 & $\mathbf{0 . 0 4 7 4}$ \\
\hline Spacing Hotspots Found Rate & 0.2647 & 0.2037 & NA \\
\hline Hotspots Found Rate & 0.0011 & 0.018 & $\mathbf{0 . 0 0 2 4}$ \\
\hline Avg. Search Time & 0.9110 & $<\mathbf{0 . 0 0 0 1}$ & NA \\
\hline Avg. Manipulation Time & 0.1585 & $<\mathbf{0 . 0 0 0 1}$ & NA \\
\hline Avg. Team Command Response Time & 0.7085 & 0.926 & NA \\
\hline Avg. SA Response Time & 0.0706 & 0.5148 & NA \\
\hline SA Accuracy Score & 0.5388 & $\mathbf{0 . 0 0 2}$ & NA \\
\hline
\end{tabular}


As shown in the table, the analysis checked for significance of main effects from both factors as well as interactions between factors. If an interaction is significant, the analysis from the main effects can be misleading so it is ignored. In the following sections, the combinations of factors are listed as "NE" for no haptics (No) in an easy world, "YE" for haptics (Yes) in an easy world, "NH" for no haptics in a hard world, and "YH" for haptics in a hard world. This designation will be used to easily show a difference of means between treatments in the analysis. For example, the difference of means for a Haptics*World interaction would report NH-YH mean $=-12.3308$, which specifies that the no haptics in hard world mean minus the haptics in hard world mean is -12.3308. Likewise, the difference of means for a Haptics main effect would report $\mathrm{N}-\mathrm{Y}$ mean $=$ -2.4092 , which specifies that the no haptics mean minus the haptics mean is -2.4092 .

\subsection{Primary Measures}

\subsubsection{Percent Hotspots Found}

There was a significant interaction between haptic feedback and world difficulty $(\mathrm{p}=$ 0.0392). Table 5.2 summarizes the resulting significant interactions for this measure. There was no significant difference in interaction between haptic feedback and no haptic feedback in the easy world $(\mathrm{p}=0.2778)$, but haptic feedback did still improve performance overall (NE-YE mean $=$ -4.80549). This suggests that haptic feedback provides a relatively small advantage in finding hotspots when task demand is low.

Table 5.2: Significant differences

for percent hotspots found.

\begin{tabular}{|c|c|c|c|}
\hline Effect & Mean Diff & Std Error & P Value \\
\hline Haptics*World: NE-NH & 28.6107 & 2.4100 & $<0.0001$ \\
\hline Haptics*World: NE-YH & 16.2798 & 2.5942 & $<0.0001$ \\
\hline Haptics*World: YE-NH & 33.4161 & 2.5942 & $<0.0001$ \\
\hline Haptics*World: YE-YH & 21.0853 & 2.4100 & $<0.0001$ \\
\hline Haptics*World: NH-YH & -12.3308 & 2.5785 & 0.0008 \\
\hline
\end{tabular}

All four differences that compare levels of easy world difficulty to hard world difficulty were significant, which simply shows that the range of difficulty between the two levels was rea- 
sonable for the experiment. In the hard world, there were more hotspots to locate and they expired more rapidly, which created a more urgent need for the operator to switch view between both displays.

When comparing haptic feedback with no haptic feedback in the hard world, the presence of haptic feedback significantly improved the operator's ability to find hotspots, as shown in Figure 5.1. One reason for this could be that the operator was more capable at managing the team with shape or spacing manipulations when haptic feedback was enabled. The operator could have used the haptic feedback to more quickly orient the haptic cursor relative to the team in preparation for the next manipulation. Since haptic feedback allows the operator to feel the shape instead of just see it, the operator could look at the sensor readings on the secondary display without even looking at the primary display for periods of time. Observations of the subjects' performance throughout the experiment show that some or all of these conclusions are reasonable. Results from the postexperiment questionnaire showed that on average, subjects preferred the use of haptic feedback overall (rating of 3.7 on a scale from 1 to 5), which is consistent with statistical results. It is clear that haptic feedback did improve performance, and since haptic feedback provides subjects with team information without binding them to a visual display, a more heads-up approach can be encouraged. This improved performance shows that heads-up control of the team can be more easily achieved with haptic feedback than without it.

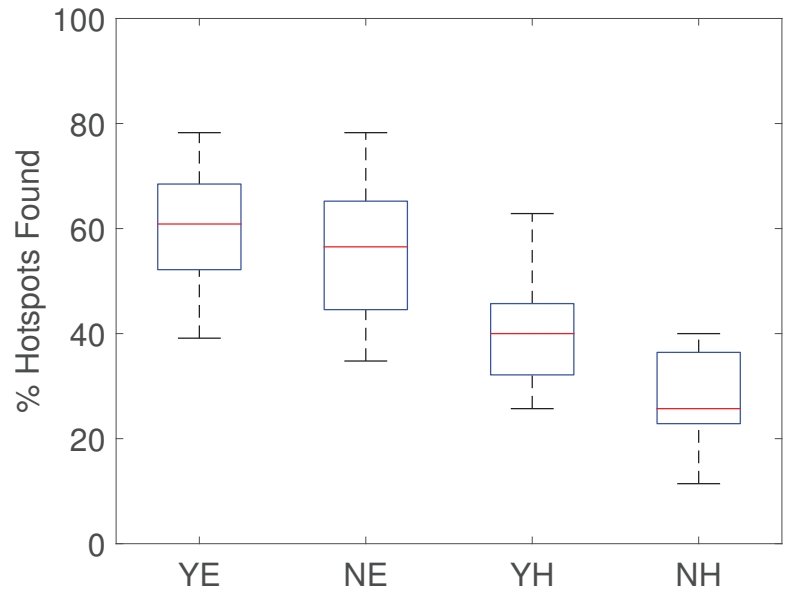

(a)

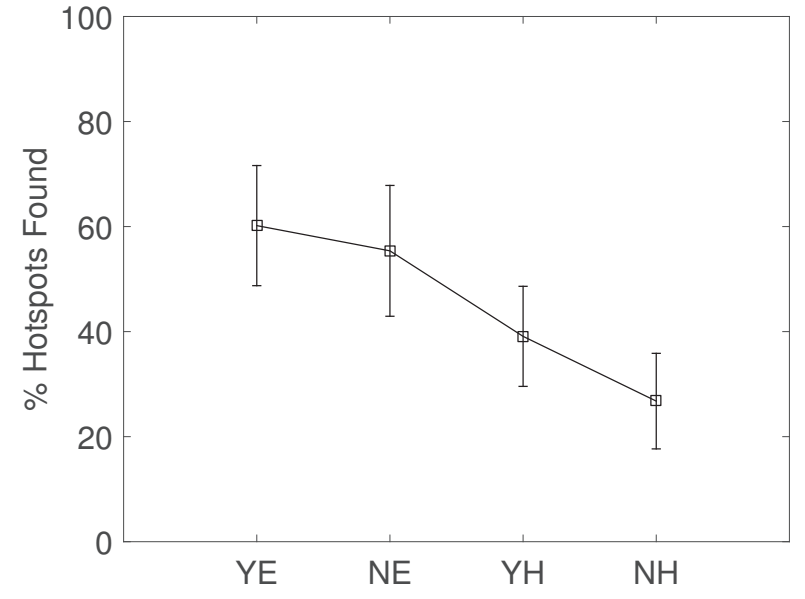

(b)

Figure 5.1: Boxplots (a) and mean/standard deviation plots (b) for percent hotspots found 


\subsubsection{Total Command Score}

There were no significant effects due to haptic feedback or world difficulty on the total command score measure. Since responding to incoming commands relating to team manipulation or answering SA questions was a secondary task, haptic feedback was not expected to make as much of a difference on this measure. It was, however, hoped that there would be some significant difference overall when subjects were provided haptic feedback. Upon inspection of Figure 5.2, there was a slight trend suggesting that there was an advantage to using haptic feedback (N-Y mean $=-1.4176$ ), but that is likely due to the SA response time measure (see Section 5.2.10), which partially contributes to this collective score.

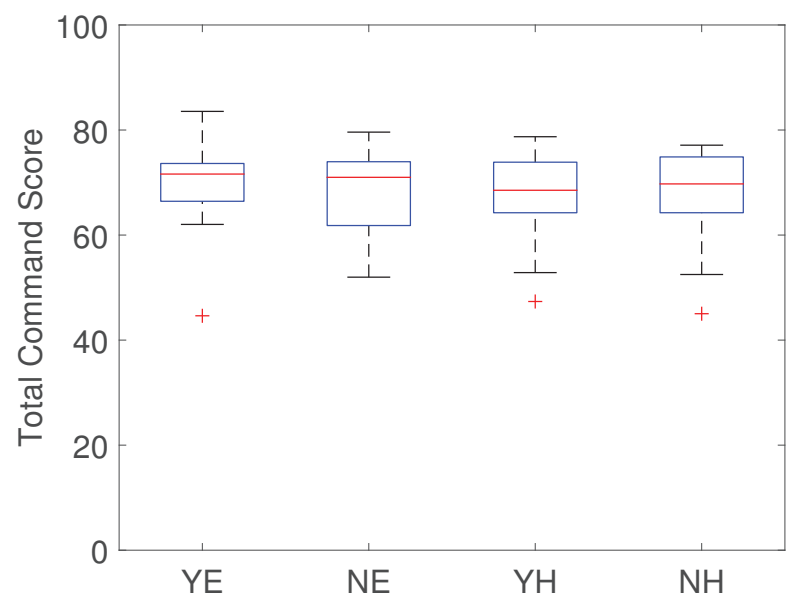

(a)

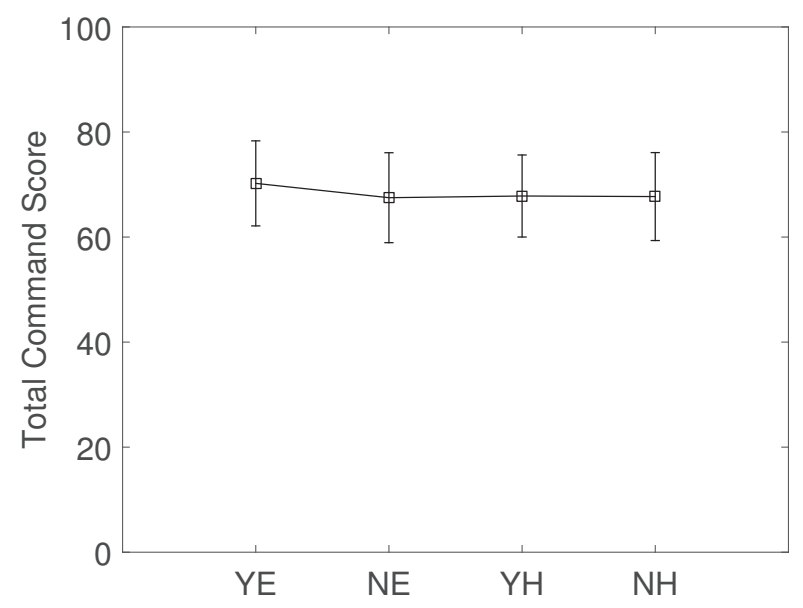

(b)

Figure 5.2: Boxplots (a) and mean/standard deviation plots (b) for total command score

The effectiveness of haptic feedback during travel mode was also evaluated by only considering the operator's response to travel commands. Results for that sub-measure resulting in insignificant differences due to haptic feedback. On average, the subject preference rating of haptic feedback during travel mode was 3.1 on a scale from 1 to 5 . Since traveling to another building was a relatively straightforward objective in travel mode, it is not surprising that haptic feedback did not play a large role in an operator's travel performance. In this work, the travel mode was necessary in order to move the team from building to building, but the experiment was not designed to measure the effectiveness of the feedback in this mode as heavily as shape or spacing manipulations in surround mode. 
As for the effect due to world difficulty, it is minimal in this measure because all team commands and SA questions were designed to be easier in the easy world or more difficult in the hard world. The lack of significant difference is therefore expected and preferred in order to more easily interpret other measures.

\subsubsection{Total SA Score}

A significant difference was found when only considering the effect of world difficulty on SA score $(p=0.0042)$. The questions asked to the operator to determine this score were very similar between worlds, so that is likely not to be the cause of this difference. The easy world resulted in a higher SA score than that of the hard world (see Table 5.3).

Table 5.3: Significant differences for total SA score.

\begin{tabular}{|c|c|c|c|}
\hline Effect & Mean Diff & Std Error & P Value \\
\hline World: E-H & 5.4759 & 1.6726 & 0.0042 \\
\hline
\end{tabular}

This result suggests that overall, subjects tended to have a higher level of SA while managing the team in an easy environment than in a hard environment (See Figure 5.3). If it were

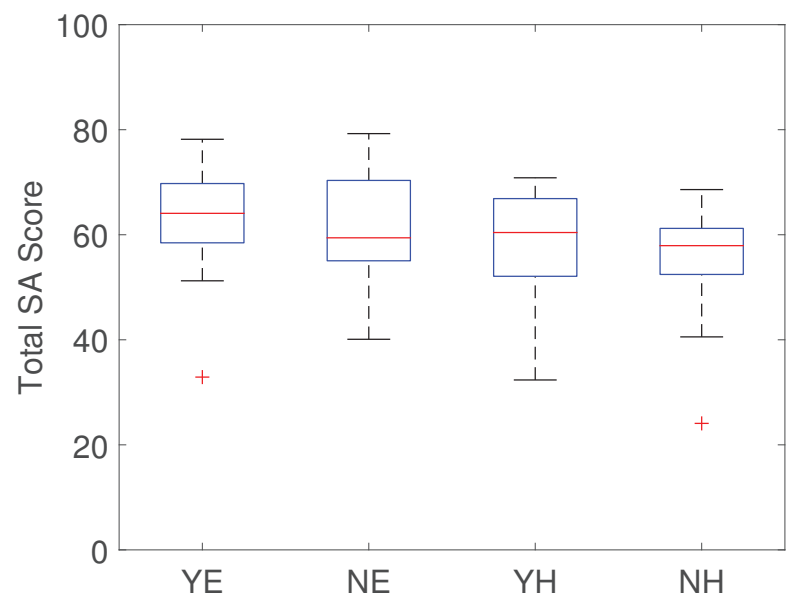

(a)

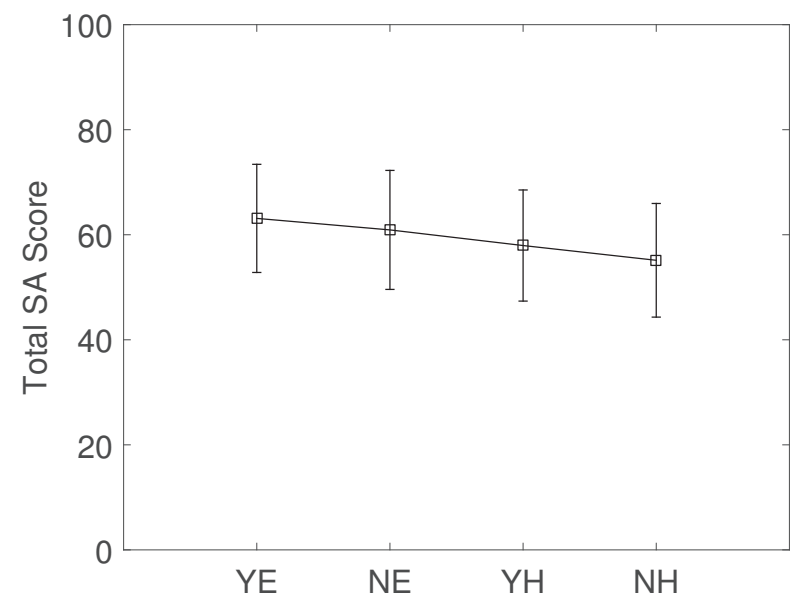

(b)

Figure 5.3: Boxplots (a) and mean/standard deviation plots (b) for total SA score 
not so, less confidence would need to be placed on the development of the 3-Q model-based SA evaluation. Therefore, there is reason to suppose that this method of objective SA measurement is valid.

Haptic feedback did not significantly effect SA score. There was, however, an increase in SA Score with haptic feedback $(\mathrm{N}-\mathrm{Y}$ mean $=-2.5017)$, which can also be noticeably seen in Figure 5.3b. Marginal significance was found in the SA response time measure (see Section 5.2.10) due to haptic feedback, which may be the likely cause of this trend. These results suggest that haptic feedback did not significantly improve overall SA. The upward trend does support the idea that it does not degrade SA, which is also important.

\subsubsection{NASA TLX Workload}

The NASA TLX workload measure returned with significant interaction between haptic feedback and world difficulty $(\mathrm{p}=0.0352)$. Only one significant difference was found between haptics in the easy world and haptics in the hard world, as shown in Table 5.4. This suggests that workload increases when managing the team in a harder world, which is to be expected due to a higher task demand. When task demand is kept low, as is the case in an easy world, the operator workload is lower and the presence of haptic feedback has little effect.

Table 5.4: Significant differences

for NASA TLX workload.

\begin{tabular}{|c|c|c|c|}
\hline Effect & Mean Diff & Std Error & P Value \\
\hline Haptics*World: YE-YH & -11.0877 & 2.1001 & 0.0003 \\
\hline
\end{tabular}

The NASA TLX is a subjective measure of workload, and each subject has a different standard of "low" or "high" workload when ratings are submitted throughout the experiment. This makes it challenging to objectively determine the actual workload experienced by the operator.

From viewing the results in Figure 5.4, there is a slight increase in workload when comparing haptics to no haptics in the hard world $(\mathrm{NH}-\mathrm{YH}$ mean $=-4.7579)$. This difference, however, is not statistically significant and may even be caused by inconsistency of ratings between sessions or a misinterpretation of subscale definitions. Therefore, from the results of this experiment, haptic 


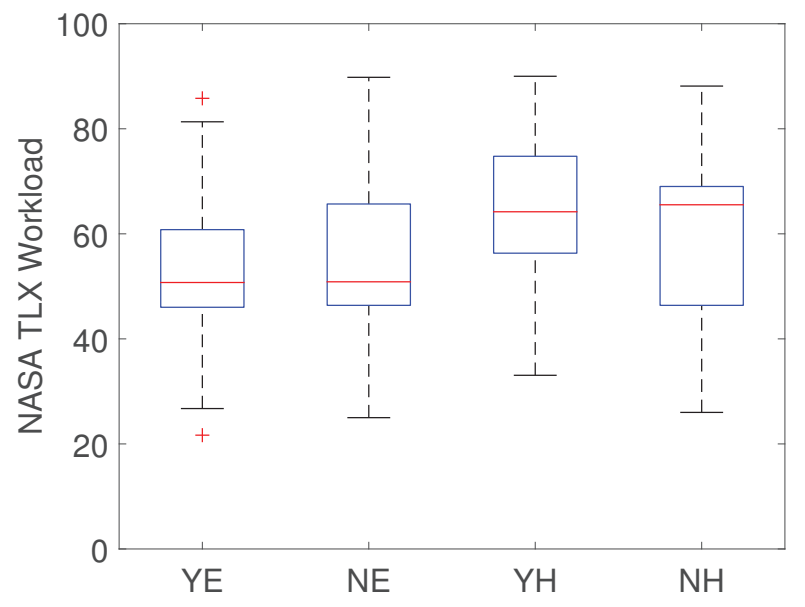

(a)

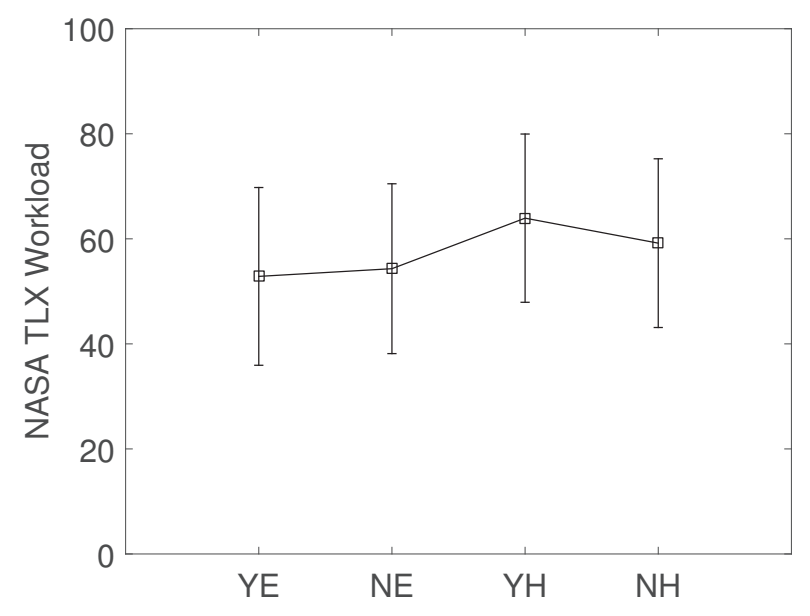

(b)

Figure 5.4: Boxplots (a) and mean/standard deviation plots (b) for NASA TLX workload

feedback alone had no significant effect on the operator's level of workload while managing the team.

\subsection{Secondary Measures}

\subsubsection{Shape Time Percentage}

In general, haptic feedback significantly increased the percentage of time that the SHAPE button was engaged (see Table 5.5). Part of the strategy to finding hotspots involved viewing the secondary display with sensor readings while manipulating the shape of the team at the same time. An increased shape time percentage with haptics feedback suggests that the operator spent more

Table 5.5: Significant differences

for shape time percentage.

\begin{tabular}{|c|c|c|c|}
\hline Effect & Mean Diff & Std Error & P Value \\
\hline Haptics: N-Y & -2.4092 & 0.9386 & 0.0194 \\
\hline
\end{tabular}

time engaging the SHAPE button and attempting to move the team as opposed to being disengaged and orienting the haptic cursor with respect to the team. Since changing the shape of the team was a primary method to search different regions around the building, more engagement of the SHAPE 
button suggests an increased ability or tendency to do so. Differences of this measure due to world difficulty were not statistically significant, as shown in Figure 5.5. The slight increase due to haptic feedback is also noticeable.

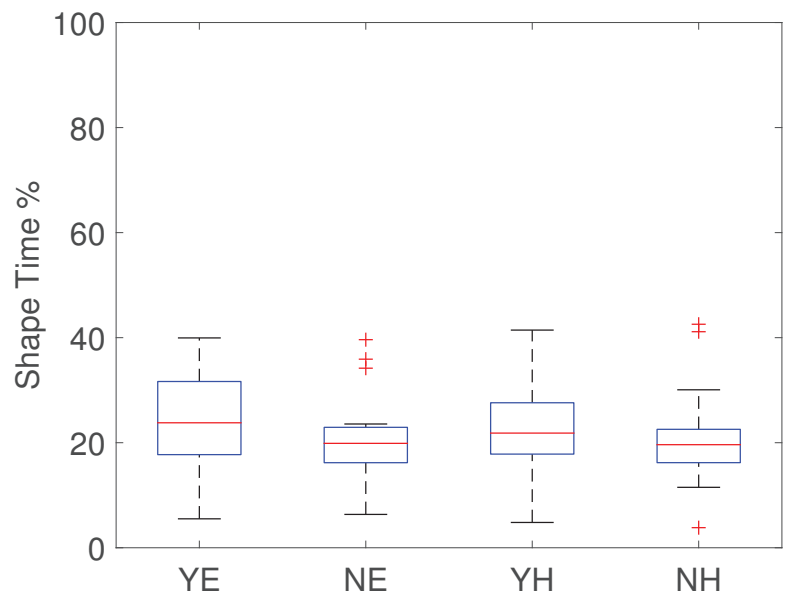

(a)

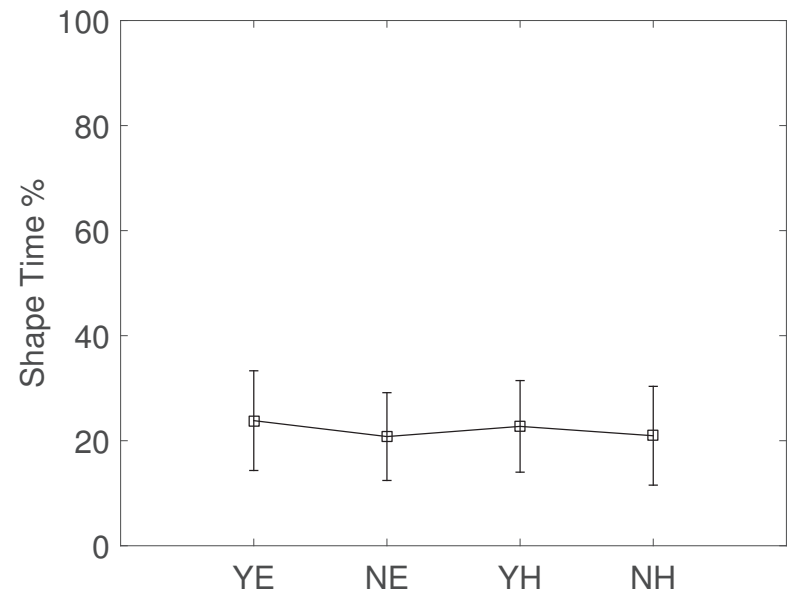

(b)

Figure 5.5: Boxplots (a) and mean/standard deviation plots (b) for shape time percentage

\subsubsection{Percent Hotspots Found by Shape}

The percent of hotspots found by using shape manipulation was significantly influenced by the interaction between haptic feedback and world difficulty $(\mathrm{p}=0.0474)$. Two of the significant differences in this interaction were between no haptics in the hard world, and both haptics and no haptics in the easy world, as shown in Table 5.6. Similar to the percent hotspots found measure,

Table 5.6: Significant differences for percent hotspots found by shape.

\begin{tabular}{|c|c|c|c|}
\hline Effect & Mean Diff & Std Error & P Value \\
\hline Haptics*World: NE-NH & 18.2805 & 5.1125 & 0.0106 \\
\hline Haptics*World: YE-NH & 19.2193 & 5.5786 & 0.0140 \\
\hline Haptics*World: NH-YH & -13.3557 & 4.6915 & 0.0480 \\
\hline
\end{tabular}

these differences simply validate that the hard world was indeed more difficult than the easy world when it came to finding hotspots. The other significant difference between no haptics and haptics 
in the hard world shows that haptic feedback improves the operator's ability to find hotspots using shape manipulation. There was not a significant difference when comparing levels of haptic feedback in the easy world, although haptic feedback did slightly improve the percentage (NE-YE mean $=-0.9388$ ), as shown in Figure 5.6. The average preference rating for use of haptic feedback during shape manipulation was also high, rating a 3.6 on a scale from 1 to 5 , which is very close to the overall haptic feedback preference rating of 3.7 .

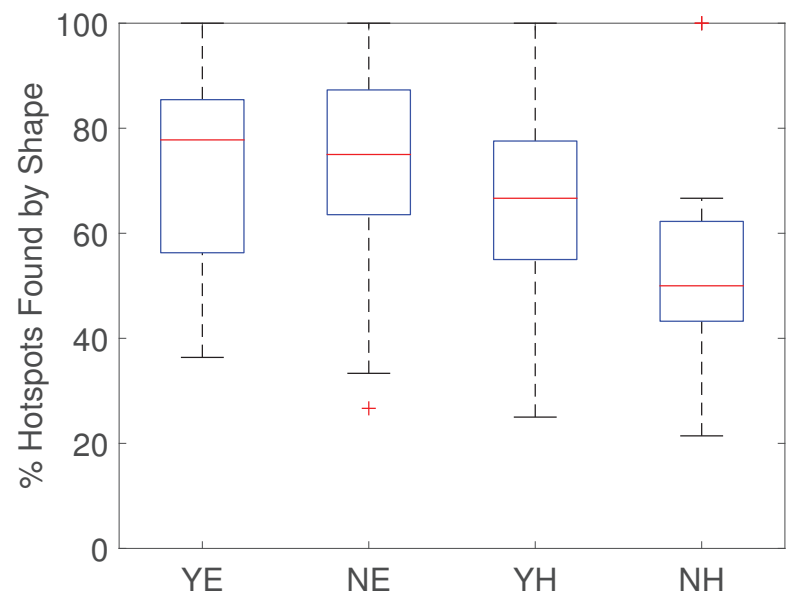

(a)

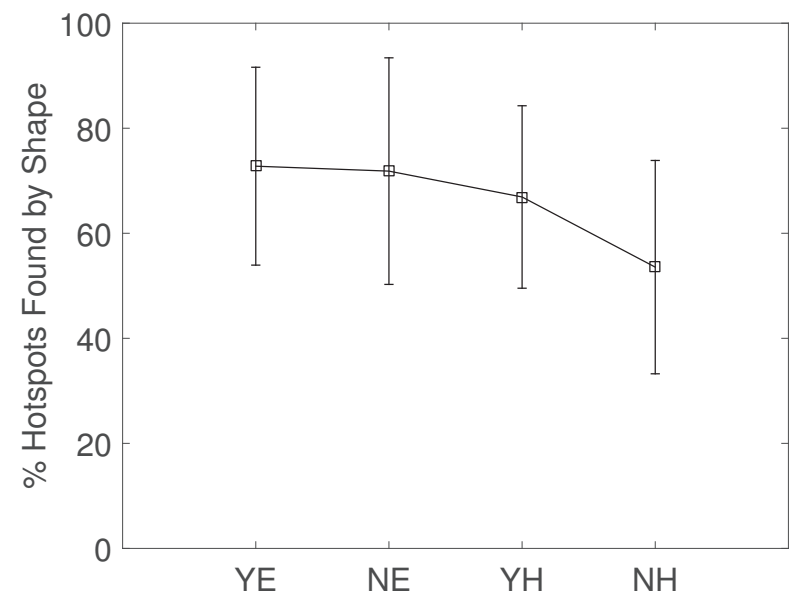

(b)

Figure 5.6: Boxplots (a) and mean/standard deviation plots (b) for percent hotspots found by shape

These results are nearly identical to those acquired with the percent hotspots found measure, which shows that an operator's ability to use shape manipulation to find hotspots is a good predictor for success in finding hotspots as a whole. This is especially evident when observing the trend in percent hotspots found by spacing manipulation, which is the only other way to find hotspots and has the opposite trend with the same significant differences. For example, the percentage of hotspots found by shape manipulation with haptics in the easy world is $71.8 \%$, so the percentage of hotspots found by spacing manipulation would be $28.2 \%$. As shown in Figure 5.6, the average percentage of hotspots found by shape manipulation is always higher than that of spacing manipulation in all levels of haptic feedback and world difficulty.

In comparing this trend to percent hotspots found in Section 5.1.1, one can conclude that the operator finds more hotspots by shape than spacing manipulation. The average preference rating for the use of haptic feedback during spacing manipulation was 3.7 on a scale from 1 to 5 . 
Since the preference is high, using haptic feedback during spacing manipulation may still improve the mode of control, but not have a great influence on operator performance as defined by the experiment.

Furthermore, since haptic feedback provided an advantage in finding hotspots with shape manipulation, which required the operator to view both screens for team and sensor information, this also supports the notion that haptic feedback improves the operator's ability to maintain headsup control of the team.

\subsubsection{Shape Hotspots Found Rate}

In general, haptic feedback did improve the operator's efficiency in finding hotspots using shape manipulation (see Table 5.7).

Table 5.7: Significant differences

for shape hotspots found rate.

\begin{tabular}{|c|c|c|c|}
\hline Effect & Mean Diff & Std Error & P Value \\
\hline World: E-H & 1.9358 & 0.6130 & 0.0054 \\
\hline Haptics: N-Y & -1.6582 & 0.6130 & 0.0145 \\
\hline
\end{tabular}

As shown in Figure 5.7, the rate increases when haptic feedback is present, especially in the hard world. These results also suggest that the operator's ability to use shape manipulation to find hotspots is improved with haptic feedback when the task demand is higher. There was also a significant difference between easy and hard worlds $(p=0.0054)$ for this measure. Since shape time percentage showed no significant differences due to world difficulty (see Section 5.2.1), the rate at which hotspots are found using shape manipulation follows a similar trend of the percent hotspots found by shape, explained in the previous section. The results for this efficiency measure show that haptic feedback not only helps the operator find more hotspots, but it helps the operator do so in fewer button presses or manipulations. This is important because in the experiment and in real scenarios, there is a limited amount of time to perform reconnaissance. Many of the hotspots that would have remained hidden upon expiration were found and counted toward the operator's overall performance. 


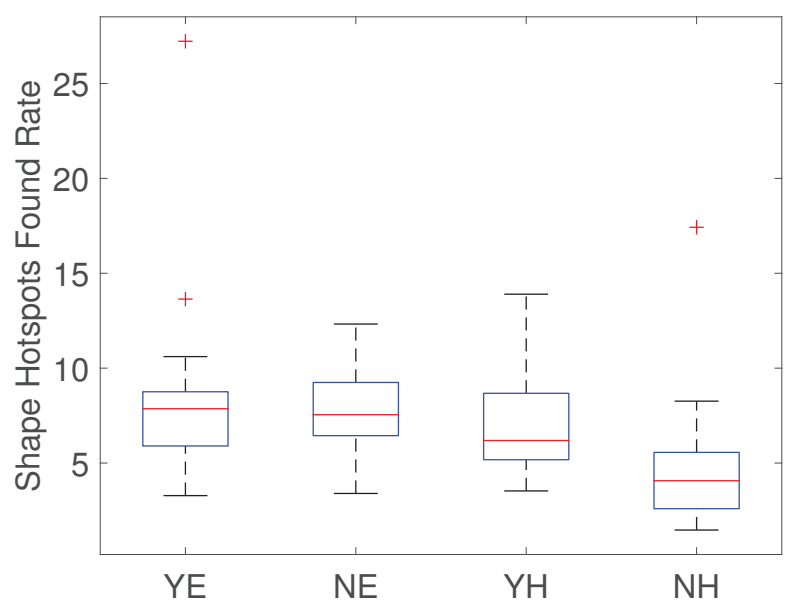

(a)

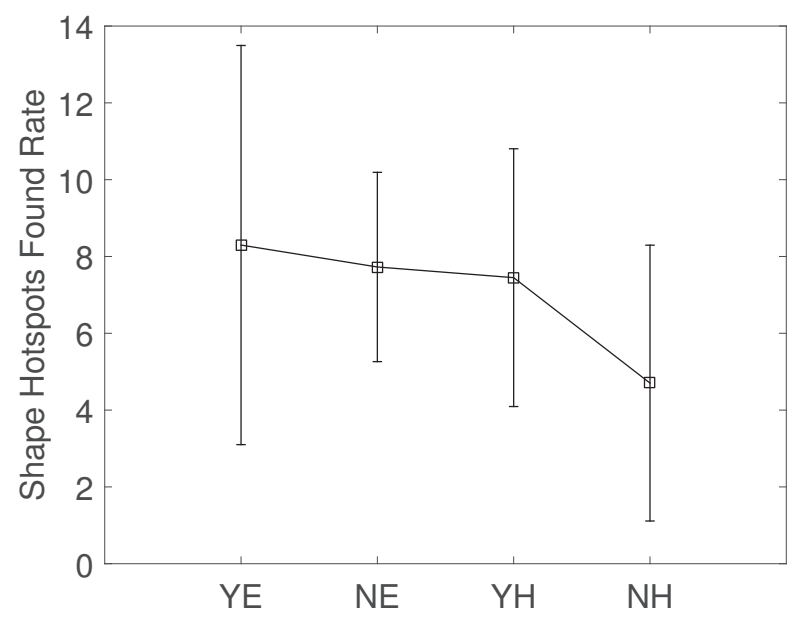

(b)

Figure 5.7: Boxplots (a) and mean/standard deviation plots (b) for shape hotspots found rate

\subsubsection{Spacing Time Percentage}

There were no significant effects due to haptic feedback or world difficulty levels on the percentage of time that the operator managed the team by spacing manipulation. As can be seen in Figure 5.8, there is not much change in this measure across all levels of haptic feedback or world difficulty. When comparing these results to those of shape time percentage, it appears that spacing manipulation is used just as often, although there seems to be no benefit to using haptic feedback.

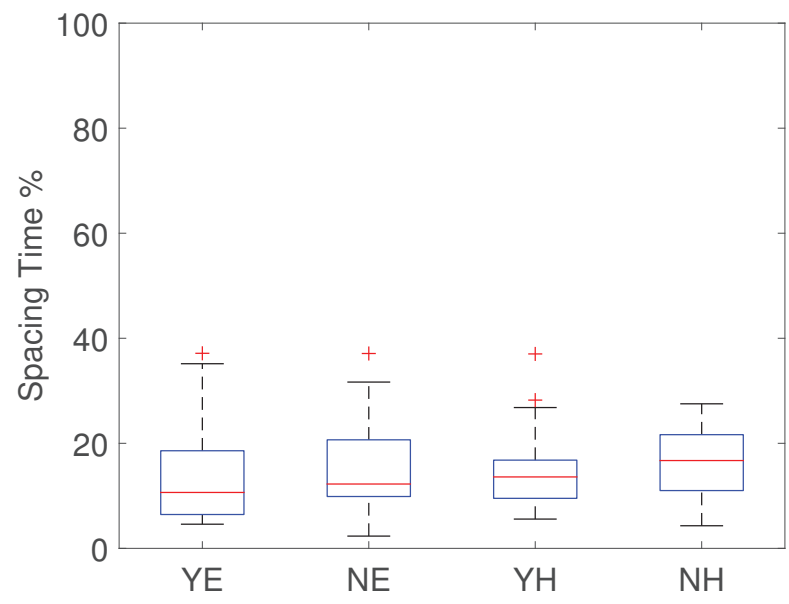

(a)

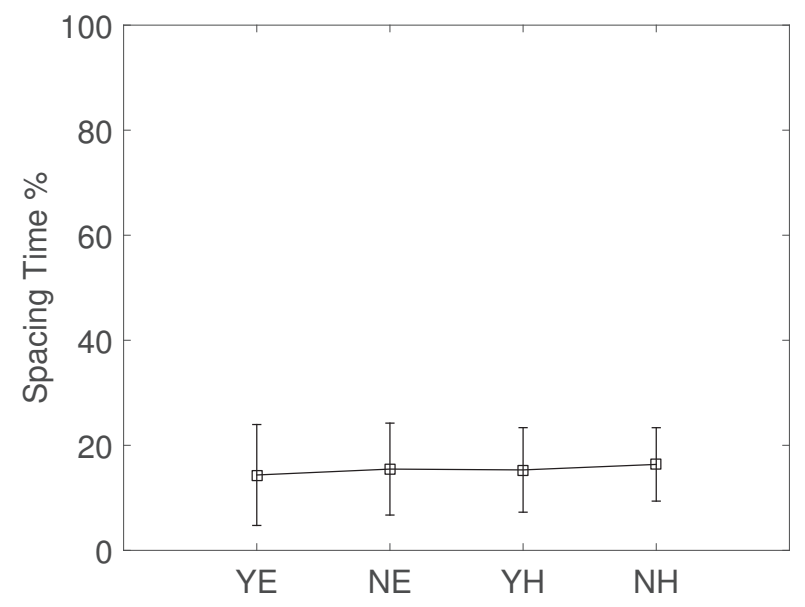

(b)

Figure 5.8: Boxplots (a) and mean/standard deviation plots (b) for spacing time percentage 
This result is reasonable because the mode of interaction for spacing manipulation does not require the operator to view the main team display as much as shape manipulation. The operator may quickly place more spacing from one side of the formation to the other without even looking at the team display. Therefore, haptic feedback was not shown to be as advantageous for spacing manipulation as it was for shape manipulation. Compared to shape time percentage in Section 5.2.1, the amount of time used on spacing manipulation (see Figure 5.8) was slightly less. This is likely due to the operator recognizing that more hotspots can be searched for and found by shape rather than spacing manipulation.

\subsubsection{Spacing Hotspots Found Rate}

No significant effects due to haptic feedback or world difficulty were present in this measure. By inspecting Figure 5.9b, haptic feedback does seem to cause a slight improvement (N-Y mean $=-0.6647)$, but the amount of variation in the data causes a lack of significance in this trend. These results suggest that haptic feedback did not significantly improve or hinder the operator's efficiency in using spacing manipulation to find hotspots. Once again, this is a reasonable result because, unlike shape manipulation, there is less of a need to identify the precise location of the haptic cursor relative to the team, thus making the effectiveness of haptic feedback a matter of preference.

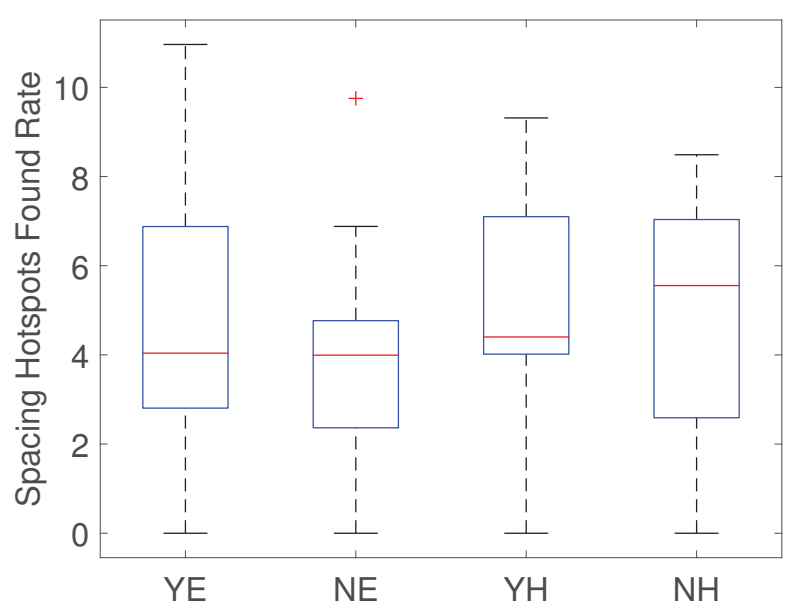

(a)

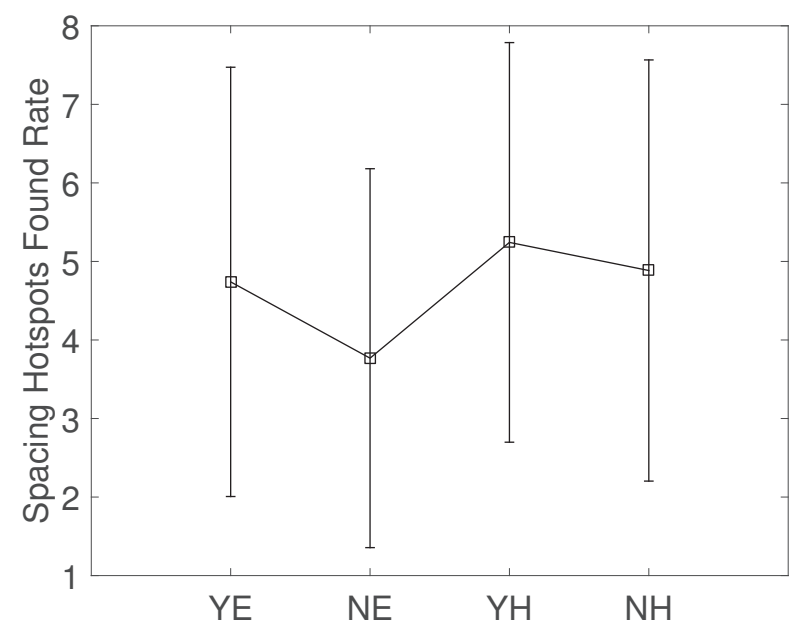

(b)

Figure 5.9: Boxplots (a) and mean/standard deviation plots (b) for spacing hotspots found rate 


\subsubsection{Hotspots Found Rate}

The hotspots found rate measure did result in significant interaction between haptic feedback and world difficulty $(\mathrm{p}=0.0024)$. Results for this measure are very similar to the percent hotspots found measure presented in Section 5.1.1. The presence of haptic feedback significantly improves the operator's time efficiency in finding hotspots in the hard world (see Table 5.8). When

Table 5.8: Significant differences

for hotspots found rate.

\begin{tabular}{|c|c|c|c|}
\hline Effect & Mean Diff & Std Error & P Value \\
\hline Haptics*World: NE-NH & 1.7743 & 0.3936 & 0.0014 \\
\hline Haptics*World: YE-NH & 1.8723 & 0.3936 & 0.0008 \\
\hline Haptics*World: NH-YH & -2.0632 & 0.3936 & 0.0003 \\
\hline
\end{tabular}

comparing Figure 5.10 to the shape hotspots found rate results in Figure 5.7, one can see that the operator's overall time efficiency in finding hotspots is largely determined by how quickly the operator finds hotspots using shape manipulation. In both cases, haptic feedback helped to improve efficiency and allow the operator to perform better in the primary task of finding hotspots.

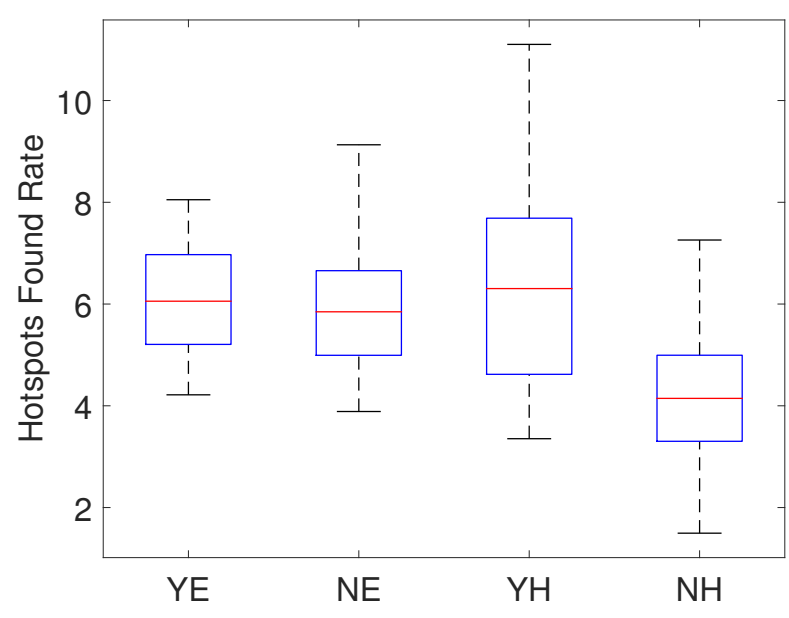

(a)

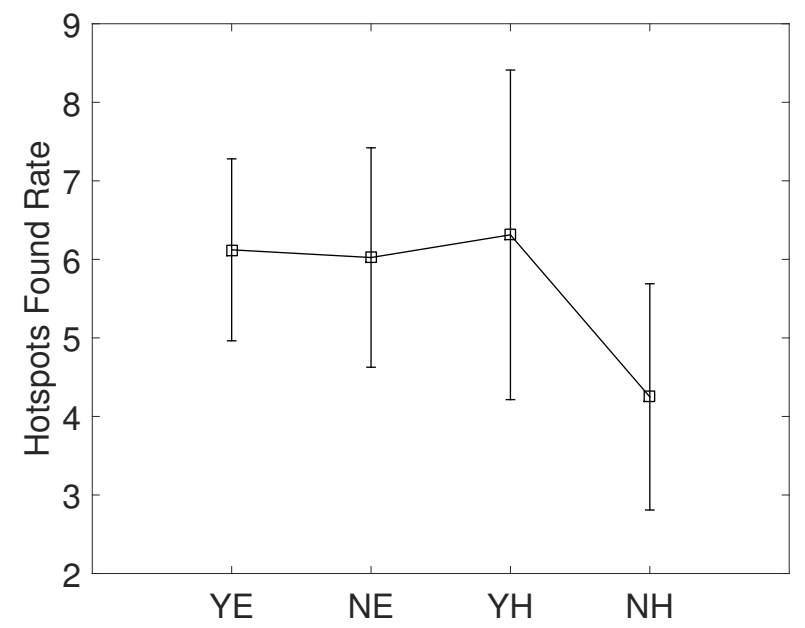

(b)

Figure 5.10: Boxplots (a) and mean/standard deviation plots (b) for hotspots found rate 


\subsubsection{Average Search Time}

The average search time returned with significant effects due to world difficulty (see Table 5.9), but not due to haptic feedback. Due to search time being defined as the time in between when a hotspot first becomes active and when it is in range of the team, minimizing this time is not always in the operator's control. There is an inevitable "luck factor" involved in finding some hotspots. The operator may be searching very close to or in an entirely different area than where hotspots are appearing. Therefore, haptic feedback did not have a large effect on this measure.

Table 5.9: Significant differences

for search time.

\begin{tabular}{|c|c|c|c|}
\hline Effect & Mean Diff & Std Error & P Value \\
\hline World: E-H & 5.1455 & 0.5263 & $<0.0001$ \\
\hline
\end{tabular}

The significant difference between easy and hard worlds, as shown in Figure 5.11, can be attributed to an increased number of hotspots in the world while keeping the total simulation time the same. This caused hotspots to appear more rapidly and also encouraged the subjects to act more quickly in moving the team to various regions around the building. Some hotspots in the hard world were actually easier to initially detect, but more difficult to uncover due to there being multiple hotspots in the group. For these reasons, there were shorter search times in the hard world.

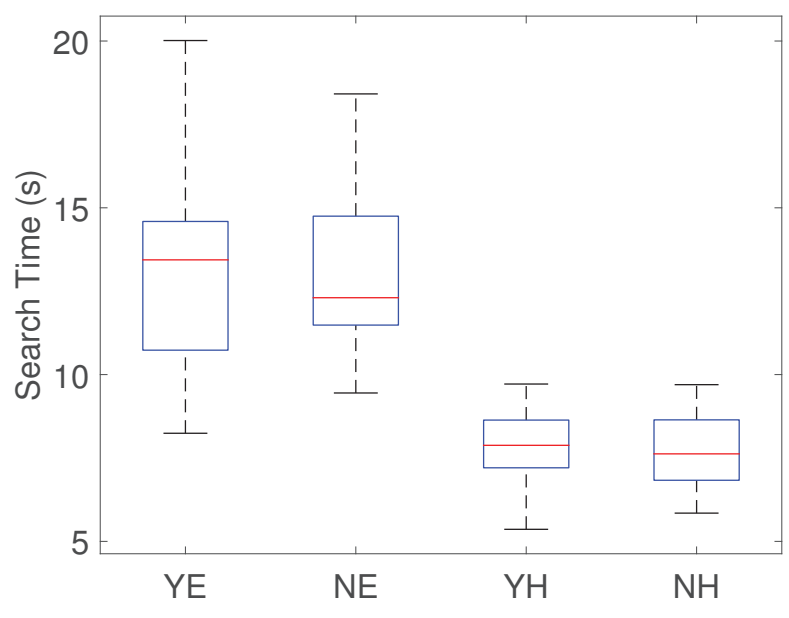

(a)

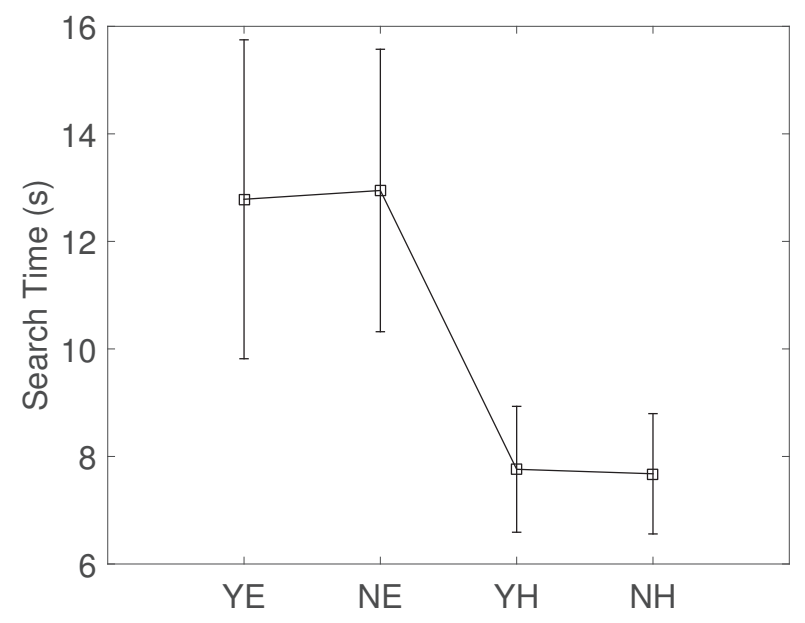

(b)

Figure 5.11: Boxplots (a) and mean/standard deviation plots (b) for search time 


\subsubsection{Average Manipulation Time}

There was a significant difference in average manipulation time due to world difficulty, but not due to haptic feedback, as shown in Table 5.10. The greater manipulation time in the easy world is partly due to longer active duration lengths of hotspot groups.

\section{Table 5.10: Significant differences for manipulation time.}

\begin{tabular}{|c|c|c|c|}
\hline Effect & Mean Diff & Std Error & P Value \\
\hline World: E-H & 2.0695 & 0.4049 & $<0.0001$ \\
\hline
\end{tabular}

Although not significant, there was a noticeable decrease in manipulation time with haptic feedback $(\mathrm{N}-\mathrm{Y}$ mean $=0.5612)$, as shown in Figure 5.12. Low values for manipulation time suggest that the operator was able to find hotspots faster once they come in range of the team, either by manipulating shape or spacing of the team. This shows, at least, that haptic feedback does not hinder the operator's ability to do this, but there is not enough evidence to suggest that haptic feedback does significantly improve manipulation time. Regardless of how quickly the operator was able to find hotspots, it is still just as important that they were eventually found before expiring. Haptic feedback does improve that outcome, as explained previously.

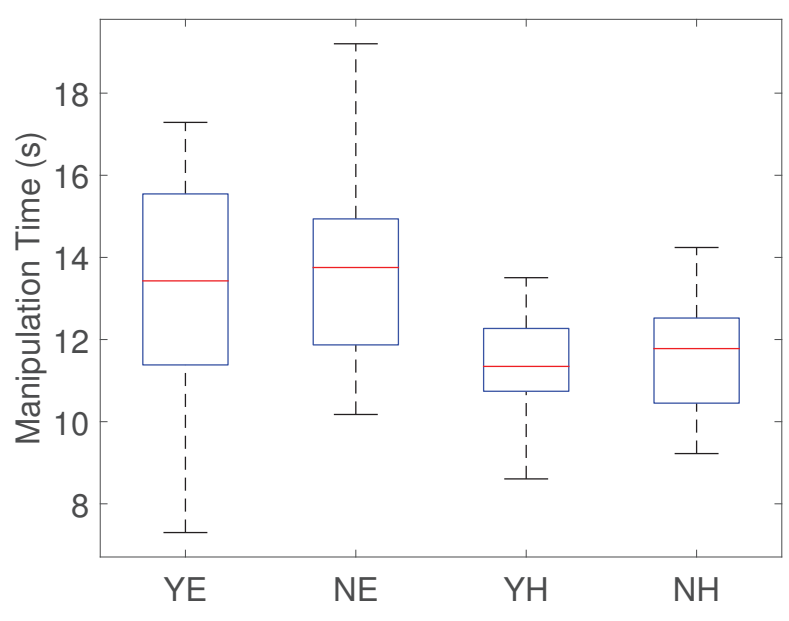

(a)

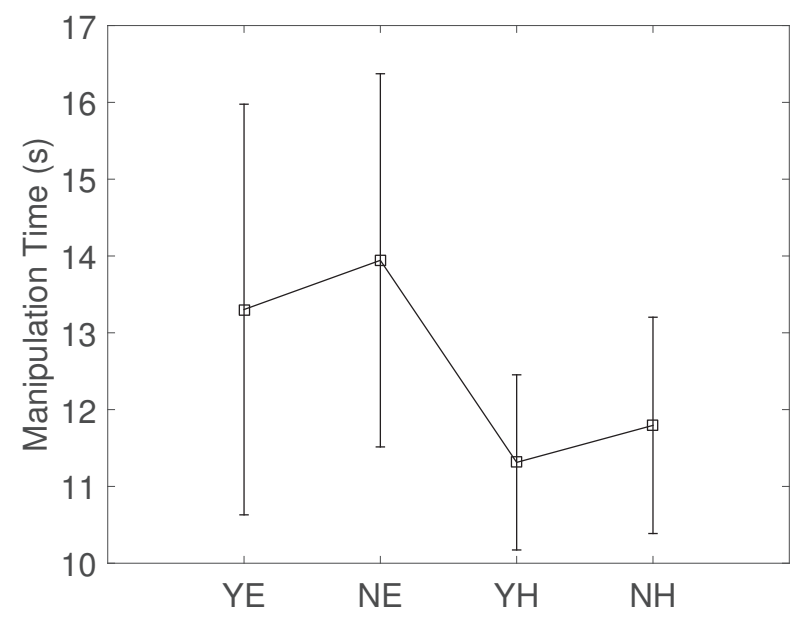

(b)

Figure 5.12: Boxplots (a) and mean/standard deviation plots (b) for manipulation time 


\subsubsection{Average Team Command Response Time}

There were no significant effects due to haptic feedback or world difficulty on the operator's response time to team manipulation commands, as shown in Figure 5.13. By observing how each subject responded to these commands throughout the experiment, most of the delay was caused by ill-attention to the incoming commands. They were not designed to be more difficult in the hard world, and both worlds had the same number of team related commands. Thus, having no effects due to world difficulty is justified.

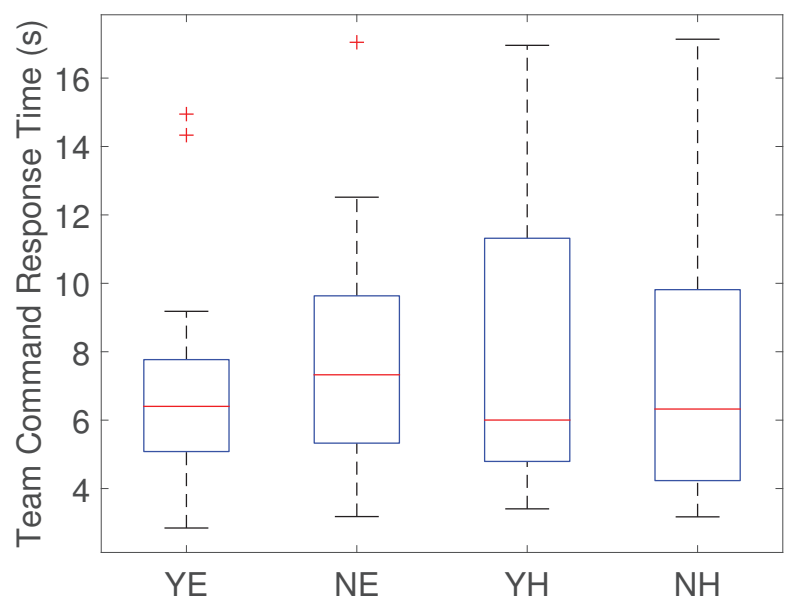

(a)

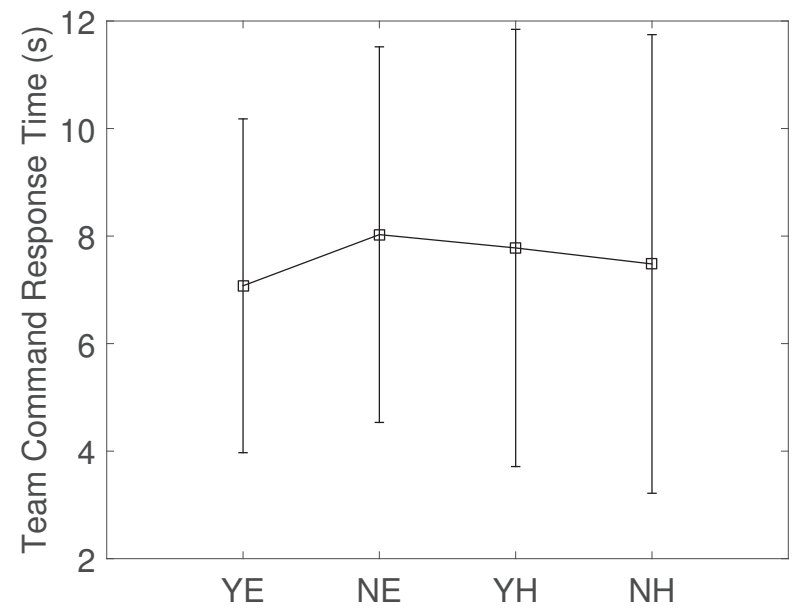

(b)

Figure 5.13: Boxplots (a) and mean/standard deviation plots (b) for team command response time

Given that a large portion of the response time was simply due to recognizing when a new command has been issued, it is not surprising that haptic feedback did not significantly help the operator in responding quickly. At best, haptic feedback would provide an increased awareness of the team shape or spacing to help the operator move into position more quickly and shorten the response time. Overall, haptic feedback did decrease response time by a small amount (N-Y mean $=0.3269)$. These results show that any decrease in response time due to haptic feedback is too small to give the operator a significant advantage. This begs to question whether developing additional haptic feedback specifically designed to help the operator respond to incoming messages might be beneficial. This must be approached with caution, because too many feedback modes could confuse the operator just as much as visual overload, which is to be avoided. 


\subsubsection{Average SA Response Time}

There were no significant differences due to haptic feedback or world difficulty in measuring an operators' average response time to SA questions. On average, the subjects took less time to respond in easy worlds than in hard worlds $(\mathrm{E}-\mathrm{H}$ mean $=-0.7296)$. This helps to validate the SA measurement method because one would expect a decrease in SA when multitasking demand is more intensive. Once again, this allows more confidence to be placed on the method, but does not guarantee its ability to accurately measure SA.

Haptic feedback did improve SA response time $(\mathrm{N}-\mathrm{Y}$ mean $=1.6715)$ overall, and was found to be marginally significant $(\mathrm{p}=0.0706)$. As shown in Figure 5.14b, there is a noticeable trend favoring haptic feedback in both easy and hard worlds, and variation in response times is also slightly smaller. This marginally significant result suggests that haptic feedback helps the operator have an increased awareness while performing the tasks, enough to respond faster to SA questions. Since haptic feedback helps the operator find hotspots, and SA questions are intended to test the operator's knowledge of hotspots around the environment, haptic feedback may allow the operator to focus on managing the team at a higher level. This attention on the team may enable the operator to recall or process task information more quickly, thus becoming more aware of the situation.

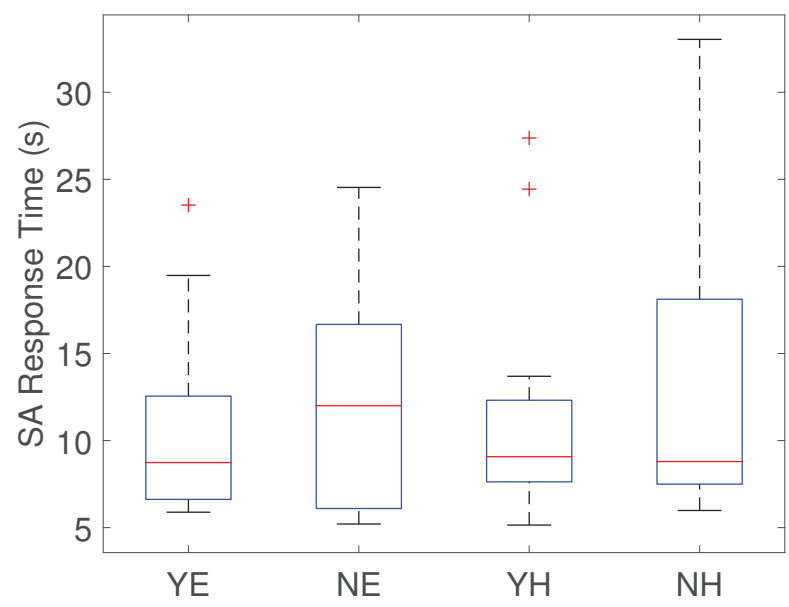

(a)

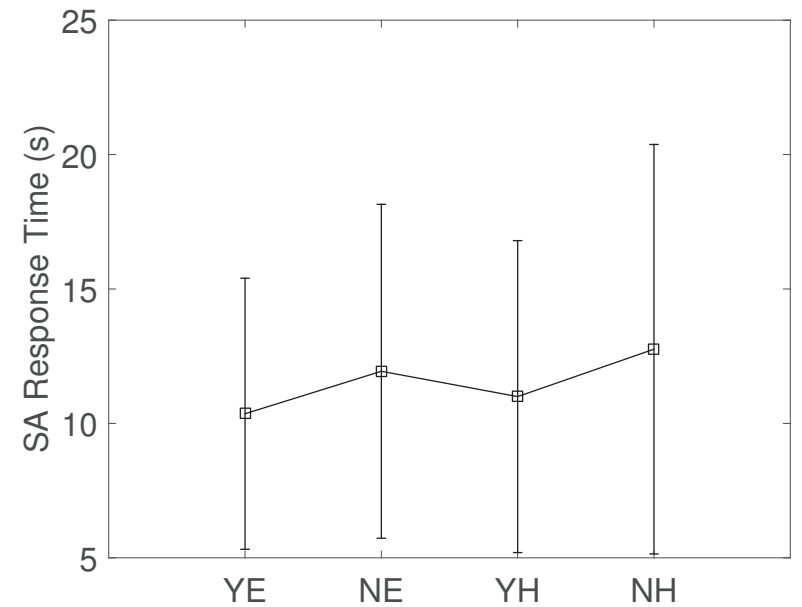

(b)

Figure 5.14: Boxplots (a) and mean/standard deviation plots (b) for SA response time 


\subsubsection{SA Accuracy Score}

There was a significant difference to SA accuracy score due to world difficulty (see Table 5.11), but not due to haptic feedback. When having the operator give responses to questions of equal difficulty between easy and hard worlds, there was a significantly higher score in the easy world than compared to the hard world.

Table 5.11: Significant differences

for SA accuracy score.

\begin{tabular}{|c|c|c|c|}
\hline Effect & Mean Diff & Std Error & P Value \\
\hline World: E-H & 10.0473 & 2.7861 & 0.0020 \\
\hline
\end{tabular}

This suggests that the operator had greater task awareness in the easy world because those responses were more accurate. As explained in Section 4.3.4, the SA accuracy score is equally weighted with the SA response time score to produce the total SA score. Based on these results, the SA accuracy score is useful in measuring key aspects of SA that are not covered by the SA response time measure. One would expect the operator's SA to decrease in an environment with higher task demand, which is represented by this measure. Scores from each subject are shown in Figure 5.15.

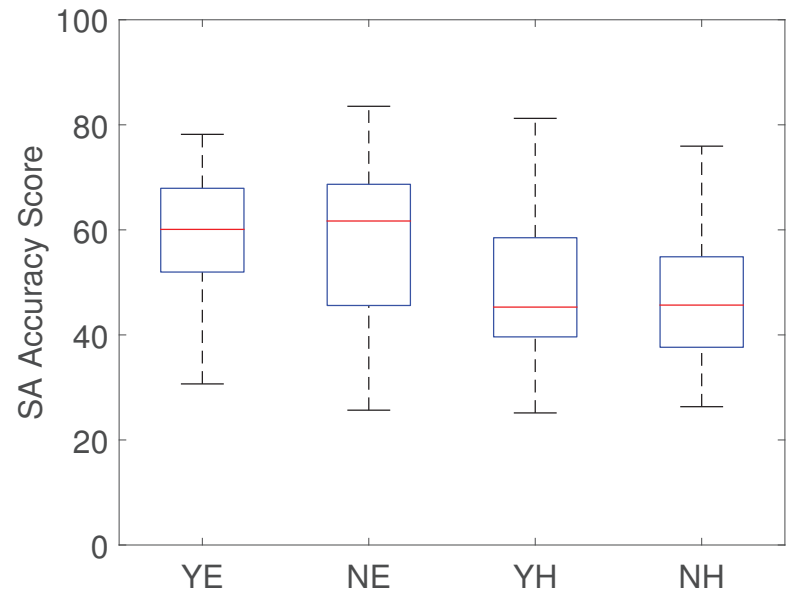

(a)

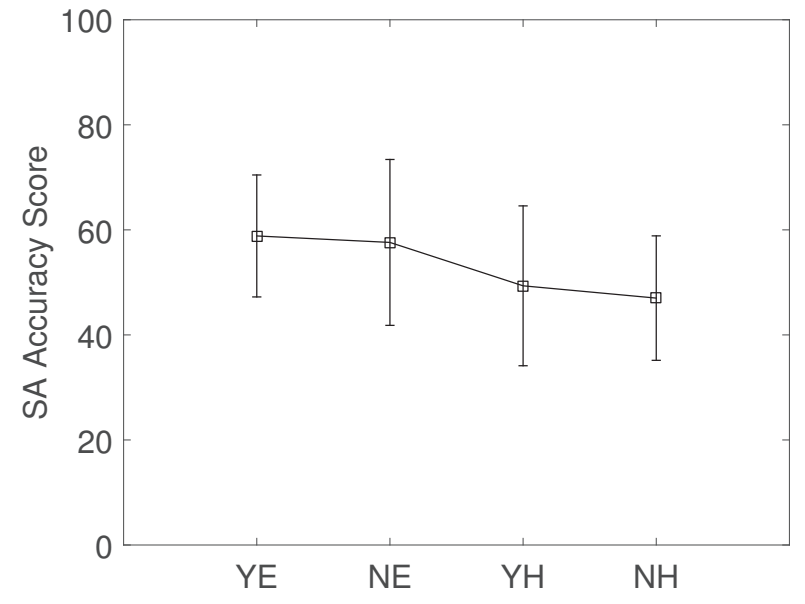

(b)

Figure 5.15: Boxplots (a) and mean/standard deviation plots (b) for SA accuracy score 
Upon further inspection of Figure 5.15b, there is a slight trend that suggests that haptic feedback may increase SA $(\mathrm{N}-\mathrm{Y}$ mean $=-2.5017)$, but is not significant enough to draw conclusions. These results do suggest, however, that haptic feedback does not have a negative effect on the operator's SA accuracy, which is also insightful.

\subsection{Results Summary}

The results are primarily focused on identifying whether or not the operator benefited from haptic feedback by evaluating measures that correspond to primary task performance (finding hotspots), secondary task performance (responding to incoming commands), SA and workload. Significant differences in world difficulty alone are used to validate easy and hard world definitions and distinguish between low and high task demand environments, respectively.

In general, haptic feedback improved the operator's ability to find hotspots in a multitasking environment that required the operator to view multiple sources of information. There was also evidence to suggest that haptic feedback decreased the manipulation time needed to find hotspots and allowed the operator to use shape manipulation more efficiently. Due to the significant benefit of haptic feedback in these areas, it can be concluded that haptic feedback does make it easier for the operator to maintain heads-up control of a team of robots. This is not to say that any presence of haptic feedback will always produce a heads-up control, but these results are hoped to be generalizable to other control modes where haptic feedback provides an advantage over visual feedback alone. On average, subjects preferred haptic feedback based on their own ratings after completing the experiment, with the exception of feedback in the travel mode, which was rated almost equally between preferred and not preferred.

Response time to incoming commands, whether team manipulation commands or typed responses to SA questions, was not affected significantly by haptic feedback. There was a trend favoring haptic feedback in SA response time that was considered marginally significant and is noticeable in plots of the response times. Overall, it is reasonable to conclude that haptic feedback has no large effect on response time, because the feedback was not designed to directly influence response time. The results do show, however, that haptic feedback did not negatively affect response time and should be included or excluded based on preference in this regard. 
The results show that haptic feedback had neither a significant positive or negative effect on SA score as a whole. Both SA response time and SA accuracy score showed improvements with haptic feedback, but those results were not significant. Because this specific SA measurement technique (involving types of SA questions and division of accuracy and response time scores) has not been tested previously, the results also suggest validity to this method. World difficulty did have a significant effect on SA Score and the results show that SA increases as task demand is lower, which is reasonable.

Workload scores were not significant but do suggest that haptic feedback might be a source of higher workload on the operator. Due to the subjectivity of the NASA TLX measure, it is difficult to determine how accurate this is without further testing. Overall, workload increased significantly when the operator managed the team in environments with higher task demand. Although haptic feedback was not shown to decrease operator workload, significant increases in other performance measures may likely be even more useful.

\subsection{Chapter Summary}

This chapter presented and discussed the results from the statistical analysis on data collected from the human subject experiment. Effects of haptic feedback and world difficulty were explained for several measures and conclusions were made on an operator's ability to control a team of robots using haptic feedback. The summary of these results was given in Section 5.3. Chapter 6 will conclude this thesis and present areas for future work. 


\section{CHAPTER 6. CONCLUSION}

This thesis has presented a global, shape-based interaction method of controlling a small team of robots by a human operator using haptic feedback. The purpose of the shape-based haptic interaction is to improve an operator's ability to perform patrol and cordon operations and help to maintain heads-up awareness. An experimental simulator was developed to create a generic multitasking environment where the operator was asked to complete reconnaissance and surveillance tasks using a team of robots. In a human subject experiment, task performance, situation awareness and workload were compared with and without haptic feedback in tasks of varying levels of difficulty. This chapter details contributions made by this research, summarizes results from the experiment, and presents areas of future work.

\subsection{Contributions}

Contributions of this work include a novel approach to shape-based robot team interaction, novel haptic feedback mapped to team modes, and the development of an experimental simulation test bed. These contributions are discussed in the sections that follow.

\subsubsection{Shape-based Robot Team Interaction}

Few swarm control methods have enabled a human operator to manage the swarm by creating custom formations. This thesis presents a novel approach that involves treating the team as a moldable volume, where deformations of the volume correspond to changes in team shape. The operator manipulates this shape while a cordon is formed around a building, or when the robot team has completely surrounded the building of choice. This restricts shape deformations that the operator can apply to a single-ring topology. This limitation is reasonable due to the desire of maintaining a cordon around the building in the proposed patrol and cordon scenario. In addition to manipulating the surrounding shape of the team, the operator also adjusts how the robots are 
spaced relative to one another by specifying regions of high robot density within the shape. This allows the operator to quickly increase the number of robots in a region of interest and maximize reconnoitering efforts in that area.

While in patrol, or travel, mode, the operator views, but does not control, the overall shape of the team, represented by the outer boundary of a geometric convex hull of robot positions. Operator control is minimal while the team is traveling between buildings to allow an increased attention to be placed on additional tasks. In other words, once a travel location is chosen, the operator may rely on the autonomous behavior of the team to travel to and surround a building of choice in order to focus attention elsewhere on other important tasks.

\subsubsection{Novel Haptic Feedback Mapped to Team Modes}

Haptic feedback was developed for each mode of team interaction. During surround mode, haptic feedback allows the operator to manipulate and feel the surrogate shape based on movements by the haptic cursor on screen. The magnitude and direction of this feedback is proportional to how the operator uses the haptic cursor to penetrate a nodal network of mass-spring-damper nodes. This feedback is used in both exploring team shape and manipulating it. Thus, the operator feels a virtual force similar to that of manipulating modeling clay on a surface. Similar resistive forces due to pulling motions are incorporated into the feedback algorithm designed for spacing manipulation. In addition to visually identifying how robots are forming within the shape, haptic feedback provides a simple way for the operator to receive similar information without the need for heads-down attention to the visual display.

During travel mode, there are two types of feedback. One type helps the operator sense the relative distance between the cursor and the team in order to gain a sense for how and where the team is traveling. The force becomes greater as the relative distance increases. The other type allows the operator to explore the overall shape of the team, enabling the interpretation of team shape and scale as it travels from building to building. The shape is modeled as an enclosed deformable volume, where the nodal network spans across the convex hull boundary and a virtual surface lies tangent to the top of the nodes. 
In either mode, a ground feedback force is produced when the haptic cursor comes in contact with the ground surface. This force helps to identify the plane in which the team motion occurs.

\subsubsection{Experimental Simulation Test Bed}

An experimental simulation test bed was developed to evaluate an operator's effectiveness in heads-up management of a small team of robots while performing reconnaissance and surveillance tasks. The simulator was specifically designed to require the operator to view multiple displays to succeed in the given tasks. One monitor displays the environment, haptic cursor, and robot team. The other monitor displays incoming messages and sensor readings from each robot. This setup simulates a scenario in which an operator is encouraged to manage the team on one display and still be aware of other information from another source, a situation which heads-up control of the team would make it easier for the operator to multitask. In contrast, prolonged focus on the team display (heads-down) would likely cause the operator to miss pertinent or time-sensitive information.

\subsection{Experimental Results}

The human subject experiment resulted in several significant results. In the experiment, the operator's effectiveness in managing the team was measured using levels of performance, SA and workload.

Performance measures were primarily concerned with the operator's ability to use the team's sensor readings to uncover hidden hotspots in the environment. Overall, the results suggest that haptic feedback significantly improves the operator's ability to find hotspots when under higher task demand. Shape manipulation by the operator was also more time efficient when using haptic feedback. This could be partly due to how the haptic channel is used to relieve the operator of excessive visual stimulation. Operators may also feel more comfortable or capable with shape-based interaction, regardless of other tasks, when haptic feedback is present. There were no significant benefits to using haptic feedback during spacing manipulation or travel mode. Haptic feedback likely made no difference in travel mode due to the simplicity of the travel objective. 
Ultimately, these results show that under a higher task demand, haptic feedback made it easier for the operator to maintain heads-up control and achieve a greater level of performance as defined by this specific experimental simulation. It is not presumed that this will always be the case in any haptic-based interface used to navigate a small team of robots, but it is hoped that these results will be useful in the development of future interfaces.

In responding to either team manipulation commands or SA questions, haptic feedback had no significant effect on response time. Although there were some improvements in SA score with haptic feedback, they were not significant. The only significant differences to SA score were due to world difficulty level, which is meaningful because it suggests that the chosen SA measurement technique did produce reasonable SA scores that follow a similar trend of operator SA.

It was hoped that haptic feedback would reduce operator workload. The results show, however, that haptic feedback might increase it, even if the results were not significant. Subjective ratings such as the NASA TLX are always questionable, but it is possible that haptic feedback may be a source of higher workload. Even if operator workload has increased, it may still be worthwhile since operator performance has increased during the process.

\subsection{Future Work}

This thesis has investigated how the use of haptic feedback can benefit a single operator in managing a small team of robots while performing other tasks. Additional work is required to verify these results and gain further insight into the design of multimodal interfaces that benefit the operator. Future work could include:

- Adjust haptic feedback parameters. Some operators prefer a minimal amount of feedback and some prefer a great deal of feedback. The feedback parameters were tuned based on comments from operators in initial trial runs and the pilot study, but miniature studies could be done to find optimal feedback magnitudes for each interaction mode. Methods to automatically tune these parameters to fit operator preference may also be desirable.

- Improve haptic cursor workspace. Based on comments from subjects in the experiment, the existence of a three-dimensional haptic cursor workspace caused initial confusion due to the robot team only traveling within a single plane. This caused much of the out-of-plane 
workspace to be unused and any out-of-plane feedback became non-intuitive to the operator. The workspace could be used more strategically in future work. For example, the ground surface could house the movement of ground vehicles and an "aerial" surface could house the movement of aerial vehicles. This would potentially allow an operator to control and manipulate two teams of robots by simply switching in and out of plane. Similar interaction methods to those proposed in this work could be easily used for teams on separate surfaces. Either something of this sort could be implemented or the haptic cursor should be simply restricted to two dimensions. This would not affect the current haptic feedback algorithms too greatly.

- Improve team interaction methods. Shape manipulation methods could be adjusted to allow for shape attraction to the haptic cursor as well as repulsion. This may allow the operator to manipulate the team shape more precisely without overshooting. Some operators found it confusing when the spacing of the entire team was adjusted during each manipulation. A more localized approach could be developed as a separate option or method to improve the intuitiveness of spacing manipulation. Other control modes can also be investigated, although development of additional modes introduces the danger of mode confusion for operators.

- Improve haptic feedback algorithms. The current algorithms focus on providing shape and spacing team information to the operator. Additional haptic cues could be provided to signal when incoming messages arrive or how strong sensor readings are in a given area. This could be provided in the form of vibrotactile feedback. If designed appropriately, the operator will have a heightened awareness of these events and not need to rely solely on visual information.

- Improve realism of team environments. RoTHSim was designed to investigate how haptic feedback can augment visual feedback. Thus, to avoid visual stimuli from being too great with the already intensive tasks that were required of the operator, the environment appearance was kept relatively simple. Additional development could be done to create an environment that would appear similar to a real-world environment. The level of detail given 
to the environment may or may not influence an operator's performance, and also may be worth investigating. 


\section{REFERENCES}

[1] Goodrich, M. A., Morse, B. S., Gerhardt, D., Cooper, J. L., Quigley, M., Adams, J. A., and Humphrey, C., 2008. "Supporting wilderness search and rescue using a camera-equipped mini UAV." Journal of Field Robotics, 25(1-2), pp. 89-110. ii, 1

[2] Kumar, V., Rus, D., and Singh, S., 2004. "Robot and sensor networks for first responders." Pervasive Computing, IEEE, 3(4), Oct, pp. 24-33. ii, 1

[3] Alboul, L., Saez-Pons, J., and Penders, J., 2008. "Mixed human-robot team navigation in the GUARDIANS project." In Proccedings of the International Conference on Safety, Security, and Rescue Robotics. ii, 1

[4] Jones, H., and Hinds, P., 2002. "Extreme work teams: Using SWAT teams as a model for coordinating distributed robots." In Proceedings of the 2002 ACM Conference on Computer Supported Cooperative Work, CSCW '02, ACM, pp. 372-381. ii, 1

[5] Jung, S., and Goodrich, M. A., 2013. "Shaping couzin-like torus swarms through coordinated mediation." In Systems, Man, and Cybernetics (SMC), 2013 IEEE International Conference on, IEEE, pp. 1834-1839. ii, 1

[6] Maxwell, P., Maciejewski, A. A., Siegel, H. J., Potter, J., and Smith, J. E., 2009. "A mathematical model of robust military village searches for decision making purposes.." In IKE, pp. 311-316. ii, 1

[7] Murphy, R. R., and Burke, J. L., 2010. "The safe human-robot ratio." In Human-Robot Interaction in Future Military Operations, M. Barnes and F. Jentsch, eds. Ashgate Publishing, ch. 3, pp. 31-49. ii, 1

[8] Lewis, M., 2013. "Human Interaction With Multiple Remote Robots." Reviews of Human Factors and Ergonomics, 9, pp. 131-174. ii, 1

[9] Kolling, A., Walker, P., Member, S., Chakraborty, N., Sycara, K., and Lewis, M., 2015. "Human Interaction With Robot Swarms : A Survey." pp. 1-18. ii, 1

[10] Goodrich, M. A., 2010. "On maximizing fan-out: Towards controlling multiple unmanned vehicles." In Human-Robot Interactions in Future Military Operations, M. Barnes and F. Jentsch, eds. Ashgate Publishing, Surrey, England. 1

[11] Whetten, J. M., Goodrich, M. A., and Guo, Y., 2010. "Beyond robot fan-out: Towards multioperator supervisory control." In Proceedings of IEEE International Conference on Systems, Man, and Cybernetics. 1

[12] Wickens, C. D., 2002. "Multiple resources and performance prediction." Theoretical Issues in Ergonomics Science, 3(2), pp. 159-177. 2 
[13] Brandt, A. M., and Colton, M. B., 2010. "Haptic collision avoidance for a remotely operated quadrotor UAV in indoor environments." In Proc. IEEE Int Systems Man and Cybernetics (SMC) Conf, pp. 2724-2731. 2, 3

[14] Philbrick, R. M., and Colton, M. B., 2014. "Effects of Haptic and 3D Audio Feedback on Operator Performance and Workload for Quadrotor UAVs in Indoor Environments." Journal of Robotics and Mechatronics, 26(5), pp. 580-591. 2, 3, 8

[15] Nunnally, S., Walker, P., Lewis, M., Chakraborty, N., and Sycara, K., 2013. "Using Haptic Feedback in Human Robotic Swarms Interaction." Proceedings of the Human Factors and Ergonomics Society Annual Meeting, 57(1), sep, pp. 1047-1051. 2, 3

[16] Nunnally, S., Walker, P., Chakraborty, N., Lewis, M., and Sycara, K., 2013. "Using Coverage for Measuring the Effect of Haptic Feedback in Human Robotic Swarm Interaction." 2013 IEEE International Conference on Systems, Man, and Cybernetics, oct, pp. 516-521. 2, 33

[17] Setter, T., Kawashima, H., and Egerstedt, M., 2015. "Team-Level Properties for Haptic Human-Swarm Interactions *." American Control Conference. 2, 4

[18] Lam, T. M., Boschloo, H. W., Mulder, M., van Paassen, M. M., and van der Helm, F. C. T., 2004. "Effect of haptic feedback in a trajectory following task with an unmanned aerial vehicle." In Proc. IEEE Int Systems, Man and Cybernetics Conf, Vol. 3, pp. 2500-2506. 2, 3

[19] Lam, T. M., Mulder, M., and van Paassen, M. M., 2009. "Haptic interface in uav teleoperation using force-stiffness feedback." In Proc. IEEE Int. Conf. Systems, Man and Cybernetics SMC 2009, pp. 835-840. 2

[20] Sumpter, D. J. T., 2012. Collective Animal Behavior. Princeton University Press, Princeton, N.J. 2

[21] Goodrich, M. a., Pendleton, B., Sujit, P. B., and Pinto, J., 2011. "Toward human interaction with bio-inspired robot teams." 2011 IEEE International Conference on Systems, Man, and Cybernetics, oct, pp. 2859-2864. 2

[22] Bayindir, L., 2016. “A review of swarm robotics tasks.” Neurocomputing, 172, pp. 292-321. 3

[23] Alder, C. K., McDonald, S. J., Colton, M. B., and Goodrich, M. A., 2015. "Toward hapticbased management of small swarms in cordon and patrol." 2015 Swarm/Human Blended Intelligence Workshop (SHBI), pp. 1-8. 3,8

[24] Lam, TM; Mulder, M.; van Paassen, M., 2009. "Haptic interface in UAV tele-operation using force-stiffness feedback.” In Proc. IEEE Int. Conf. Systems, Man and Cybernetics(October), pp. $835-840.3$

[25] Philbrick, R. M., and Colton, M. B., 2014. "Effects of haptic and 3D audio feedback on operator performance and workload for quadrotor UAVs in indoor environments." Journal of Robotics and Mechatronics, 26(5), pp. 580-591. 3, 6 
[26] Fields, M. A., Haas, E., Hill, S., Stachowiak, C., and Barnes, L., 2009. "Effective robot team control methodologies for battlefield applications." 2009 IEEE/RSJ International Conference on Intelligent Robots and Systems, IROS 2009, pp. 5862-5867. 4, 31

[27] Franchi, A., Robuffo Giordano, P., Secchi, C., Son, H. I., and Bulthoff, H. H., 2011. "A passivity-based decentralized approach for the bilateral teleoperation of a group of UAVs with switching topology." 2011 IEEE International Conference on Robotics and Automation, may, pp. 898-905. 4

[28] Chen, J. Y. C., and Barnes, M. J., 2014. "HumanAgent Teaming for Multirobot Control: A Review of Human Factors Issues." IEEE Transactions on Human-Machine Systems, 44(1), pp. 13-29. 4, 31

[29] Endsley, M. R., 1988. "Situation awareness global assessment technique (SAGAT)." Aerospace and Electronics Conference, 1988. NAECON 1988., Proceedings of the IEEE 1988 National, pp. 789-795. 4, 5, 37

[30] Flach, J. M., 1995. "Situation Awareness: Proceed with Caution." Human Factors, 37(1), pp. 149-157. 4

[31] Flach, J. M., 2015. "Situation Awareness: Context Matters! A Commentary on Endsley ." Journal of Cognitive Engineering and Decision Making, 9(1), pp. 59-72. 4

[32] Hone, G. N., Martin, L., and Ayres, R., 2006. "Awareness does the acronym "SA" still have any value?." 11th International Command and Control Research and Technology Symposium, 44(0). 4, 35

[33] Taylor, R. M., 1990. "Situational awareness rating technique (SART): The development of a tool for aircrew systems design." Situational Awareness in Aerospace Operations AGARDCP-478), pp. 3/1-3/17. 5

[34] Endsley, M. R., Selcon, S. J., Hardiman, T. D., and Croft, D. G., 1998. "A comparative analysis of SAGAT and SART for evaluations of situation awareness." Proceedings of the Human Factors and Ergonomics Society Annual Meeting, 42(1), pp. 82-86. 5

[35] Chen, J. Y. C., and Barnes, M. J., 2015. "Agent Transparency for Human-Agent Teaming Effectiveness." pp. 1381-1385. 5

[36] Garland, D. J., Endsley, M. R., Andre, A. D., Hancock, P. a., Selcon, S. J., and Vidulich, M. a., 1996. "Assessment and measurement of situation awareness." pp. 1170-1173. 5

[37] Hancock, P. A., and Meshkati, N., 1988. Human Mental Workload. Elsevier Science Publishers. 5

[38] Novak, D., Mihelj, M., and Munih, M., 2011. "Psychophysiological responses to different levels of cognitive and physical workload in haptic interaction." Robotica, 29(03), pp. 367374. 5,6

[39] Nakayama, O., Futami, T., Nakamura, T., and Boer, E. R., 1999. Development of a steering entropy method for evaluating driver workload. No. 1999-01-0892. Society of Automotive Engineers, pp. -01-0892. 6 
[40] Hart, S. G., and Staveland, L. E., 1988. "Development of NASA-TLX (Task Load Index): Results of Empirical and Theoretical Research." Advances in Psychology, 52(C), pp. 139183. 6

[41] Diana, M., De La Croix, J. P., and Egerstedt, M., 2013. "Deformable-medium affordances for interacting with multi-robot systems." IEEE International Conference on Intelligent Robots and Systems, pp. 5252-5257. 10, 11

[42] de Berg, M., van Kreveld, M., Overmars, M., and Schwarzkopf, O., 2000. Computational Geometry: Algorithms and Applications. Springer. 21

[43] CHAI 3D, 2016. [Online] Available at http://www.chai3d.org/support.html [Accessed: 07/20/2016]. 25

[44] Google Protocol Buffers, 2016. [Online] Available at https://developers.google.com/ protocol-buffers [Accessed: 07/20/2016]. 26

[45] Barnes, M., Jentsch, F., Chen, J. Y. C., Haas, E., and Cosenzo, K., 2010. "Soldier-Robot Teams: Six Years of Research." Proceedings of the Human Factors and Ergonomics Society Annual Meeting, 54(19), sep, pp. 1493-1497. 31

[46] Chen, J. Y. C., and Terrance, P. I., 2008. "Effects of tactile cueing on concurrent performance of military and robotics tasks in a simulated multi-tasking environment." Ergonomics, 51(8), pp. 1137-1152. 31 